CRM-2852 (2002)

Saclay-T02/097

nlin.SI/0208002

\title{
Differential systems for biorthogonal polynomials appearing in 2-matrix models and the associated Riemann-Hilbert problem
}

\author{
M. Bertola $a^{\dagger \dagger}$, B. Eynard ${ }^{\dagger \star 3}$ and J. Harnad ${ }^{\dagger+}$ \\ † Centre de recherches mathématiques, Université de Montréal \\ C. P. 6128, succ. centre ville, Montréal, Québec, Canada H3C 3J7 \\ $\ddagger$ Department of Mathematics and Statistics, Concordia University \\ 7141 Sherbrooke W., Montréal, Québec, Canada H4B 1R6 \\ * Service de Physique Théorique, CEA/Saclay \\ Orme des Merisiers F-91191 Gif-sur-Yvette Cedex, FRANCE
}

\begin{abstract}
We consider biorthogonal polynomials that arise in the study of a generalization of two-matrix Hermitian models with two polynomial potentials $V_{1}(x), V_{2}(y)$ of any degree, with arbitrary complex coefficients. Finite consecutive subsequences of biorthogonal polynomials ("windows"), of lengths equal to the degrees of the potentials $V_{1}$ and $V_{2}$, satisfy systems of ODE's with polynomial coefficients as well as PDE's (deformation equations) with respect to the coefficients of the potentials and recursion relations connecting consecutive windows. A compatible sequence of fundamental systems of solutions is constructed for these equations. The (Stokes) sectorial asymptotics of these fundamental systems are derived through saddle-point integration and the Riemann-Hilbert problem characterizing the differential equations is deduced.
\end{abstract}

\footnotetext{
${ }^{1}$ Work supported in part by the Natural Sciences and Engineering Research Council of Canada (NSERC) and the Fonds FCAR du Québec.

${ }^{2}$ bertola@crm.umontreal.ca

3 eynard@spht.saclay.cea.fr

${ }^{4}$ harnad@crm.umontreal.ca
} 


\section{Introduction}

In [2, 3] the differential systems satisfied by sequences of biorthogonal polynomials associated to 2matrix models were studied, together with the deformations induced by changes in the coefficients of the potentials determining the orthogonality measure. For ensembles consisting of pairs of $N \times N$ hermitian matrices $M_{1}$ and $M_{2}$, the $U(N)$ invariant probability measure is taken to be of the form:

$$
\frac{1}{\tau_{N}} d \mu\left(M_{1}, M_{2}\right):=\frac{1}{\tau_{N}} \exp \frac{1}{\hbar} \operatorname{tr}\left(-V_{1}\left(M_{1}\right)-V_{2}\left(M_{2}\right)+M_{1} M_{2}\right) d M_{1} d M_{2} .
$$

where $d M_{1} d M_{2}$ is the standard Lebesgue measure for pairs of Hermitian matrices and the potentials $V_{1}$ and $V_{2}$ are chosen to be polynomials of degrees $d_{1}+1, d_{2}+1$ respectively, with real coefficients. The overall positive small parameter $\hbar$ in the exponential is taken of order $N^{-1}$ when considering the large $N$ limit, but in the present context it will just play the role of Planck's constant in the string equation.

Using the Harish-Chandra-Itzykson-Zuber formula, one can reduce the computation of the corresponding partition function to an integral over only the eigenvalues of the two matrices

$$
\tau_{N}:=\iint d \mu\left(M_{1}, M_{2}\right) \propto \int \prod_{i=1}^{N} \mathrm{~d} x_{i} \mathrm{~d} y_{i} \Delta(x) \Delta(y) \mathrm{e}^{-\frac{1}{\hbar} \sum_{j=1}^{N} V_{1}\left(x_{j}\right)+V_{2}\left(y_{j}\right)-x_{j} y_{j}},
$$

and then express all spectral statistics in terms of the associated biorthogonal polynomials, in the same spirit as orthogonal polynomials are used in the spectral statistics of one-matrix models [24]. In this context, what is meant by biorthogonal polynomials is a pair of sequences of monic polynomials

$$
\pi_{n}(x)=x^{n}+\cdots, \quad \sigma_{n}(y)=y^{n}+\cdots, \quad n \in \mathbb{N}
$$

which are mutually dual with respect to the associated coupled measure on the product space

$$
\int_{\mathbb{R}} \int_{\mathbb{R}} \mathrm{d} x \mathrm{~d} y \pi_{n}(x) \sigma_{m}(y) \mathrm{e}^{-\frac{1}{\hbar}\left(V_{1}(x)+V_{2}(y)-x y\right)}=h_{n} \delta_{m n} .
$$

In this work, we use essentially the same definition of orthogonality, but extend it to the case of polynomials $V_{1}$ and $V_{2}$ with arbitrary (possibly complex) coefficients, with the contours of integration no longer restricted to the real axis, but taken as curves in the complex plane starting and ending at $\infty$, chosen so that the integrals are convergent. The orthogonality relations determine the two families uniquely, if they exist 13, 33.

It was shown in 2, 3] that the finite consecutive subsequences of lengths $d_{2}+1$ and $d_{1}+1$ respectively, within the sequences of dual quasi-polynomials:

$$
\psi_{n}(x)=\frac{1}{\sqrt{h_{n}}} \pi_{n}(x) \mathrm{e}^{-\frac{1}{\hbar} V_{1}(x)}, \quad \phi_{n}(y)=\frac{1}{\sqrt{h_{n}}} \sigma_{n}(y) \mathrm{e}^{-\frac{1}{\hbar} V_{2}(y)},
$$

beginning (or ending) at the points $n=N$, satisfy compatible overdetermined systems of first order differential equations with polynomial coefficients of degrees $d_{1}$ and $d_{2}$, respectively, as well as recursion 
relations for consecutive values of $N$. In fact, certain quadruples of Differential-Deformation-Difference equations (DDD for short) were derived for these "windows", as well as for their Fourier Laplace transforms, in which the deformation parameters were taken to be the coefficients of the potentials $V_{1}$ and $V_{2}$. It was shown in 2, 3] that these systems are Frobenius compatible and hence admit joint fundamental systems of solutions.

In the present work we explicitly construct such fundamental systems in terms of certain integral transforms applied to the biorthogonal polynomials. The main purpose is to derive the Riemann-Hilbert problem characterizing the sectorial asymptotic behavior at $x=\infty$ or $y=\infty$.

The ultimate purpose of this analysis is to deduce in a rigorous way the double-scaling limits $N \rightarrow$ $\infty, \hbar N=\mathcal{O}(1)$ of the partition function and spectral statistics, for which the corresponding large $N$ asymptotics of the biorthogonal polynomials are required. (See 12, 22 and references therein for further background on 2-matrix models, and 19, 14, 15, 16, 13 for other more recent developments.) The study of the large $N$ limit of matrix integrals is of considerable interest in physics, since many physical systems having a large number of strongly correlated degrees of freedom (quantum chaos, mesoscopic conductors, ...) share the statistical properties of the spectra of random matrices. Also, the large $N$ expansion of a random matrix integral (if it exists) is expected to be the generating functional of discretized surfaces, and therefore random matrices provide a powerful tool for studying statistical physics on a random surface. (The 2-matrix model was first introduced in this context, as the Ising model on a random surface [22].)

It has been understood for some time that the 1-matrix model is not general enough, since it cannot represent all models of statistical physics (e.g., it contains only the $(p, 2)$ conformal minimal models). In order to recover the missing conformal models $((p, q)$ with $p$ and $q$ integers), it is necessary to introduce at least a two-matrix model [9]. The 1-matrix models are actually included in the 2-matrix models, since if one takes $d_{2}=1$, and integrates over the Gaussian matrix $M_{2}$, one sees that the 1-matrix model follows, and hence may be seen as a particular case.

It should also be mentioned that most of the results about the 2-matrix model (in particular those derived in the present work) can easily be extended to multi-matrix models without major modifications (see the appendix of [2]). Indeed, the multi-matrix model is not expected to be very different from the 2-matrix case [9]. (In particular, it contains the same conformal models.)

The present paper is organized as follows: in Section 2, we present the required formalism for biorthogonal polynomials, beginning with the systems of differential and recursion relations they satisfy, and recalling the main definitions and results of [2]. We then derive the fundamental systems of solutions to the overdetermined systems for the "windows" of biorthogonal polynomials in two ways: one, by exploiting the recursion matrices $Q, P$ for the biorthogonal polynomials, which satisfy the string equation, and another by giving explicit integral formulas for solutions and showing their independence when taken over a suitably defined homology basis of inequivalent integration paths. 
In Section 3 we use saddle-point integration methods to deduce the asymptotic form of these fundamental systems of solutions within the various Stokes sectors, and from these, to deduce the Stokes matrices and jump discontinuities at $\infty$. The full formulation of the matrix Riemann-Hilbert problem characterizing these solutions is given in Theorem 3.1.

Acknowledgements: The authors would like to thank A. Kapaev for helpful comments concerning nonsingular integral representations of the fundamental systems of solutions to eq. (2.1.38), which resulted in the addition of appendix $\mathrm{A}$ and the discussion at the end of Sec. 2.3 .

\section{Differential systems for biorthogonal polynomials and funda- mental solutions}

\subsection{Biorthogonal polynomials, recursion relations and differential systems}

The definitions and notation to be used generally follow [2], with some minor modifications, and will be recalled here. Let us fix two polynomials which will be referred to as the "potentials",

$$
V_{1}(x)=u_{0}+\sum_{K=1}^{d_{1}+1} \frac{u_{K}}{K} x^{K}, \quad V_{2}(y)=v_{0}+\sum_{J=1}^{d_{2}+1} \frac{v_{J}}{J} y^{J} .
$$

In terms of these potentials we define a bimoment functional, i.e., a bilinear pairing between polynomials $\pi(x)$ and $\sigma(y)$ by means of the following formula

$$
(\pi, \sigma):=\int_{\Gamma^{(x)}} \int_{\Gamma^{(y)}} \mathrm{d} x \mathrm{~d} y \mathrm{e}^{-\frac{1}{\hbar}\left(V_{1}(x)+V_{2}(y)-x y\right)} \pi(x) \sigma(y) .
$$

The contours of integration $\Gamma^{(x)}, \Gamma^{(y)}$ in the complex $x$ and $y$ planes remain to be specified. They will generally be chosen to begin and end at $\infty$, approaching it asymptotically in any direction that assures convergence. More generally, linear combinations of such integrals along various inequivalent contours may also be chosen. In fact there are precisely $d_{1}$ (homologically) independent choices for the individual contours in the $x$ plane and $d_{2}$ in the $y$-plane, due to the choice of the integrands [5]. The necessary and sufficient condition for the convergence of these integrals is that the contours approach $\infty$ in such a way that

$$
\Re\left(V_{1}(x)\right) \underset{x \rightarrow \infty}{\longrightarrow}+\infty, \quad \Re\left(V_{2}(y)\right) \underset{y \rightarrow \infty}{\longrightarrow}+\infty .
$$

For $k=0, \ldots 2 d_{1}+1\left(\bmod 2 d_{1}+2\right)$ and $l=0, \ldots 2 d_{2}+1\left(\bmod 2 d_{2}+2\right)$, let us define the sectors

$$
\begin{aligned}
& \mathcal{S}_{k}^{(x)}:=\left\{x \in \mathbb{C}, \arg (x) \in\left(\vartheta_{x}+\frac{(2 k-1) \pi}{2\left(d_{1}+1\right)}, \vartheta_{y}+\frac{(2 k+1) \pi}{2\left(d_{1}+1\right)}\right)\right\}, \\
& \vartheta_{x}:=-\arg \left(u_{d_{1}+1}\right) /\left(d_{1}+1\right) \text {, } \\
& \mathcal{S}_{l}^{(y)}:=\left\{y \in \mathbb{C}, \arg (y) \in\left(\vartheta_{y}+\frac{(2 l-1) \pi}{2\left(d_{2}+1\right)}, \vartheta_{y}+\frac{(2 l+1) \pi}{2\left(d_{2}+1\right)}\right)\right\}, \\
& \vartheta_{y}:=-\arg \left(v_{d_{2}+1}\right) /\left(d_{2}+1\right) \text {. }
\end{aligned}
$$


Definition 2.1 (See Fig. 1) The wedge contours in the x-plane (resp. y-plane) $\Gamma_{i}^{(x)}$ (resp. $\Gamma_{j}^{(y)}$ ) for $i=0, \ldots d_{1}$ (resp. $j=0, \ldots d_{2}$ ), and the anti-wedge contours $\tilde{\Gamma}_{i}^{(x)}$ (resp. $\tilde{\Gamma}_{j}^{(y)}$ ) for $i=0, \ldots d_{1}$ (resp. $\left.j=0, \ldots d_{2}\right)$ are defined as follows:

- $\Gamma_{i}^{(x)}$ (resp. $\Gamma_{j}^{(y)}$ ) comes from $\infty$ within the sector $\mathcal{S}_{2 i-2}^{(x)}$ (resp. $\mathcal{S}_{2 j-2}^{(y)}$ ) and returns to infinity in the sector $\mathcal{S}_{2 i}^{(x)}\left(\right.$ resp. $\left.\mathcal{S}_{2 j}^{(y)}\right)$.

- $\tilde{\Gamma}_{i}^{(x)}$ (resp. $\tilde{\Gamma}_{j}^{(y)}$ ) comes from $\infty$ within the sector $\mathcal{S}_{2 i-1}^{(x)}$ (resp. $\mathcal{S}_{2 j-1}^{(y)}$ ) and returns to infinity in the sector $\mathcal{S}_{2 i+1}^{(x)}\left(\right.$ resp. $\left.\mathcal{S}_{2 j+1}^{(y)}\right)$.

They have the property that $\Re V_{1}(x) \rightarrow \infty$ on the wedge contours $\Gamma_{i}^{(x)}$ and $\Re V_{1}(x) \rightarrow-\infty$ on the antiwedge contours $\widetilde{\Gamma}_{i}^{(x)}$ as $|x| \rightarrow \infty$ (and similarly for $V_{2}(y)$ ).

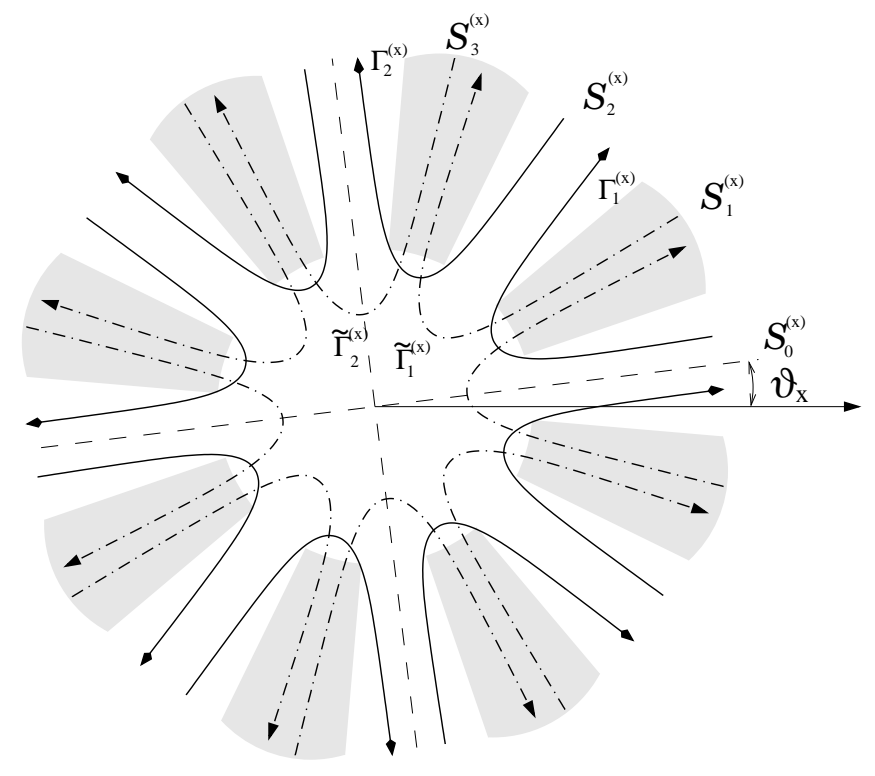

Figure 1: An example of wedge (solid) and anti-wedge (line-dot-line) contours in the $x$ plane for a case with $d_{1}=7$ and real positive leading coefficient in $V_{1}$. The white sectors are the even numbered ones and the gray sectors the odd-numbered ones. (Only the first few are labelled).

Note that since there are no singularities in the finite region of the $x$ - or $y$-planes for the integrands we are considering, we can deform the contours $\Gamma_{j}^{(x)}$ and $\Gamma_{k}^{(y)}$ arbitrarily in the finite part of the $x$ and $y$ planes. We could therefore also take such contours as straight rays coming from the $(2 k-2)$-th sector combined with another ray going to infinity within the $2 k$-th sector. Moreover, by the Cauchy theorem, we have the homological relation

$$
\sum_{i=0}^{d_{1}} \Gamma_{i}^{(x)} \equiv 0, \quad \sum_{j=0}^{d_{2}} \Gamma_{j}^{(y)} \equiv 0, \text { homologically } .
$$


There are therefore are only $d_{2}$ homologically independent contours $\Gamma_{k}^{(y)}$ and $d_{1}$ of type $\Gamma_{k}^{(x)}$. (Similar remarks apply to the anti-wedge contours which will be needed later in this article.)

In general, for any complex $d_{1} \times d_{2}$ matrix with elements $\left\{\varkappa^{i, j}\right\}_{\substack{i=1, \ldots d_{1} \\ j=1, \ldots d_{2}}}$ define the homology class of contours $\varkappa \Gamma$ to be

$$
\varkappa \Gamma:=\sum_{i j} \varkappa^{i, j} \Gamma_{i}^{(x)} \times \Gamma_{j}^{(y)}
$$

For brevity, we will denote the corresponding integral operator as follows.

$$
\sum_{i=1}^{d_{1}} \sum_{j=1}^{d_{2}} \varkappa^{i, j} \int_{\Gamma_{i}^{(x)} \times \Gamma_{j}^{(y)}}:=\int_{\varkappa \Gamma} .
$$

For a given $\varkappa$, we denote the corresponding bilinear pairing as:

$$
(\pi, \sigma)_{\varkappa}:=\int_{\varkappa \Gamma} \mathrm{d} x \mathrm{~d} y \pi(x) \mathrm{e}^{-\frac{1}{\hbar}\left(V_{1}(x)+V_{2}(y)-x y\right)} \sigma(y),
$$

and define two sequences of monic polynomials $\pi_{n}(x), \sigma_{n}(y)$ of degree $n$ such that they are biorthogonal with respect to this pairing

$$
\begin{aligned}
& \left(\pi_{n}, \sigma_{m}\right)_{\varkappa}=h_{n} \delta_{n m}, \\
& \pi_{n}(x)=x^{n}+\ldots, \sigma_{n}(y)=y^{n}+\ldots,
\end{aligned}
$$

The matrix $\varkappa^{i, j}$ must be chosen such that the $N \times N$ finite submatrices of the matrix of bimoments

$$
\left\{B_{i j}:=\left(x^{i}, y^{j}\right)\right\}_{i, j=0, \ldots, N-1}
$$

are nonsingular for all $N$; i.e., the nondegeneracy condition that ensures the existence of the biorthogonal polynomials is given by

$$
\Delta_{N}(\varkappa):=\operatorname{det}\left[\left(x^{i}, y^{j}\right)_{\varkappa}\right]_{i, j=0, \ldots, N} \neq 0 \quad \forall N \in \mathbb{N} .
$$

Since these $\Delta_{N}(\varkappa)$ 's form a denumerable sequence of homogeneous polynomials in the coefficients $\varkappa^{i, j}$, this will hold on the complement of a denumerable set of hypersurfaces, and in this sense the permissible choices of $\varkappa$ 's are "generic", whatever the choice of potentials $V_{1}$ and $V_{2}$.

We introduce the corresponding normalized quasipolynomials and combine them into semi-infinite column vectors ("wave-vectors") $\underset{\infty}{\Psi}(x), \underset{\infty}{\Phi}(y)$ as follows.

$$
\begin{aligned}
& \psi_{n}(x):=\frac{1}{\sqrt{h_{n}}} \pi_{n}(x) \mathrm{e}^{-\frac{1}{\hbar} V_{1}(x)}, \quad \phi_{n}(y):=\frac{1}{\sqrt{h_{n}}} \sigma_{n}(y) \mathrm{e}^{-\frac{1}{\hbar} V_{2}(y)}, \\
& \underset{\infty}{\Psi}(x):=\left[\psi_{0}(x), \ldots, \psi_{n}(x), \ldots\right]^{t}, \quad \underset{\infty}{\Phi}(y):=\left[\phi_{0}(y), \ldots, \phi_{n}(y), \ldots\right]^{t} .
\end{aligned}
$$


In matrix notation the biorthogonality reads

$$
\int_{\varkappa \Gamma} \mathrm{d} x \mathrm{~d} y \mathrm{e}^{\frac{x y}{\hbar}} \underset{\infty}{\Psi}(x) \underset{\infty}{\Phi^{t}}(y)=\mathbf{1},
$$

where 1 denotes the semi-infinite unit matrix.

We denote the Fourier-Laplace transforms of the wave-vectors along the wedge contours by

$$
\begin{gathered}
\underline{\Psi}_{\infty}^{(i)}(y):=\int_{\Gamma_{i}^{(x)}} \mathrm{d} x \mathrm{e}^{\frac{x y}{\hbar}} \Psi_{\infty}^{t}(x), \quad i=0, \ldots, d_{1} \\
\underline{\Phi}^{(j)}(x):=\int_{\Gamma_{j}^{(y)}} \mathrm{d} y \mathrm{e}^{\frac{x y}{\hbar}} \Phi_{\infty}^{t}(y), \quad j=0, \ldots, d_{2},
\end{gathered}
$$

viewed as row vectors, with components denoted $\underline{\psi}_{n}^{(i)}(y)$ and $\underline{\phi}_{n}^{(j)}(x)$ respectively. Because of eq. 2.1.6 only $d_{1}\left(\right.$ or $\left.d_{2}\right)$ are linearly independent

$$
\sum_{i=0}^{d_{1}} \underline{\Psi}^{(i)}(y)=\sum_{j=0}^{d_{2}} \frac{\Phi}{\infty}^{(j)}(x) \equiv 0 .
$$

The recursion relations for the biorthogonal polynomials are expressed by the following matrix equations for the wave-vectors [2]:

$$
\begin{array}{ll}
x \underset{\infty}{\Psi}(x)=Q \underset{\infty}{\Psi}(x), & \hbar \partial_{x} \underset{\infty}{\Psi}(x)=-P \underset{\infty}{\Psi}(x), \\
y \underset{\infty}{\Phi}(y)=P^{t} \underset{\infty}{\Phi}(y), & \hbar \partial_{y} \underset{\infty}{\Phi}(y)=-Q^{t} \underset{\infty}{\Phi}(y),
\end{array}
$$

where the matrices $Q$ and $P$ have finite band sizes $d_{2}+1$ and $d_{1}+1$ respectively, with $Q$ having only one nonzero diagonal above the principal one and $P$ only one below

$$
\begin{aligned}
Q & :=\left[\begin{array}{ccccc}
\alpha_{0}(0) & \gamma(0) & 0 & 0 & \cdots \\
\alpha_{1}(1) & \alpha_{0}(1) & \gamma(1) & 0 & \cdots \\
\vdots & \ddots & \ddots & \ddots & \ddots \\
\alpha_{d_{2}}\left(d_{2}\right) & \alpha_{d_{2}-1}\left(d_{2}\right) & \cdots & \alpha_{0}\left(d_{2}\right) & \gamma\left(d_{2}\right) \\
0 & \ddots & \ddots & \ddots & \ddots
\end{array}\right] \\
P & :=\left[\begin{array}{ccccc}
\beta_{0}(0) & \beta_{1}(1) & \cdots & \beta_{d_{1}}\left(d_{1}\right) & \cdots \\
\gamma(0) & \beta_{0}(1) & \beta_{1}(2) & \ddots & \beta_{d_{1}}\left(d_{1}+1\right) \\
0 & \gamma(1) & \beta_{0}(2) & \ddots & \ddots \\
0 & 0 & \gamma(2) & \beta_{0}(3) & \ddots \\
\vdots & \ddots & \ddots & \ddots & \ddots
\end{array}\right] .
\end{aligned}
$$

These semi-infinite matrices satisfy the string equation

$$
[P, Q]=\hbar \mathbf{1} .
$$


(See [2, 12, 14] for simple proofs of these assertions.) For the dual sequences of Fourier-Laplace transforms, a simple integration by parts in eqs. (2.1.19), (2.1.20) gives

$$
\begin{aligned}
& x \frac{\Phi}{\infty}^{(j)}(x)=\frac{\Phi}{\infty}^{(j)}(x) Q, \quad \hbar \partial_{x} \frac{\Phi}{\infty}^{(j)}(x)=\underline{\Phi}_{\infty}^{(j)}(x) P, \quad j=0, \ldots, d_{2} \\
& y \underline{\Psi}_{\infty}^{(k)}(y)=\underline{\Psi}_{\infty}^{(k)}(y) P^{t}, \quad \hbar \partial_{y} \frac{\Psi}{\infty}^{(k)}(y)=\underline{\Psi}_{\infty}^{(k)}(y) Q^{t}, \quad k=0, \ldots, d_{1} .
\end{aligned}
$$

Notice that integration by parts is allowed due to the exponential decay of the integrand along the chosen contours.

We recall from [2] the definition of dual sequences of windows.

Definition 2.2 Call a window of size $\mathbf{d}_{\mathbf{1}}+\mathbf{1}$ or $\mathbf{d}_{\mathbf{2}}+\mathbf{1}$ any subset of $d_{1}+1$ or $d_{2}+1$ consecutive elements of type $\psi_{n}, \underline{\phi}_{n}$, $\phi_{n}$ or $\underline{\psi}_{n}$, with the notations

$$
\begin{aligned}
& \underset{N}{\Psi}:=\left[\psi_{N-d_{2}}, \ldots, \psi_{N}\right]^{t}, \quad N \geq d_{2}, \quad \underset{N}{\Phi}:=\left[\phi_{N-d_{1}}, \ldots, \phi_{N}\right]^{t}, \quad N \geq d_{1} \\
& \stackrel{N}{\Psi}:=\left[\psi_{N-1}, \ldots, \psi_{N+d_{1}-1}\right]^{t}, \quad N \geq 0, \quad \stackrel{N}{\Phi}:=\left[\phi_{N-1}, \ldots, \phi_{N+d_{2}-1}\right]^{t}, \quad N \geq 1 \\
& \underline{\Psi}_{N}^{(j)}:=\left[\underline{\psi}_{N-d_{2}}^{(j)}, \ldots, \underline{\psi}_{N}^{(j)}\right] \quad N \geq d_{2}, \quad \underline{\Phi}_{N}^{(k)}:=\left[\underline{\phi}_{N-d_{1}}^{(k)}, \ldots, \underline{\phi}_{N}^{(k)}\right], \quad N \geq d_{1} \\
& \underline{\Psi}^{(j)}:=\left[\underline{\psi}_{N-1}^{(j)}, \ldots, \underline{\psi}_{N+d_{1}-1}^{(j)}\right], \quad N \geq 1, \quad \underline{\Phi}^{(k)}:=\left[\underline{\phi}_{N-1}^{(k)}, \ldots, \underline{\phi}_{N+d_{2}-1}^{(k)}\right], \quad N \geq 1 .
\end{aligned}
$$

Note the difference in positioning and size of the windows in the various cases, and the fact that the barred quantities are defined as row vectors while the unbarred ones are column vectors. The notation we are using here differs slightly from that used in [2], in which, e.g., $\stackrel{N}{\Phi}$ here would be denoted $\stackrel{N+1}{\Phi}$ in $[2]$.

Definition 2.3 Each of the two pairs of windows $(\underset{N}{\Psi}, \stackrel{N}{\Phi})$ and $(\underset{N}{\Phi}, \stackrel{N}{\underline{\Psi}})$ of dimensions $d_{2}+1$ and $d_{1}+1$ respectively, will be called dual windows (and these are defined for $N \geq d_{2}$ and $N \geq d_{1}$ respectively).

We now recall the notion of folding, as defined in [2]. We give here only the main statements without proofs and refer the reader to ref. [2] for further details. Let us introduce the sequence of companion-like matrices $\underset{N}{\mathbf{a}}(x)$ and $\underline{\mathbf{a}}(x)$ of size $d_{2}+1$

$$
\begin{aligned}
& \underset{N}{\mathbf{a}}(x):=\left[\begin{array}{cccc}
0 & 1 & 0 & 0 \\
0 & 0 & \ddots & 0 \\
0 & 0 & 0 & 1 \\
\frac{-\alpha_{d_{2}(N)}}{\gamma(N)} & \cdots & \frac{-\alpha_{1}(N)}{\gamma(N)} \frac{\left(x-\alpha_{0}(N)\right)}{\gamma(N)}
\end{array}\right], \quad N \geq d_{2}, \\
& \underset{N}{N}(x):=\left[\begin{array}{cccc}
\frac{x-\alpha_{0}(N)}{\gamma(N-1)} & 1 & 0 & 0 \\
\frac{-\alpha_{1}(N+1)}{\gamma(N-1)} & 0 & \ddots & 0 \\
\vdots & 0 & 0 & 1 \\
\frac{-\alpha_{d_{2}\left(N+d_{2}\right)}}{\gamma(N-1)} & 0 & 0 & 0
\end{array}\right], N \geq 1,
\end{aligned}
$$


and also the analogous sequence of matrices $\underset{N}{\mathbf{b}}(y)$ and $\underline{\mathbf{b}}(y)$ of size $d_{1}+1$

$$
\begin{aligned}
& \underset{N}{\mathbf{b}}(y):= {\left[\begin{array}{cccc}
0 & 1 & 0 & 0 \\
0 & 0 & \ddots & 0 \\
0 & 0 & 0 & 1 \\
\frac{-\beta_{d_{1}}(N)}{\gamma(N)} & \cdots & \frac{-\beta_{1}(N)}{\gamma(N)} \frac{\left(y-\beta_{0}(N)\right)}{\gamma(N)}
\end{array}\right], \quad N \geq d_{1}, } \\
& \underline{\mathbf{b}}(y):=\left[\begin{array}{cccc}
\frac{y-\beta_{0}(N)}{\gamma(N-1)} & 1 & 0 & 0 \\
\frac{-\beta_{1}(N+1)}{\gamma(N-1)} & 0 & \ddots & 0 \\
\vdots & 0 & 0 & 1 \\
\frac{-\beta_{d_{1}\left(N+d_{1}\right)}}{\gamma(N-1)} & 0 & 0 & 0
\end{array}\right], N \geq 1 .
\end{aligned}
$$

The first equations in (2.1.19), 2.1.20) and (2.1.24), (2.1.25) imply the following

Lemma 2.1 The sequences of matrices $\underset{N}{\mathbf{a}}, \underset{N}{\mathbf{b}}$ and $\underline{N}, \underline{\mathbf{a}}, \underline{N}$ implement the shift $N \mapsto N+1$ and $N \mapsto N-1$ in the windows of quasi-polynomials and Fourier-Laplace transforms in the sense that

$$
\begin{array}{ll}
\underset{N}{\mathbf{a}} \Psi(x)=\underset{N+1}{\Psi}(x), & \underline{N}(x)=\underline{N+1}(x) \underline{N} \mathbf{\underline { \mathbf { a } }}, \\
\underset{N}{\mathbf{b}} \underset{N}{\Phi}(y)=\underset{N+1}{\Phi}(y), & \underline{N}(y)=\underline{\Psi}(y) \underline{N} \mathbf{\mathbf { b }} .
\end{array}
$$

and in general

$$
\begin{aligned}
& \underset{N+j}{\Psi}=\underset{N+j-1}{\mathbf{a}} \cdots \underset{N}{\mathbf{a}} \Psi, \quad \underline{\Phi}=\underline{\Phi}_{N}^{N+\underline{\mathbf{a}}^{N-1}} \cdots \underline{\mathbf{a}}, \\
& \underset{N+j}{\Phi}=\underset{N+j-1}{\mathbf{b}} \cdots \underset{N}{\mathbf{b} \Phi}, \quad \underline{\Psi}=\underline{\Psi}^{N+j} \stackrel{\underline{\mathbf{b}}}{\cdots} \cdots \underline{\underline{\mathbf{b}}},
\end{aligned}
$$

where $\stackrel{N}{\Psi}, \stackrel{N}{\Phi}$ here denotes a window in any of the Fourier-Laplace transforms defined in eqs. (2.1.10), (2.1.17).

(In this lemma, and in what follows, if no superscript distinguishing the integration path in the FourierLaplace transform in eqs. (2.1.16), (2.1.17) is present in $\stackrel{N}{\underline{\Psi}}, \stackrel{N}{\underline{\Phi}}$, this means that the result being discussed holds for all cases.)

Equations (2.1.34), (2.1.35) will henceforth be referred to as the "ladder relations". We refer to the process of expressing any $\psi_{n}(x)$ or $\underline{\phi}_{n}(x)$ by means of linear combinations of elements in a specific window with polynomial coefficients, as folding onto the specified window. We also recall that matrices $\underset{N}{\mathbf{a}}(y)$ and $\underline{\mathbf{a}}(y), \underline{\mathbf{b}}(y)$ and $\underline{\mathbf{b}}(y)$ are invertible (see [2]). The opposite shifts are therefore implemented by the inverse matrices and the folding may take place in either direction with respect to polynomial degrees. 
Lemma 2.2 (From 娄], with the adapted notation.) The windows of quasi-polynomials $\underset{N}{\Psi}(x), \underset{N}{\Phi}(y)$ and Fourier-Laplace transforms $\stackrel{N}{\Phi}(x), \stackrel{N}{\Psi}(y)$ satisfy the following differential systems

$$
\begin{aligned}
-\hbar \frac{\partial}{\partial x} \underset{N}{\Psi}(x)=\stackrel{N}{D}_{1}(x) \underset{N}{\Psi}(x), \quad N \geq d_{2}+1, \\
\hbar \frac{\partial}{\partial x} \underline{N}(x)=\stackrel{N}{\Phi}(x) \underline{N}_{1}(x), \quad N \geq d_{2}+1, \\
-\hbar \frac{\partial}{\partial y} \underset{N}{\Phi}(y)=\stackrel{N}{D}_{2}(y) \underset{N}{\Phi}(y), \quad N \geq d_{1}+1, \\
\hbar \frac{\partial}{\partial y} \underline{N}(y)=\stackrel{N}{\Psi}(y) \underline{N}_{2}(y), \quad N \geq d_{1}+1
\end{aligned}
$$

where

$$
\begin{aligned}
& \stackrel{N}{D_{1}}(x): \stackrel{N}{\beta_{-1}} \underset{N-1}{\mathbf{a}}{ }^{-1}+\stackrel{N}{\beta_{0}}+\sum_{j=1}^{d_{1}} \stackrel{N}{\beta_{j}} \underset{N+j-1}{\mathbf{a}} \underset{N+j-2}{\mathbf{a}} \cdots \underset{N}{\mathbf{a}} \in g l_{d_{2}+1}[x] . \\
& \underline{D}_{1}^{N}(x):=\underline{\mathbf{a}}^{-1} \underline{\beta}_{-1}^{N}+\underline{\beta}_{0}^{N}+\sum_{j=1}^{d_{1}} \underline{\underline{\mathbf{a}}}^{N-1} \underline{\underline{\mathbf{a}}}^{N-2} \cdots \underline{\underline{\mathbf{a}}}^{N-j} \underline{\beta}_{j}^{N} \in g l_{d_{2}+1}[x], \\
& \stackrel{N}{\beta_{j}}:=\operatorname{diag}\left[\beta_{j}\left(N+j-d_{2}\right), \beta_{j}\left(N+j-d_{2}+1\right), \ldots, \beta_{j}(N+j)\right], j=0, \ldots d_{1}, \\
& \stackrel{N}{\beta}_{-1}:=\operatorname{diag}\left[\gamma\left(N-d_{2}-1\right), \ldots, \gamma(N-1)\right] \\
& \underline{\beta}_{j}^{N}:=\operatorname{diag}\left(\beta_{j}(N-1), \beta_{j}(N), \ldots \beta_{j}\left(N+d_{2}-1\right)\right), \quad j=0, \ldots, d_{1} . \\
& \underline{\beta}_{-1}^{N}:=\operatorname{diag}\left(\gamma(N-1), \gamma(N), \ldots, \gamma\left(N+d_{2}-1\right)\right) \text {. } \\
& \stackrel{N}{D_{2}}(y):=\stackrel{N}{\alpha}_{-1} \underset{N-1}{\mathbf{b}}{ }^{-1}+\stackrel{N}{\alpha}_{0}+\sum_{j=1}^{d_{2}} \stackrel{N}{\alpha_{j}} \underset{N+j-1}{\mathbf{b}} \underset{N+j-2}{\mathbf{b}} \cdots \underset{N}{\mathbf{b}} \in g l_{d_{1}+1}[y] . \\
& \underline{D}_{2}^{N}(y):=\underline{\mathbf{b}}^{-1} \underline{\underline{\alpha}}_{1}^{N}+\underline{\alpha}_{0}^{N}+\sum_{j=1}^{d_{2}} \underline{\mathbf{b}}^{N-1} \underline{\mathbf{b}} \underline{\mathbf{b}}^{N-2} \cdots \underline{\mathbf{b}}^{N-j} \underline{\underline{\alpha}}_{j}^{N} \in g l_{d_{1}+1}[y], \\
& \stackrel{N}{\alpha_{j}}:=\operatorname{diag}\left[\alpha_{j}\left(N+j-d_{1}\right), \alpha_{j}\left(N+j-d_{1}+1\right), \ldots, \alpha_{j}(N+j)\right], j=0, \ldots d_{2}, \\
& \stackrel{N}{\alpha}_{-1}:=\operatorname{diag}\left[\gamma\left(N-d_{1}-1\right), \ldots, \gamma(N-1)\right] \\
& \stackrel{N}{\alpha_{j}}:=\operatorname{diag}\left(\alpha_{j}(N-1), \alpha_{j}(N), \ldots \alpha_{j}\left(N+d_{1}-1\right)\right), \quad j=0, \ldots, d_{2} . \\
& \underline{N}_{-1}:=\operatorname{diag}\left(\gamma(N-1), \gamma(N), \ldots, \gamma\left(N+d_{1}-1\right)\right) \text {. }
\end{aligned}
$$

Moreover the systems 2.1.38)-(2.1.41) are compatible with the ladder relations (2.1.34), 2.1.35) since

$$
\begin{aligned}
& \stackrel{N+1}{D_{1}}(x)=\underset{N}{\mathbf{a}}(x) \stackrel{N}{D_{1}}(x) \underset{N}{\mathbf{a}^{-1}}(x)-\hbar \underset{N}{\mathbf{a}^{\prime}}(x) \underset{N}{\mathbf{a}^{-1}}(x) \\
& \underline{D}_{1}^{N+1}(x)=\underline{N} \underline{\mathbf{a}}(x) \underline{D}_{1}^{N}(x) \underline{\mathbf{a}}^{-1}(x)-\hbar \underline{\mathbf{a}}^{\prime}(x) \underline{\mathbf{a}}^{N-1}(x) \text {. }
\end{aligned}
$$




$$
\begin{aligned}
& \stackrel{N+1}{D_{2}}(y)=\underset{N}{\mathbf{b}}(y) \stackrel{N}{D_{2}}(y) \underset{N}{\mathbf{b}^{-1}}(y)-\hbar \underset{N}{\mathbf{b}^{\prime}}(y) \underset{N}{\mathbf{b}^{-1}}(y) \\
& \underline{N}_{2}^{N+1}(y)=\underline{N}(y) \underline{D}_{2}(y) \underline{\mathbf{b}}^{-1}(y)-\hbar \underline{\mathbf{b}}^{\prime}(y) \underline{\mathbf{b}}^{-1}(y) .
\end{aligned}
$$

Remark 2.1 We stress that the windows taken from any wave-vector solution to eqs. (2.1.19), (2.1.29) and eqs. 2.1.24), 2.1.25) will automatically satisfy the systems specified in Lemma 2.9 (differential and difference equations).

In the following, we will only consider the fundamental systems of solutions to the sequence of equations (2.1.38), 2.1.39), 2.1.54), 2.1.55), since the corresponding dual sequence of equations for the quantities $\underset{N}{\Phi}(y), \underline{\Psi}(y)$ may be treated analogously by just making the appropriate interchange of notations $x \stackrel{N}{\leftrightarrow} y, \Psi \leftrightarrow \Phi, d_{1} \leftrightarrow d_{2}$, etc., and the interchange of integration contours in the $x$ and $y$ planes.

In [2] it was shown that there exists a natural nondegenerate Christoffel-Darboux pairing between

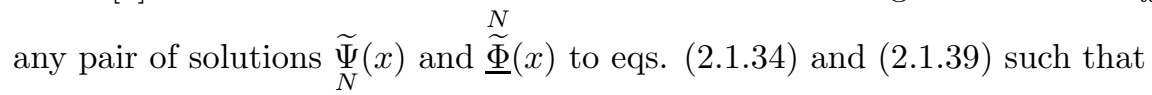

$$
\left(\frac{N}{\widetilde{\Phi}}, \widetilde{\Psi}\right)_{N}:=\underline{\widetilde{\Phi}}(x) \stackrel{N}{\mathbb{A}} \underset{N}{\widetilde{\Psi}}(x),
$$

is constant both in $x$ and $N$, where the invertible matrix $\stackrel{N}{\mathbb{A}}$ defining the pairing is

$$
N=\left[\begin{array}{cccc|c}
0 & 0 & 0 & 0 & -\gamma(N-1) \\
\hline \alpha_{d_{2}}(N) & \cdots & \alpha_{2}(N) & \alpha_{1}(N) & 0 \\
0 & \alpha_{d_{2}}(N+1) & \cdots & \alpha_{2}(N+1) & 0 \\
0 & 0 & \alpha_{d_{2}}(N+2) & \cdots & 0 \\
0 & 0 & 0 & \alpha_{d_{2}}\left(N+d_{2}-1\right) & 0
\end{array}\right] .
$$

This follows from the fact that the matrices $\stackrel{N}{D_{1}}$ and $\underline{N}_{1}$ are conjugate to each other by means of the $\operatorname{matrix} \stackrel{N}{\mathbb{A}}$ (Theorem 4.1 in [2])

$$
\stackrel{N}{\mathbb{A}} \stackrel{N}{D_{1}}(x)=\stackrel{N}{D}_{1}(x) \stackrel{N}{\mathbb{A}}
$$

together with the shift relation (2.1.54). The $\left(d_{2}+1\right) \times\left(d_{2}+1\right)$ matrix $\stackrel{N}{\mathbb{A}}$ is the only nonzero block in the commutator $\left[\prod_{0}^{N-1}, Q\right]$, where $\prod_{0}^{N-1}$ denotes the projector onto the first $N$ basis elements (a "canonical" projector). In the following it is convenient to use the same notation $\stackrel{N}{\mathbb{A}}$ both for the finite matrix and the semi-infinite one.

It was also proved in [2] (Proposition 3.3) that one can choose the windows $\underset{N}{\widetilde{\Psi}}$ and $\underset{\widetilde{\Phi}}{\widetilde{N}}$ as joint solutions of the PDE's following from the infinitesimal changes of the coefficients $\left\{u_{J}\right\}$ and $\left\{v_{K}\right\}$ of the potentials (deformation equations). For this choice, the pairing also becomes independent of these deformation parameters. 


\section{$2.2 \quad$ Fundamental solutions of the $D_{1}$ and $\underline{D}_{1}$ systems}

In this section we explicitly construct solutions of the pairs of dual ODE's defined by the matrices $\stackrel{N}{D_{1}}(x)$ and $\underline{N}_{1}(x)$. In fact these solutions will simultaneously satisfy the deformation equations and the ladder recursion relations in $N$. It will be left to the reader to formulate the corresponding statements for the other pairs $\left(\stackrel{N}{D_{2}}(y), \underline{N}_{2}(y)\right)$, which are essentially the same, mutatis mutandis.

As mentioned in Remark 2.1, taking windows within any wave-vector solution to eqs. (2.1.19), 2.1.24) one obtains solutions to the difference-differential equations in Lemma 2.2. Therefore we could try to construct $d_{2}+1$ such wave-vector solutions in order to obtain a fundamental system for eqs. 2.1.39). On the other hand it will be shown in Proposition 2.1 below that the relations (2.1.24) have precisely $d_{2}$ linearly independent wave-vector solutions (given by the $d_{2}$ Fourier-Laplace transforms (2.1.17)) while relations (2.1.19) have only one solution (given by the quasipolynomials). Therefore we will look for solutions to slightly modified recursion relations differing from (2.1.19), 2.1.25) by changing the initial terms 5 . The solutions to these modified recursion relations $\left\{\tilde{\psi}_{n}(x)\right\}_{n \geq 0}$ or $\left\{\underline{\tilde{\psi}}_{n}(x)\right\}_{n \geq 0}$ will still satisfy the unmodified recursions relations, but only for $n$ large enough. This will provide us with the required solutions of the difference-differential equations of Lemma 2.2.

We start by proving that the Fourier-Laplace transforms and the quasipolynomials are the only wave-vector solutions to eqs. (2.1.19), 2.1.24).

Notational remark. Throughout the remainder of this section, $\underset{\infty}{\Psi}(x)$ and $\underset{\infty}{\Phi}(x)$ will be used generally to denote arbitrary wave-vectors consisting of arbitrary solutions and not just the previously defined quasipolynomials and their Fourier-Laplace transforms.

Proposition 2.1 The semi-infinite systems

$$
\begin{aligned}
& \left\{\begin{array}{l}
x \underset{\infty}{\Psi}(x)=Q \underset{\infty}{\Psi}(x) \\
\hbar \partial_{x} \underset{\infty}{\Psi}(x)=-P \underset{\infty}{\Psi}(x)
\end{array},\right. \\
& \left\{\begin{array}{l}
x \underset{\infty}{\Phi}(x)=\frac{\Phi}{\infty}(x) Q \\
\hbar \partial_{x} \frac{\Phi}{\infty}(x)=\frac{\Phi}{\infty}(x) P
\end{array}\right.
\end{aligned}
$$

have 1 and $d_{2}$ linearly independent solutions, respectively, for the wave-vectors $\Psi_{\infty}(x)$ and $\Phi_{\infty}(x)$. (A similar statement holds for $\underset{\infty}{\Phi}, \frac{\Psi}{\infty}$, as solutions to eqs. 2.1.20) and (2.1.25) with $d_{2} \rightarrow d_{1}$ ).

Proof. The compatibility is guaranteed by the string equation (2.1.23). Recalling [2] that

$$
\left(P-V_{1}^{\prime}(Q)\right)_{\geq 0}=\left(Q-V_{2}^{\prime}(P)\right)_{\leq 0}=0
$$

\footnotetext{
${ }^{5}$ This is not new in the context of prthogonal polynomials, where there exist, besides the orthogonal polynomials, solutions of the second kind. (See e.g. [8, 25]).
} 
we have

$$
\begin{aligned}
& 0=\left[\left(P-V_{1}^{\prime}(Q)\right) \underset{\infty}{\Psi}\right]_{0}=-\hbar \psi_{0}^{\prime}(x)-V_{1}^{\prime}(x) \psi_{0}(x) \\
& 0=\left[\underline{\Phi}\left(Q-V_{2}^{\prime}(P)\right)\right]_{0}=x \underline{\phi}_{0}(x)-V_{2}^{\prime}\left(\hbar \partial_{x}\right) \underline{\phi}_{0}(x) .
\end{aligned}
$$

Thus we have only one solution of the first equation (up to normalization) and $d_{2}$ independent solutions for the second. Using the $x$ recursion relations for the $\psi$ sequence, we can build the rest of the sequence by starting from the first term $\psi_{0}(x)$, since the matrix $Q$ has only one nonzero diagonal above the principal one. On the other hand, using the $\partial_{x}$ recursion relations we can build the rest of the $\underline{\phi}_{n}$ sequence starting from any given solutions $\underline{\phi}_{0}$ of $(2.2 .5)$ because the matrix $P$ has only one diagonal below the main one. Q. E. D.

The solutions of Propostion 2.1 are, up to multiplicative constants, exactly the solutions given by the quasipolynomials for the wave-vector $\underset{\infty}{\Psi}$ and the different possible $\frac{\Phi}{\infty}$ 's corresponding to the different solutions of eq. (2.2.5), which are just the $d_{2}$ different Fourier-Laplace transforms of the quasipolynomials $\phi_{n}(y)$. Indeed, the solutions $\underline{\phi}_{0}(x)$ of eq. (2.2.5) can be expressed by

$$
\underline{\phi}_{0}(x) \propto \int_{\Gamma} \mathrm{d} y \mathrm{e}^{\frac{1}{\hbar}\left(x y-V_{2}(y)\right)},
$$

where $\Gamma$ is any of the contours $\Gamma_{k}^{(y)}$ or a linear combination of them.

As indicated in the introduction to this section, in order to find a fundamental system of solutions to the Difference-Differential equations in Lemma 2.2, we consider systems for the wave-vectors modified by the addition of terms that only change the equations involving the first few entries of the wave-vectors. The recursion relations will therefore remain unchanged for $n$ sufficiently large.

Proposition 2.2 The semi-infinite systems:

$$
\begin{aligned}
& \left\{\begin{array}{l}
x \Psi(x)=Q \underset{\infty}{\Psi}(x)-W_{2}\left(-\hbar \partial_{x}\right) F(x) \\
\hbar \partial_{x} \underset{\infty}{\Psi}(x)=-P \underset{\infty}{\Psi}(x)+F(x)
\end{array},\right. \\
& \left\{\begin{array}{l}
x \underset{\infty}{\Phi}(x)=\frac{\Phi}{\infty}(x) Q+U(x) \\
\hbar \partial_{x} \frac{\Phi}{\infty}(x)=\frac{\Phi}{\infty}(x) P+U(x) W_{1}(x)
\end{array}\right.
\end{aligned}
$$

where

$$
\begin{aligned}
& W_{1}(x):=\frac{V_{1}^{\prime}(Q)-V_{1}^{\prime}(x)}{Q-x}, \quad W_{2}\left(\hbar \partial_{x}\right):=\frac{V_{2}^{\prime}(P)-V_{2}^{\prime}\left(\hbar \partial_{x}\right)}{P-\hbar \partial_{x}}, \\
& F(x):=[f(x), 0,0, \cdots]^{t}, \quad U(x):=[u(x), 0,0, \cdots],
\end{aligned}
$$

both have $d_{2}+1$ linearly independent solutions for the extended unknown wave-vectors $(f(x), \underset{\infty}{\Psi}(x))$ and $\left(u(x), \frac{\Phi}{\infty}(x)\right)$. 
Remark 2.2 The terms in the RHS of eqs. 2.2.7), 2.2.8) which are not present in eqs. (2.2.1), (2.2.7) are semi-infinite vectors with at most $d_{2}$ nonzero entries for (2.2.7) or $d_{1}$ for (2.2.9). Indeed the matrices $W_{1}$ and $W_{2}$ (which are polynomials in $Q$ and $P$ of degrees $d_{1}-1$ and $d_{2}-1$ respectively) have finite band sizes, and hence when acting on the vectors $U(x)$ and $F(x)$, which have only a first nonzero entry, produce a finite number of nonzero entries. As a result, windows taken from any solution of the modified wavevector systems (2.2.7), (2.2.8) will provide solutions of the Difference-Differential systems of Lemma 2.8, but only beyond a minimal $N$ value ( $d_{1}$ or $d_{2}$ respectively).

Proof. The compatibility of these systems is not obvious. We have:

$$
\begin{aligned}
& \hbar \underset{\infty}{\Psi}=\left[\hbar \partial_{x}, x\right] \underset{\infty}{\Psi}=\hbar \partial_{x}\left(\underset{\infty}{\Psi}-W_{2}\left(-\hbar \partial_{x}\right) F\right)-x(-P \underset{\infty}{\Psi}+F)
\end{aligned}
$$

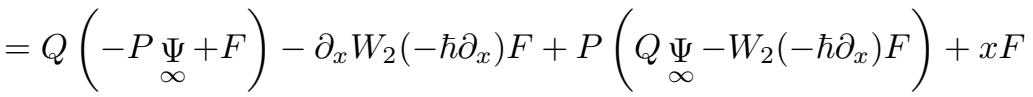

$$
\begin{aligned}
& =[P, Q] \underset{\infty}{\Psi}+Q F-\left(\hbar \partial_{x}+P\right) \frac{V_{2}^{\prime}(P)-V_{2}^{\prime}\left(-\hbar \partial_{x}\right)}{P+\hbar \partial_{x}} F(x)+x F \\
& =\hbar \underset{\infty}{\Psi}+\left(Q-V_{2}^{\prime}(P)\right) F+\left(x-V_{2}^{\prime}\left(-\hbar \partial_{x}\right)\right) F .
\end{aligned}
$$

Since only the first entry of $F$ is nonzero and $Q-V_{2}^{\prime}(P)$ is strictly upper-triangular [2], the second term vanishes. The last term gives the following ODE for the first entry of $F(x)$

$$
V_{2}^{\prime}\left(-\hbar \partial_{x}\right) f(x)=x f(x)
$$

The solutions of eq. (2.2.15) are easily written as Fourier-Laplace integrals, and give a compatible system (see eq. (2.3.16), where we also fix the most convenient normalization for them). We denote them by $f^{(\alpha)}$ with $\alpha=0, \ldots, d_{2}$, with $f^{(0)} \equiv 0$ the trivial solution, corresponding to the unmodified system (2.2.1).

Fixing any such solution $f(x)$, we can now solve for $\Psi$. First of all, one can prove by induction that

$$
Q^{k} \underset{\infty}{\Psi}=x^{k} \underset{\infty}{\Psi}+\frac{Q^{k}-x^{k}}{Q-x} W_{2}\left(-\hbar \partial_{x}\right) F
$$

Next we compute as for the previous proposition

$$
\begin{aligned}
& 0=\left[\left(P-V_{1}^{\prime}(Q)\right) \underset{\infty}{\Psi}\right]_{0}=\left[-\hbar \partial_{x} \underset{\infty}{\Psi}+F-V_{1}^{\prime}(x) \underset{\infty}{\Psi}-\frac{V_{1}^{\prime}(Q)-V_{1}^{\prime}(x)}{Q-x} W_{2}\left(-\hbar \partial_{x}\right) F\right]_{0} \\
& =-\hbar \psi_{0}^{\prime}(x)-V_{1}^{\prime}(x) \psi_{0}(x)+\left[\mathbf{1}-W_{1}(x) W_{2}\left(-\hbar \partial_{x}\right)\right]_{00} f(x)
\end{aligned}
$$

Thus, $\psi_{0}(x)$ must solve this first order system of ODE's. Since there are $d_{2}+1$ choices for the function $f=f^{(\alpha)}$, we correspondingly obtain $d_{2}+1$ independent solutions $\Psi^{(\alpha)}(x)$ to the system. 
Now consider the second system (2.2.8). The compatibility gives

$$
\begin{aligned}
& \hbar \frac{\Phi}{\infty}=\left[\hbar \partial_{x}, x\right] \frac{\Phi}{\infty}=\hbar \partial_{x}\left(\frac{\Phi}{\infty} Q+U\right)-x\left(\frac{\Phi}{\infty} P+U W_{1}(x)\right) \\
& =\left(\frac{\Phi}{\infty} P+U W_{1}(x)\right) Q+\hbar \partial_{x} U-\left(\frac{\Phi}{\infty} Q+U\right) P-U W_{1}(x) x \\
& =\frac{\Phi}{\infty}[P, Q]-U P+U \frac{V_{1}^{\prime}(Q)-V_{1}^{\prime}(x)}{Q-x}(Q-x)+\hbar \partial_{x} U \\
& =\hbar \frac{\Phi}{\infty}-U\left(P-V_{1}^{\prime}(Q)\right)+\left(\hbar \partial_{x}-V_{1}^{\prime}(x)\right) U(x)
\end{aligned}
$$

Since the first entry of $U$ is nonzero and $P-V_{1}^{\prime}(Q)$ is strictly lower-triangular, the second term vanishes. The last term gives the following ODE for the first entry of $U(x)$ :

$$
\hbar u^{\prime}(x)=V_{1}^{\prime}(x) u(x) \Rightarrow u(x)=c \mathrm{e}^{\frac{1}{\hbar} V_{1}(x)} \text { or } u(x) \equiv 0 .
$$

We next consider the solutions $\frac{\Phi}{\infty}$. By a computation similar to the previous one, we have

$$
\begin{aligned}
& 0=\left[\frac{\Phi}{\infty}\left(Q-V_{2}^{\prime}(P)\right)\right]_{0}=\left[x \frac{\Phi}{\infty}-U-V_{2}^{\prime}\left(\hbar \partial_{x}\right) \frac{\Phi}{\infty}+U W_{1}(x) \frac{V_{2}^{\prime}(P)-V_{2}^{\prime}\left(\hbar \overleftarrow{\partial}_{x}\right)}{P-\hbar \overleftarrow{\partial}_{x}}\right]_{0} \\
& =\left(x-V_{2}^{\prime}\left(\hbar \partial_{x}\right)\right) \phi_{0}(x)-u(x)\left[\mathbf{1}-W_{1}(x) W_{2}\left(\hbar \overleftarrow{\partial}_{x}\right)\right]_{00}
\end{aligned}
$$

This is a $d_{2}$-th order inhomogeneous ODE for the function $\underline{\phi}_{0}(x)$. Choosing $u(x) \equiv 0$ as solution to (2.2.25) we have $d_{2}$ independent solutions corresponding to the "unmodified" system 2.2.2). The choice

$$
u(x)=c \mathrm{e}^{\frac{1}{\hbar}} V_{1}(x)
$$

leads to one more independent solution of the inhomogeneous equation. Q. E. D.

We denote the solutions to system $(2.2 .8)$ in general as $\underline{\Phi}^{(\alpha)}, \alpha=0, \ldots d_{2}$, where $\alpha=0$ corresponds to the inhomogeneous solutions and $\alpha=1, \ldots d_{2}$ correspond to the independent solutions to the homogeneous case $(U(x) \equiv 0)$. We will give explicit integral representations for the $d_{2}+1$ solutions in the next section. With the solutions $\underset{\infty}{\Psi^{(\alpha)}}, \underline{\Phi}^{(\beta)}, \alpha, \beta=0, \ldots, d_{2}$, we can construct the $\left(d_{2}+1\right) \times\left(d_{2}+1\right)$ modified kernels

$$
\stackrel{N}{K}_{11}^{(\alpha, \beta)}\left(x, x^{\prime}\right):=\sum_{n=0}^{N-1} \underline{\phi}_{n}^{(\alpha)}(x) \psi_{n}^{(\beta)}\left(x^{\prime}\right)=\underline{\Phi}_{\infty}^{(\alpha)}(x) \prod_{0}^{N-1} \Psi_{\infty}^{(\beta)}\left(x^{\prime}\right) .
$$

and also obtain the following modified Christoffel-Darboux formulae (cf. [2]).

\section{Proposition 2.3 (Christoffel-Darboux Kernels)}

$$
\begin{aligned}
(x & \left.-x^{\prime}\right){ }^{N} K_{11}^{(\alpha, \beta)}\left(x, x^{\prime}\right) \\
& =\left(\frac{\Phi}{\infty}^{(\alpha)}(x) Q+\delta_{\alpha 0} U(x)\right) \prod_{0}^{N-1} \Psi_{\infty}^{(\beta)}\left(x^{\prime}\right)-\frac{\Phi}{\infty}^{(\alpha)}(x) \prod_{0}^{N-1}\left(Q \underset{\infty}{\Psi_{\infty}^{(\beta)}}\left(x^{\prime}\right)-W_{2}\left(-\hbar \partial_{x}^{\prime}\right) F^{(\beta)}\left(x^{\prime}\right)\right) \\
& =-\underline{\Phi}_{\infty}^{(\alpha)}(x)\left[\prod_{0}^{N-1}, Q\right] \underset{\infty}{\Psi^{(\beta)}}\left(x^{\prime}\right)+\delta_{\alpha 0} u(x) \psi_{0}^{(\beta)}\left(x^{\prime}\right)+\underline{\Phi}_{\infty}^{(\alpha)}(x) \underset{0}{\prod_{0}^{N-1}} W_{2}\left(-\hbar \partial_{x}^{\prime}\right) F^{(\beta)}\left(x^{\prime}\right) .
\end{aligned}
$$


Recall that the commutator of the finite band matrix $Q$ with the projector $\prod_{0}^{N-1}$ gives a finite-rank semiinfinite matrix that corresponds to the Christoffel-Darboux kernel matrix $\stackrel{N}{\mathbb{A}}$. As a corollary, we obtain the pairing between the solutions of the systems (2.1.39) by setting $x=x^{\prime}$

\section{Corollary 2.1}

$$
\begin{aligned}
\left(\underline{\Phi}^{(\alpha)},{\underset{\Psi}{N}}^{(\beta)}\right)_{N} & :=\underline{\Phi}^{(\alpha)}(x) \stackrel{N}{\mathbb{A}}{\underset{N}{\Psi}}^{(\beta)}(x)=\underline{\Phi}_{\infty}^{(\alpha)}(x)\left[\prod_{0}^{N-1}, Q\right] \Psi_{\infty}^{(\beta)}(x)= \\
& =\delta_{\alpha 0} u(x) \psi_{0}^{(\beta)}(x)+\underline{\Phi}_{\infty}^{(\alpha)}(x) \prod_{0}^{N-1} W_{2}\left(-\hbar \partial_{x}\right) F^{(\beta)}(x) .
\end{aligned}
$$

As shown in [2], this is a constant (in $x$ ) if $N>d_{2}$. But if $N>d_{2}$ the projector in the second term is irrelevant because the vector $W_{2}\left(-\hbar \partial_{x}\right) F^{(\beta)}(x)$ has only its first $d_{2}+1$ entries nonvanishing, and hence we have, for $N>d_{2}$,

$$
\left(\underline{\Phi}^{(\alpha)},{\underset{N}{\Psi}}^{(\beta)}\right)_{N}=\delta_{\alpha 0} u(x) \psi_{0}^{(\beta)}(x)+\underline{\Phi}_{\infty}^{(\alpha)}(x) \frac{V_{2}^{\prime}(P)-V_{2}^{\prime}\left(-\hbar \partial_{x}\right)}{P+\hbar \partial_{x}} F^{(\beta)}(x) .
$$

If $\alpha \neq 0$ then

$$
\underline{\Phi}_{\infty}^{(\alpha)}(x) P=\hbar \partial_{x} \frac{\Phi}{\infty}^{(\alpha)}(x),
$$

and hence we have, for $N>d_{2}, \quad \alpha \neq 0$,

$$
\begin{aligned}
\underline{\Phi}^{(\alpha)}(x) \underset{\infty}{\mathbb{A}}{\underset{\infty}{\Psi}}^{(\beta)}(x) & =\frac{\Phi^{(\alpha)}(x) \frac{V_{2}^{\prime}\left(\hbar \overleftarrow{\partial}_{x}\right)-V_{2}^{\prime}\left(-\hbar \vec{\partial}_{x}\right)}{\hbar \overleftarrow{\partial}_{x}+\hbar \vec{\partial}_{x}} F^{(\beta)}(x)}{} \\
& =\left.\frac{V_{2}^{\prime}\left(\hbar \partial_{x^{\prime}}\right)-V_{2}^{\prime}\left(-\hbar \partial_{x}\right)}{\hbar \partial_{x^{\prime}}+\hbar \partial_{x}} \underline{\phi}_{0}^{(\alpha)}\left(x^{\prime}\right) f^{(\beta)}(x)\right|_{x^{\prime}=x}
\end{aligned}
$$

In this expression $\underline{\phi}_{0}$ and $f$ are kernel solutions of the pair of adjoint differential equations

$$
\left(V_{2}^{\prime}\left(\hbar \partial_{x}\right)-x\right) \underline{\phi}_{0}^{(\alpha)}(x)=0, \quad\left(V_{2}^{\prime}\left(-\hbar \partial_{x}\right)-x\right) f^{(\beta)}(x)=0,
$$

and the last expression in eq. (2.2.34) is just the bilinear concomitant of the pair (which is a constant in our case). For $\beta=0$ we have

$$
\underline{\Phi}_{\infty}^{(\alpha)}(x) \stackrel{N}{\mathbb{A}} \underset{\infty}{\Psi^{(0)}}(x)=\delta_{\alpha 0} u(x) \psi_{0}^{(0)}(x)=\delta_{\alpha 0} c \mathrm{e}^{\frac{1}{\hbar} V_{1}(x)} \frac{1}{\sqrt{h_{0}}} \mathrm{e}^{-\frac{1}{\hbar} V_{1}(x)}=\delta_{\alpha 0} \frac{c}{\sqrt{h_{0}}} .
$$

For completeness we recall that the matrices $P, Q$ satisfy the following deformation equations

$$
\begin{array}{ll}
\hbar \partial_{u_{K}} Q=-\left[Q, \mathrm{U}^{K}\right], & \hbar \partial_{v_{J}} Q=\left[Q, \mathrm{~V}^{J}\right], \\
\hbar \partial_{u_{K}} P=-\left[P, \mathrm{U}^{K}\right], & \hbar \partial_{v_{J}} P=\left[P, \mathrm{~V}^{J}\right],
\end{array}
$$


where

$$
\mathrm{U}^{K}:=-\frac{1}{K}\left\{\left[Q^{K}\right]_{>0}+\frac{1}{2}\left[Q^{K}\right]_{0}\right\}, \quad \mathrm{V}^{J}:=-\frac{1}{J}\left\{\left[P^{J}\right]_{<0}+\frac{1}{2}\left[P^{J}\right]_{0}\right\} .
$$

(Note that in [2] the matrix that here is denoted by $P$ was denoted $-P$.) Here the subscripts $<0$ (resp. $>0$ ) mean the part of the matrix below (resp., above) the principal diagonal and the subscript 0 denotes the diagonal.

Proposition 2.4 The equations in Proposition 2.9 are compatible with the deformation equations

$$
\begin{aligned}
& \left\{\begin{array}{l}
\hbar \partial_{u_{K}} \underset{\infty}{\Psi}(x)=\mathrm{U}^{K} \underset{\infty}{\Psi}(x) \\
\hbar \partial_{u_{K}} F(x)=\mathrm{U}^{K} F(x)
\end{array}\right. \\
& \left\{\begin{array}{l}
\hbar \partial_{v_{J}} \underset{\infty}{\Psi}(x)=-\mathrm{V}^{J} \underset{\infty}{\Psi}(x)-\frac{1}{J} \frac{P^{J}-\left(-\hbar \partial_{x}\right)^{J}}{P+\hbar \partial_{x}} F(x) \\
\hbar \partial_{v_{J}} F(x)=\left(\frac{\left(-\hbar \partial_{x}\right)^{J}}{J}+\mathrm{V}_{00}^{J}\right) F(x)
\end{array}\right. \\
& \left\{\begin{array}{l}
\hbar \partial_{u_{K}} \frac{\Phi}{\infty}(x)=-\frac{\Phi}{\infty}(x) \mathrm{U}^{K}+\frac{1}{K} U(x) \frac{Q^{K}-x^{K}}{Q-x} \\
\hbar \partial_{u_{K}} U(x)=\left(\frac{x^{K}}{K}+\mathrm{U}_{00}^{K}\right) U(x)
\end{array}\right. \\
& \left\{\begin{array}{l}
\hbar \partial_{v_{J}} \underline{\Phi}(x)=\frac{\Phi}{\infty}(x) \mathrm{V}^{J} \\
\hbar \partial_{v_{J}} U(x)=U(x) \mathrm{V}^{J} .
\end{array}\right.
\end{aligned}
$$

Proof. We only sketch the proof, which is quite straightforward but rather long.

Compatibility of the deformation equations amongst themselves follows from the zero curvature equations

$$
\left[\hbar \partial_{u_{K}}+\mathrm{U}^{K}, \hbar \partial_{u_{K^{\prime}}}+\mathrm{U}^{K^{\prime}}\right]=\left[\hbar \partial_{v_{J}}-\mathrm{V}^{J}, \hbar \partial_{v_{J^{\prime}}}-\mathrm{V}^{J^{\prime}}\right]=\left[\hbar \partial_{u_{K}}+\mathrm{U}^{K}, \hbar \partial_{v_{J}}-\mathrm{V}^{J}\right]=0
$$

(which are established in the general theory [26, 2]), together with the fact that both vectors $U(x)$ and $F(x)$ have, by assumption, nonzero entries only in the first position.

Compatibility with eqs. (2.2.7), (2.2.8) requires some more computation. We first prove the compatibility between eq. (2.2.8) and the two eqs. 2.2.42), (2.2.43). Compatibility between the equations involving multiplication by $x$ and application of $\partial_{v_{J}}$ :

$$
\begin{aligned}
& \hbar x \partial_{v_{J}} \frac{\Phi}{\infty}(x)=\frac{\Phi}{\infty}(x) Q \mathrm{~V}^{J}+U(x) \mathrm{V}^{J} \\
& \hbar \partial_{v_{J}} x \frac{\Phi}{\infty}(x)=\frac{\Phi}{\infty}(x) \mathrm{V}^{J} Q+\frac{\Phi}{\infty}(x)\left[Q, \mathrm{~V}^{J}\right]+U(x) \mathrm{V}^{J}=\text { RHS of }(2.2 .45) .
\end{aligned}
$$

Compatibility between the equation involving multiplication by $x$ and application of $\partial_{u_{K}}$ :

$$
x \hbar \partial_{u_{K}} \frac{\Phi}{\infty}(x)=-\frac{\Phi}{\infty}(x) Q \mathrm{U}^{K}-U(x) \mathrm{U}^{K}+\frac{1}{K} U(x) \frac{Q^{K}-x^{K}}{Q-x} x
$$




$$
\begin{aligned}
& \hbar \partial_{u_{K}} x \frac{\Phi}{\infty}(x)=\hbar \partial_{u_{K}} \frac{\Phi}{\infty}(x) Q-\frac{\Phi}{\infty}(x)\left[Q, \mathrm{U}^{K}\right]+\hbar \partial_{u_{K}} U(x) \\
& =-\frac{\Phi}{\infty}(x) \mathrm{U}^{K} Q+\frac{1}{K} U(x) \frac{Q^{K}-x^{K}}{Q-x} Q-\frac{\Phi}{\infty}(x)\left[Q, \mathrm{U}^{K}\right]+\left(\frac{x^{K}}{K}+\mathrm{U}_{00}^{K}\right) U(x) \\
& =-\frac{\Phi}{\infty}(x) Q \mathrm{U}^{K}+\frac{1}{K} U(x)\left(Q^{K}-x^{K}\right)+\frac{1}{K} U(x) \frac{Q^{K}-x^{K}}{Q-x} x+\left(\frac{x^{K}}{K}+\mathrm{U}_{00}^{K}\right) U(x) \\
& \stackrel{(\star)}{=} \text { RHS of (2.2.47), }
\end{aligned}
$$

where in the step $(\star)$, we have used the fact that (since only the first entry of $U(x)$ is nonzero)

$$
\frac{1}{K} U(x) Q^{K}+\mathrm{U}_{00}^{K} U(x)=-U(x) \mathrm{U}^{K} .
$$

Compatibility between the $\partial_{x}$ and $\partial_{u_{K}}$ equations is a bit more involved:

$$
\begin{aligned}
& \hbar^{2} \partial_{x} \partial_{v_{J}} \frac{\Phi}{\infty}(x)=\hbar \partial_{x} \frac{\Phi}{\infty}(x) \mathrm{V}^{J}=\frac{\Phi}{\infty}(x) P \mathrm{~V}^{J}+U(x) W_{1}(x) \mathrm{V}^{J} \\
& \hbar^{2} \partial_{v_{J}} \partial_{x} \frac{\Phi}{\infty}(x)=\hbar \partial_{v_{J}}\left(\frac{\Phi}{\infty}(x) P+U(x) W_{1}(x)\right)= \\
& \quad=\frac{\Phi}{\infty}(x) \mathrm{V}^{J} P+\frac{\Phi}{\infty}(x)\left[P, \mathrm{~V}^{J}\right]+U(x) \mathrm{V}^{J} W_{1}(x)+U(x)\left[W_{1}(x), \mathrm{V}^{J}\right]=\text { RHS of }(2.2 .53)
\end{aligned}
$$

Finally we have the $\partial_{x}, \partial_{v_{J}}$ compatibility:

$$
\begin{aligned}
& \hbar^{2} \partial_{x} \partial_{v_{J}} \frac{\Phi}{\infty}(x)=\hbar \partial_{x}\left(-\frac{\Phi}{\infty}(x) \mathrm{U}^{K}+\frac{1}{K} U(x) \frac{Q^{K}-x^{K}}{Q-x}\right) \\
& =-\left(\frac{\Phi}{\infty}(x) P+U(x) W_{1}(x)\right) \mathrm{U}^{K}+\frac{1}{K} \hbar \partial_{x} U(x) \frac{Q^{K}-x^{K}}{Q-x}+\frac{1}{K} U(x) \hbar \partial_{x} \frac{Q^{K}-x^{K}}{Q-x} \\
& =-\left(\frac{\Phi}{\infty}(x) P+U(x) W_{1}(x)\right) \mathrm{U}^{K}+\frac{1}{K}\left(\hbar \partial_{x}-V_{1}^{\prime}(x)\right) U(x) \frac{Q^{K}-x^{K}}{Q-x} \\
& +\frac{1}{K} U(x)\left(V_{1}^{\prime}(x)-V_{1}^{\prime}(Q)+V_{1}^{\prime}(Q)-P\right) \frac{Q^{K}-x^{K}}{Q-x} \\
& +\frac{1}{K} U(x) P \frac{Q^{K}-x^{K}}{Q-x}+\frac{1}{K} U(x) \hbar \partial_{x} \frac{Q^{K}-x^{K}}{Q-x} \\
& \stackrel{(\star \star)}{=}-\left(\frac{\Phi}{\infty}(x) P+U(x) W_{1}(x)\right) \mathrm{U}^{K}-\frac{1}{K} U(x) W_{1}(x)\left(Q^{K}-x^{K}\right)+\frac{1}{K} U(x) \frac{Q^{K}-x^{K}}{Q-x} P \\
& +\frac{1}{K}\left(\hbar \partial_{x}-V_{1}^{\prime}(x)\right) U(x) \frac{Q^{K}-x^{K}}{Q-x}+\frac{1}{K} U(x)\left(\hbar \partial_{x}+\hbar \partial_{Q}\right) \frac{Q^{K}-x^{K}}{Q-x} \\
& =-\frac{\Phi}{\infty}(x) P-U(x) W_{1}(x)\left(\frac{1}{K} Q^{K}+\mathrm{U}^{K}\right)+\frac{x^{K}}{K} U(x) W_{1}(x)+\frac{1}{K} U(x) \frac{Q^{K}-x^{K}}{Q-x} P \\
& +\frac{1}{K}\left(\hbar \partial_{x}-V_{1}^{\prime}(x)\right) U(x) \frac{Q^{K}-x^{K}}{Q-x}+\hbar U(x) \frac{Q^{K-1}-x^{K-1}}{Q-x} \\
& =-\frac{\Phi}{\infty}(x) P-U(x)\left(\frac{1}{K} Q^{K}+\mathrm{U}^{K}\right) W_{1}(x)+\frac{x^{K}}{K} U(x) W_{1}(x)+\frac{1}{K} U(x) \frac{Q^{K}-x^{K}}{Q-x} P \\
& +\frac{1}{K}\left(\hbar \partial_{x}-V_{1}^{\prime}(x)\right) U(x) \frac{Q^{K}-x^{K}}{Q-x}+\hbar U(x) \frac{Q^{K-1}-x^{K-1}}{Q-x}-U(x)\left[W_{1}(x), \mathrm{U}^{K}\right]
\end{aligned}
$$




$$
\begin{aligned}
\stackrel{(\star \star \star)}{=}- & \frac{\Phi}{\infty}( \\
& x) P+\left(\frac{x^{K}}{K}+\mathrm{U}_{00}^{K}\right) U(x) W_{1}(x)+\frac{1}{K} U(x) \frac{Q^{K}-x^{K}}{Q-x} P \\
& +\frac{1}{K}\left(\hbar \partial_{x}-V_{1}^{\prime}(x)\right) U(x) \frac{Q^{K}-x^{K}}{Q-x}+\hbar U(x) \frac{Q^{K-1}-x^{K-1}}{Q-x}-U(x)\left[W_{1}(x), \mathrm{U}^{K}\right] .
\end{aligned}
$$

In step $(\star \star)$ we have used the fact that the commutator of $P$ with a function of $Q$ is equivalent to the (formal) derivative $\hbar \partial_{Q}$, while in step $(\star \star \star)$ we have used eq. (2.2.52). Computing the derivatives in the opposite order we obtain

$$
\begin{aligned}
& \hbar^{2} \partial_{v_{J}} \partial_{x} \frac{\Phi}{\infty}(x)=\hbar \partial_{v_{J}}\left(\frac{\Phi}{\infty}(x) P+U(x) W_{1}(x)\right) \\
& =\left(-\frac{\Phi}{\infty}(x) \mathrm{U}^{K}+\frac{1}{K} U(x) \frac{Q^{K}-x^{K}}{Q-x}\right) P+\left(\frac{x^{K}}{K}+\mathrm{U}_{00}^{K}\right) U(x) W_{1}(x) \\
& \quad+\hbar U(x) \frac{x^{K-1}-Q^{K-1}}{x-Q}-U(x)\left[W_{1}(x), \mathrm{U}^{K}\right] .
\end{aligned}
$$

Subtracting eq. (2.2.55) from eq. (2.2.56), we are left with

$$
\begin{aligned}
& 0=\operatorname{RHS} \text { of }(2.2 .55)-\operatorname{RHS} \text { of }(2.2 .56)=\frac{1}{K}\left(\hbar \partial_{x}-V_{1}^{\prime}(x)\right) U(x) \frac{Q^{K}-x^{K}}{Q-x} \\
& \Longrightarrow\left(\hbar \partial_{x}-V_{1}^{\prime}(x)\right) U(x)=0,
\end{aligned}
$$

where the implication follows from the fact that only the first entry of $U(x)$ is nonzero. This is precisely the same compatibility condition that we found in Prop. 2.2. The other compatibility checks are also rather long but routine and are left to the reader to fill in. Q. E. D.

\subsection{Explicit Integral representations for the dual wave-vectors}

Proposition 2.5 The $d_{2}+1$ semi-infinite wave-vectors $\frac{\Phi}{\alpha}^{(\alpha)}$ and functions $u^{(\alpha)}(x), \alpha=0, \ldots d_{2}$ defined by

$$
\begin{aligned}
& \underline{\Phi}^{(0)}(x)=\mathrm{e}^{\frac{1}{\hbar} V_{1}(x)} \int_{\varkappa \Gamma} \mathrm{d} s \mathrm{~d} y \frac{\mathrm{e}^{-\frac{1}{\hbar}\left(V_{1}(s)-s y\right)}}{x-s} \Phi^{t}(y), \quad u^{(0)}(x):=\sqrt{h_{0}} \mathrm{e}^{\frac{1}{\hbar} V_{1}(x)} \\
& \underline{\Phi}^{(k)}(x)=\int_{\Gamma_{k}^{(y)}} \mathrm{d} y \mathrm{e}^{\frac{x y}{\hbar}} \Phi^{t}(y), \quad u^{(k)}(x) \equiv 0, \quad k=1, \ldots, d_{2},
\end{aligned}
$$

are independent solutions of the modified system (2.2.8).

Proof. There is nothing to prove for $\frac{\Phi}{\infty}^{(k)}$, since for $k>0$ these are just the Fourier-Laplace transforms, which satisfy the corresponding unmodified system (2.2.1), which is the same as the modified system with $u(x) \equiv 0$. Let us therefore consider $\frac{\Phi}{\infty}^{(0)}$. We first check the $x$ recurrence relations.

$$
x \frac{\Phi}{\infty}^{(0)}(x)=\mathrm{e}^{\frac{1}{\hbar} V_{1}(x)} \int_{\varkappa \Gamma} \mathrm{d} s \mathrm{~d} y\left[\frac{(x-s) \mathrm{e}^{-\frac{1}{\hbar}\left(V_{1}(s)-s y\right)}}{x-s} \Phi^{t}(y)+\frac{\mathrm{e}^{-\frac{1}{\hbar}\left(V_{1}(s)-s y\right)}}{x-s} s \Phi^{t}(y)\right]
$$




$$
\begin{aligned}
& =\left[\sqrt{h_{0}} \mathrm{e}^{\frac{1}{\hbar} V_{1}(x)}, 0, \ldots\right]-\mathrm{e}^{\frac{1}{\hbar} V_{1}(x)} \int_{\varkappa \Gamma} \mathrm{d} s \mathrm{~d} y \frac{\mathrm{e}^{-\frac{1}{\hbar}\left(V_{1}(s)+s y\right)}}{x-s} \hbar \partial_{y} \Phi^{t}(y) \\
& \stackrel{(\star)}{=} U^{(0)}(x)+\underline{\Phi}^{(0)}(x) Q
\end{aligned}
$$

where in $(\star)$ we have used eq. (2.1.20) and defined the semi-infinite vector

$$
U^{(0)}(x):=\left[\sqrt{h_{0}} \mathrm{e}^{\frac{1}{\hbar} V_{1}(x)}, 0, \ldots\right] .
$$

It is easy to prove by induction in $k$, starting from the first part of eq. (2.2.8), that

$$
x^{k} \frac{\Phi}{\infty}^{(0)}(x)=U^{(0)}(x) \frac{x^{k}-Q^{k}}{x-Q}+\frac{\Phi}{\infty}^{(0)}(x) Q^{k} .
$$

We now consider the $\partial_{x}$ differential recursion. After shifting the derivative in $x$ to one in $s$ inside the integral, and integrating by parts, using eq. (2.3.4), we get

$$
\hbar \partial_{x} \frac{\Phi}{\infty}^{(0)}(x)=U^{(0)}(x) \frac{V_{1}^{\prime}(x)-V_{1}^{\prime}(Q)}{x-Q}+\frac{\Phi}{\infty}^{(0)}(x) P .
$$

This proves that the given integral expression is indeed the additional solution to eq. (2.2.8). Q. E. D.

Remark 2.3 We would also have solutions for any other choice of admissible contours (or linear combinations of them) in eq. (2.3.7). This arbitrariness will be used later.

Remark 2.4 The functions $\underline{\phi}_{n}^{(0)}(x)$ are piecewise analytic functions in each connected component of $\mathbb{C}_{x} \backslash \bigcup_{j=1}^{d_{1}} \Gamma_{j}^{(x)}$, but can be analytically continued from each such connected component to entire functions by deforming the contours of integration.

With regard to the deformation equations studied in Propostion 2.4, we have:

Lemma 2.3 The wave-vectors $\underset{\infty}{\Psi^{(0)}}(x)$ (i.e. the quasipolynomials) and $\underline{\Phi}_{\infty}^{(k)}(x), k=1, \ldots, d_{2}$ (i.e. the Fourier-Laplace transform of the quasipolynomials $\phi_{n}(y)$ ) satisfy the following deformation equations

$$
\begin{aligned}
\hbar \partial_{u_{K}} \Psi_{\infty}^{(0)}(x) & =\mathrm{U}^{K} \Psi_{\infty}^{(0)}(x), \\
\hbar \partial_{v_{J}} \Psi^{(0)}(x) & =-\mathrm{V}^{J} \Psi_{\infty}^{(0)}(x), \\
\hbar \partial_{u_{K}} \underline{\Phi}^{(k)}(x) & =-\underline{\Phi}^{(k)}(x) \mathrm{U}^{K}, \\
\hbar \partial_{v_{J}} \frac{\Phi}{\infty}^{(k)}(x) & =\frac{\Phi}{\infty}^{(k)}(x) \mathrm{V}^{J},
\end{aligned}
$$

Proof. This follows straightforwardly from the two equations (2.2.37), 2.2.38) together with the fact that taking a Fourier-Laplace transform commutes with differentiation with respect to the deformation parameters Q. E. D.

We also remark that the given integral expressions satisfy the modified deformation equations given by the following proposition. 
Proposition 2.6 The dual wave-vectors defined in Proposition 2.5 satisfy the following deformation equations

$$
\begin{aligned}
& \hbar \partial_{u_{K}} \frac{\Phi}{\infty}^{(0)}(x)=-\frac{\Phi}{\infty}^{(0)}(x) \mathrm{U}^{K}+\frac{1}{K} U^{(0)}(x) \frac{x^{K}-Q^{K}}{x-Q} \\
& \hbar \partial_{v_{J}} \frac{\Phi}{\infty}^{(0)}(x)=\frac{\Phi}{\infty}^{(0)}(x) \mathrm{V}^{J},
\end{aligned}
$$

where the semi-infinite vector $U^{(0)}(x)$ is defined in eq. 2.3.3).

Proof. As for the $\left\{v_{J}\right\}$ deformation equations for $\frac{\Phi}{\infty}^{(0)}$, the proof follows from Lemma 2.3 and from the fact that the integral transform defining this wave-vector does not depend on the $v_{J}$ 's. On the other hand, the $u_{K}$ deformations give (again using Lemma 2.3):

$$
\hbar \partial_{u_{K}} \frac{\Phi}{\infty}^{(0)}(x)=-\frac{\Phi}{\infty}^{(0)}(x) \mathrm{U}^{K}+\frac{1}{K} \frac{\Phi}{\infty}^{(0)}(x)\left(x^{K}-Q^{K}\right),
$$

from which the proof follows using eq. (2.3.4). Q. E. D.

Remark 2.5 Note that from the definition of the vector $U(x)$, one can verify directly that it satisfies the deformation equations (2.2.49), 2.2.43) by using the equations for the normalization constant $h_{0}$ :

$$
\begin{aligned}
& h_{0}=\int_{\varkappa \Gamma} \mathrm{d} x \mathrm{~d} y \mathrm{e}^{-\frac{1}{\hbar}\left(V_{1}(x)+V_{2}(y)-x y\right)}, \\
& \frac{\hbar}{2} \partial_{u_{K}} \ln \left(h_{0}\right)=\mathrm{U}_{00}^{K}, \quad \frac{\hbar}{2} \partial_{v_{J}} \ln \left(h_{0}\right)=\mathrm{V}_{00}^{J} .
\end{aligned}
$$

Remark 2.6 In particular, the sequences of functions defined in Proposition 2.5 satisfy identical deformation equations for $N$ large enough $\left(N>d_{1}\right)$ and hence the relevant windows satisfy the full DDD equations specified in 国 for the barred (dual) quantities.

We conclude with a discussion regarding the construction of an integral representation for the wavevectors $\Psi$. For the unmodified system (2.2.1) the solution is explicitly given by the quasipolynomials $\underset{\infty}{\Psi^{(0)}}(x)$. The other solutions of the wave-equations (2.2.7) are in $1-1$ correspondence with the $d_{2}$ solutions of eq. (2.2.15) via eq. (2.2.18). We could try to define an integral representation of the form

$$
\underset{\infty}{\Psi^{(k)}}(x)=\int_{\widetilde{\Gamma}_{k}^{(y)}} \frac{\mathrm{d} y}{2 i \pi \hbar} \mathrm{e}^{\frac{1}{\hbar}\left(V_{2}(y)-y x\right)} \int_{\varkappa \Gamma} \mathrm{d} z \mathrm{~d} t \frac{\mathrm{e}^{-\frac{1}{\hbar}\left(V_{2}(t)-z t\right)}}{t-y} \Psi(z),
$$

corresponding to the following solution of eq. 2.2.15

$$
f^{(k)}(x):=\frac{\sqrt{h_{0}}}{2 i \pi \hbar} \int_{\widetilde{\Gamma}_{k}^{(y)}} \mathrm{d} y \mathrm{e}^{\frac{1}{\hbar}\left(V_{2}(y)-x y\right)} \quad k=1, \ldots, d_{2} .
$$

However formula (2.3.15) is not well-defined, since the inner integral defines only a piecewise analytic function with jump discontinuities along the $\Gamma_{k}^{(y)}$ 's, and each of the anti-wedge contours $\widetilde{\Gamma}_{k}^{(y)}$ crosses 
at least one of the contours $\Gamma_{j}^{(y)}$. One could alternatively take the analytic continuation of the inner double integral from one connected component of $\mathbb{C}_{y} \backslash \bigcup_{k=1}^{d_{2}} \Gamma_{k}^{(y)}$ to an entire function, which amounts to deforming the contours in $\varkappa \Gamma$ to avoid any jump discontinuities. But then the outer integral is in general divergent, due to the behaviour of the integrand as $y \rightarrow \infty$.

Thus, as it stands, eq. 2.3.15 may only be viewed as a formal expression which at best defines a divergent integral. However, treating it naively (as though it were uniformly convergent with respect to the parameters $\left\{u_{J}, v_{K}\right\}$, as well as $x$ ) we would find that it indeed satisfies eqs. (2.2.7) (2.2.40) and (2.2.41). Nonetheless we know that there are solutions $\underset{\infty}{\Psi^{(k)}}$ of eq. 2.2.7) 2.2.40) corresponding to the $f^{(k)}(x)$ defined in (2.3.16), determined recursively from the solutions of eq. (2.2.18). Such solutions are uniquely defined by the choice of a particular solution to eq. 2.2.15), up to the addition of a solution of the homogeneous equation (that is, the quasipolynomials) forming $\underset{\infty}{\Psi^{(0)}}$.

There does exist a way to arrive at convergent integral representations, however, by forming suitable linear combinations of Fourier-Laplace transforms of a piecewise analytic function defined along appropriate contours. This was done for the case of cubic potentials by Kapaev in 21. In Appendix A, it will be indicated how the procedure used there may be extended to polynomial potentials of higher degree. For present purposes, however, we may view the solutions as just defined by the recursion relations (2.2.7), together with eq. (2.2.18). It follows from compatibility that these satisfy the deformation equations as well.

As a further remark, we note that the solutions $\underset{\infty}{\Psi^{(k)}}(\bmod \underset{\infty}{\mathbb{C}} \underset{\infty}{(0)})$ associated to the $f^{(k)}$ s defined by eq. (2.3.16), may be associated to any linear combination of the anti-wedge contours, used as contours of integration in (2.3.16). This freedom will be used in the next section.

\subsection{Diagonalization of the Christoffel-Darboux pairing}

We know from Proposition 2.6 that the windows constructed from the integral representations in Proposition 2.5 provide solutions to the DDD equations for the barred system (for $N>d_{1}$ ). On the other hand, we are guaranteed by Theorem 4.1 and Corollary 4.1 of [2] that the Christoffel-Darboux pairing between such solutions and any solution of the unbarred DDD system does not depend on $x, N$, or the deformation parameters determining the two potentials. It is of interest therefore to compute this pairing for the explicit solutions at hand.

The solutions of the unbarred DDD equations are obtained by taking suitable windows in the wavevector solutions of eq. (2.2.7), consisting of the quasipolynomials $\underset{\infty}{\Psi^{(0)}}(x)$ and the solutions $\underset{\infty}{\Psi^{(k)}}(x)$ associated to the $f^{(k)}$ (eq. 2.3.16). It should be clear that the diagonalization of the pairing depends on a careful choice of the contours of integration in eqs. (2.3.2), 2.3.16). Here we prove that the choice of contours that diagonalizes the pairing (2.1.58) is linked to the notion of dual steepest descent-ascent contours, whose definition is given here and will be needed again in the following section. 
Definition 2.4 The steepest descent contours (SDC's) and the dual steepest ascent contours (SAC's) for integrals of the form

$$
I(x):=\int_{\Gamma} \mathrm{d} y \mathrm{e}^{-\frac{1}{\hbar}\left(V_{2}(y)-x y\right)} H(y), \quad \widetilde{I}(x):=\int_{\widetilde{\Gamma}} \mathrm{d} y \mathrm{e}^{\frac{1}{\hbar}\left(V_{2}(y)-x y\right)} H(y),
$$

respectively, passing through any saddle point $y_{k}(x), k=0 \ldots d_{2}-1$ with $H(y)$ at most of exponential type, are the contours $\gamma_{k}$ and $\tilde{\gamma}_{k}$, respectively, uniquely defined by

$$
\begin{aligned}
& \gamma_{k}:=\left\{y \in \mathbb{C} ; \Im\left(V_{2}(y)-x y\right)=\Im\left(V_{2}\left(y_{k}(x)\right)-x y_{k}(x)\right), \Re\left(V_{2}(y)\right) \underset{\substack{y \rightarrow \infty \\
y \in \gamma_{k}}}{\longrightarrow}+\infty\right\} \\
& \widetilde{\gamma}_{k}:=\left\{y \in \mathbb{C} ; \Im\left(V_{2}(y)-x y\right)=\Im\left(V_{2}\left(y_{k}(x)\right)-x y_{k}(x)\right), \Re\left(V_{2}(y)\right) \underset{\substack{y \rightarrow \infty \\
y \in \widetilde{\gamma}_{k}}}{\longrightarrow}-\infty\right\} .
\end{aligned}
$$

Here $y_{k}(x)$ denotes one of the $d_{2}$ branches of the solution to the algebraic equation

$$
V_{2}^{\prime}(y)=x
$$

which behaves like

$$
y_{k}(x) \underset{x \rightarrow \infty}{\sim}\left(v_{d_{2}+1}\right)^{-\frac{1}{d_{2}}} \omega^{k} x^{\frac{1}{d_{2}}}, \quad \omega:=\mathrm{e}^{\frac{2 i \pi}{d_{2}}},
$$

where $x^{\frac{1}{d_{2}}}$ denotes the principal $d_{2}$-th root of $x$. (Note that the homology class of the SDC's and SAC's becomes a constant for $|x|$ sufficiently large along a generic ray (see Fig. 目). It will be proved in Section 3.7 that the homology class is also locally constant with respect to the angle of the ray.

For the following, we also define the sectors

$$
\begin{aligned}
& \mathcal{S}_{k}:=\left\{x \in \mathbb{C}, \arg (x) \in\left(-\vartheta_{y}+\frac{(2 k-1) \pi}{2\left(d_{2}+1\right)},-\vartheta_{y}+\frac{(2 k+1) \pi}{2\left(d_{2}+1\right)}\right)\right\}, \text { for } d_{2} \text { odd } \\
& \mathcal{S}_{k}:=\left\{x \in \mathbb{C}, \arg (x) \in\left(-\vartheta_{y}+\frac{k \pi}{\left(d_{2}+1\right)},-\vartheta_{y}+\frac{(k+1) \pi}{\left(d_{2}+1\right)}\right)\right\}, \text { for } d_{2} \text { even } \\
& k=0, \ldots, 2 d_{2}+1,
\end{aligned}
$$

(where $\vartheta_{y}$ was defined in eq. (2.1.5)), and denote by $\mathcal{R}_{k}, k=0, \ldots 2 d_{2}+1$ the rays separating them, counting counterclockwise, starting from $\mathcal{S}_{0}$. These will be shown in the following section to be interpretable as Stokes' rays, defining a part of the Stokes' sectors in our Riemann-Hilbert problem. It will be shown in Sect. 3.1 that if $x$ approaches $\infty$ along a ray distinct from the $\mathcal{R}_{k}$ 's, then the homology class of the SDC's and SAC's in Def. 2.4 is well defined. We then have

Proposition 2.7 Fix any (non-Stokes) ray $\{\arg (x)=\alpha=$ const. $\} \neq \mathcal{R}_{k}$ and the corresponding limiting (for large $|x|$ ) homology class of the dual steepest descent-ascent contours $\gamma_{k}$ and $\tilde{\gamma}_{k}, k=1, \ldots d_{2}$ of Def. 2.4. With this choice of the contours of integration, use the SDC's to redefine the Fourier-Laplace 


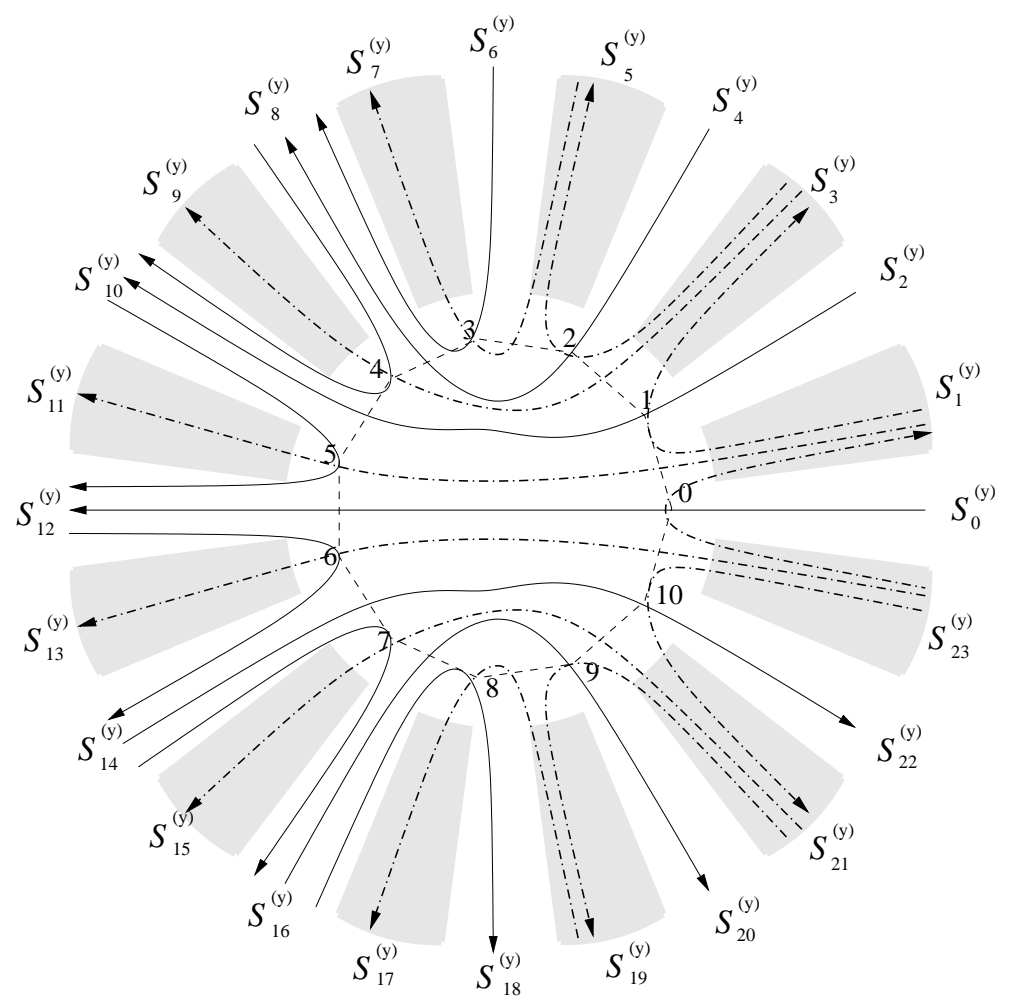

Figure 2: Example of the asymptotic (as $|x| \rightarrow \infty$ ) SDC's $\gamma$ (solid) and SAC's $\tilde{\gamma}$ (line-dot-line) in the $y$-plane for a potential of degree $d_{2}+1=12$ with positive leading coefficient $\left(v_{d_{2}+1}>0\right)$ and for the non-Stokes $\operatorname{line} \arg (x)=0$. The numbers at the vertices of the endecagon label the $d_{2}=11$ critical points and the pair of dual SDC and SAC passing through each them.

transform 2.3.9), and the SAC's to redefine the $f^{(k)} s$ of eq. 2.3.19), and the associated wave-vectors $\Psi^{(k)} \bmod \mathbb{C} \Psi_{\infty}^{(0)}$. Then

$$
C^{\alpha \beta}:=\underline{\Phi}^{(\alpha)}(x) \underset{N}{\mathbb{A}}{\underset{\Psi}{\Psi}}^{(\beta)}(x)=\left[\begin{array}{c|c}
1 & 0 \\
\hline 0 & \mathbf{1}_{d_{2}}
\end{array}\right]=\mathbf{1}_{d_{2}+1}, \quad N>d_{2},
$$

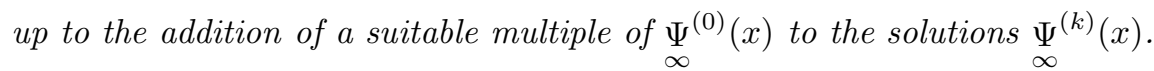

\section{Proof.}

For $\beta=0$ the statement follows from eq. (2.2.36) (where the constant $c$ equals $\sqrt{h_{0}}$ ). For $\alpha=0 \neq \beta$ we use the fact that the solutions $\Psi_{\infty}^{(k)}(x)$ associated to $f^{(k)}$ are defined only up to the addition of a multiple of $\underset{\infty}{\Psi^{(0)}}(x)$. Indeed, we know from the general theory that the pairing of the two solutions is constant, and we may use this freedom to normalize the constant to zero by adding a suitable multiple 
of the homogeneous solution of eq. 2.2.7) $\underset{\infty}{\Psi} \Psi^{(0)}(x)$ (i.e., the quasipolynomials), which is "orthogonal" to the solutions $\underline{\Phi}_{\infty}^{(\alpha)}(x), \alpha \neq 0$, as follows again from eq. (2.2.36).

Finally for $k=\alpha \neq 0 \neq \beta=j, C^{k j}$ equals the bilinear concomitant of the corresponding functions (eq. 2.2.34). Let us consider $x$ belonging to a fixed ray, and choose a basis of steepest descent contours $\gamma_{k}$ and steepest ascent contours $\widetilde{\gamma}_{k}$ (whose homology does not change as $x \rightarrow \infty$ on the fixed ray for $|x|$ big enough). We use formula (2.2.34) with

$$
f^{(j)}(x):=\frac{\sqrt{h_{0}}}{2 i \pi \hbar} \int_{\widetilde{\gamma}_{j}} \mathrm{~d} y \mathrm{e}^{\frac{1}{\hbar}\left(V_{2}(y)-x y\right)}, \quad \underline{\varphi}_{0}^{(k)}(x):=\frac{1}{\sqrt{h_{0}}} \int_{\gamma_{k}} \mathrm{~d} y \mathrm{e}^{-\frac{1}{\hbar}\left(V_{2}(y)-x y\right)} .
$$

The respective asymptotic behaviors for large $|x|$, computed by the saddle-point method, are

$$
\begin{aligned}
& f^{(j)}(x) \simeq \frac{\sqrt{h_{0}}}{2 i \pi \hbar} \mathrm{e}^{\frac{1}{\hbar}\left(V_{2}\left(y_{j}(x)\right)-x y_{j}(x)\right)} \sqrt{\frac{-2 \pi \hbar}{V_{2}^{\prime \prime}\left(y_{j}(x)\right)}\left(1+\mathcal{O}\left(\lambda^{-1}\right)\right)} \\
& \underline{\varphi}_{0}^{(k)}(x) \simeq \frac{1}{\sqrt{h_{0}}} \mathrm{e}^{-\frac{1}{\hbar}\left(V_{2}\left(y_{k}(x)\right)-x y_{k}(x)\right)} \sqrt{\frac{2 \pi \hbar}{V_{2}^{\prime \prime}\left(y_{k}(x)\right)}}\left(1+\mathcal{O}\left(\lambda^{-1}\right)\right),
\end{aligned}
$$

where $y_{k}(x)$ are as in Def. 2.4. The bilinear concomitant is a constant, so it must vanish for $j \neq k$ since the exponential parts of the asymptotic forms in eqs. (2.4.9), 2.4.10) cannot give a nonzero constant when multiplied together.

For $j=k$, the bilinear concomitant is given by the integral

$$
\begin{aligned}
& \left.\frac{V_{2}^{\prime}\left(\hbar \partial_{x^{\prime}}\right)-V_{2}^{\prime}\left(-\hbar \partial_{x}\right)}{\hbar \partial_{x^{\prime}}+\hbar \partial_{x}} \underline{\varphi}_{0}^{(k)}\left(x^{\prime}\right) f^{(k)}(x)\right|_{x^{\prime}=x} \\
& =\frac{1}{2 i \pi \hbar} \int_{\widetilde{\gamma}_{k} \times \gamma_{k}} \mathrm{~d} y \mathrm{~d} y^{\prime} \mathrm{e}^{\frac{1}{\hbar}\left(V_{2}(y)-V_{2}\left(y^{\prime}\right)-x\left(y-y^{\prime}\right)\right)} \frac{V_{2}^{\prime}(y)-V_{2}\left(y^{\prime}\right)}{y-y^{\prime}} \\
& \simeq \frac{1}{2 i \pi \hbar} \mathrm{e}^{\frac{1}{\hbar}\left(V_{2}\left(y_{k}(x)\right)-x y_{k}(x)\right)} \sqrt{\frac{2 \pi \hbar}{V_{2}^{\prime \prime}\left(y_{k}(x)\right)}} \\
& \quad \times \mathrm{e}^{-\frac{1}{\hbar}\left(V_{2}\left(y_{k}(x)\right)-x y_{k}(x)\right)} \sqrt{\frac{-2 \pi \hbar}{V_{2}^{\prime \prime}\left(y_{k}(x)\right)}}\left(1+\mathcal{O}\left(\lambda^{-1}\right)\right) V_{2}^{\prime \prime}\left(y_{k}(x)\right)=1 .
\end{aligned}
$$

This concludes the proof. Q. E. D.

Had we chosen the contours as $\widetilde{\Gamma}_{k}^{(y)}$ and $\Gamma_{k}^{(y)}$ rather than the steepest descent contours, we would have had a constant matrix for $C^{\alpha \beta}$. Notice that the pairing of these integrals is also independent of the deformation parameters determining $V_{1}, V_{2}$, as well as of the choice of the integer $N$ defining the window. As shown in [2], this can always be accomplished through a suitable choice of basis. But here we have explicitly shown how this occurs for the particular normalizations chosen in the integrals.

\section{Asymptotic behavior at infinity and Riemann-Hilbert prob- lem}




\subsection{Stokes sectors and sectorial asymptotics}

Given the duality between the ODE's involving $D_{1}{ }^{N}$ and $\underline{D}_{1}{ }^{N}$ implied by eq. (2.1.60), it is clear that the Stokes matrices around the irregular singularity at $x=\infty$ for systems of the form (2.1.38) and 2.1.39) are related. Using the explicit integral representations of the fundamental solutions, we can determine the asymptotic behavior from these integral representations by saddle point methods. The solutions for the $\underline{N}_{1}$ system are simpler to analyze and do not involve the problems of divergent integrals discussed in the section 2.3 (and the Appendix). But in principle one could consider the asymptotic behavior of the solutions to the system (2.1.38) directly, by taking suitable windows in the wave-vector solutions of eq. (2.2.7).

Remark 3.1 We have seen in Sect. 2.4 that one can choose the wave-vector solutions to eqs. (2.2.7), 2.2.8) so that the Christoffel-Darboux pairing between the dual windows of fundamental solutions implies the identity

$$
\stackrel{N}{\mathbf{\Phi}}(x) \stackrel{N}{\mathbb{A}} \underset{N}{\mathbf{\Psi}}(x)=\mathbf{1}_{d_{2}+1}, \quad N>d_{2} .
$$

Therefore, if we formulate a Riemann-Hilbert problem for $\stackrel{N}{\mathbf{\Phi}}(x)$, we can immediately derive the corresponding one for $\underset{N}{\mathbf{\Psi}}(x)$. Since the asymptotic forms are mutual inverses, the Stokes (and jump) matrices for the one must be the inverses of those for the other. (In our conventions, the Stokes matrices for $\underline{N}(x)$ act on the left, while for $\underset{N}{\mathbf{\Psi}}(x)$ they act on the right.) The formulation for the dual wave-functions is considerably easier, so this is what we analyze here in detail.

From the general theory of ODE's, since the matrix $\underline{N}_{1}^{N}(x)$ is of degree $d_{1}$, one would expect $d_{1}+1$ Stokes sectors. However, this is true only if the leading term of the matrix has a nondegenerate spectrum. In the case at hand, however, we have

$$
\underline{N}_{1}^{N}(x) \sim x^{d_{1}}\left[\begin{array}{l|l}
1 & 0 \\
\hline 0 & 0
\end{array}\right]+\mathcal{O}\left(x^{d_{1}-1}\right) .
$$

Since the spectrum of the leading term has a $d_{2}$-fold degeneracy, we have more complicated asymptotic behavior and the occurrence of more Stokes sectors.

A few more preparatory remarks are required. From the discussion in sections 2.2 and 2.3 we obtain that the fundamental system for the differential-difference equations specified in Lemma 2.2 is provided by

$$
\underline{N}(x):=\left[\begin{array}{c}
N \\
\underline{\Phi}^{(0)}(x) \\
\underline{\Phi}^{(1)}(x) \\
\vdots \\
N \\
\underline{\Phi}^{\left(d_{2}\right)}(x)
\end{array}\right], \quad N \geq d_{1}+1
$$


where $\left\{\underline{\Phi}^{N}(x)\right\}_{k=0, \ldots d_{2}}$ are windows constructed from the wave-vectors defined in Proposition 2.5. Given these integral representations of the solutions, the asymptotic behavior and the Riemann-Hilbert problem can be determined by means of the steepest descent method.

While $\left\{\underline{N}^{(k)}(x)\right\}_{k=1, \ldots d_{2}}$ are entire functions, the integral defining the window $\underline{\Phi}^{(0)}(x)$ defines in fact a piecewise analytic vector function. Its domains of analyticity are the $d_{1}+1$ connected components in which the $x$-plane is partitioned by the contours $\Gamma_{j}^{(x)}, j=1, \ldots d_{1}$. We denote by $\mathcal{D}_{j}$ the connected domain to the right of the contours $\Gamma_{j}^{(x)}$ for $j=1, \ldots, d_{1}$ and by $\mathcal{D}_{0}$ the domain to the left of all contours. (See figure 3 for the case $d_{1}=7$.) The fundamental piecewise-analytic solution $\underline{\boldsymbol{\Phi}}^{N}(x)$ defined in eq.

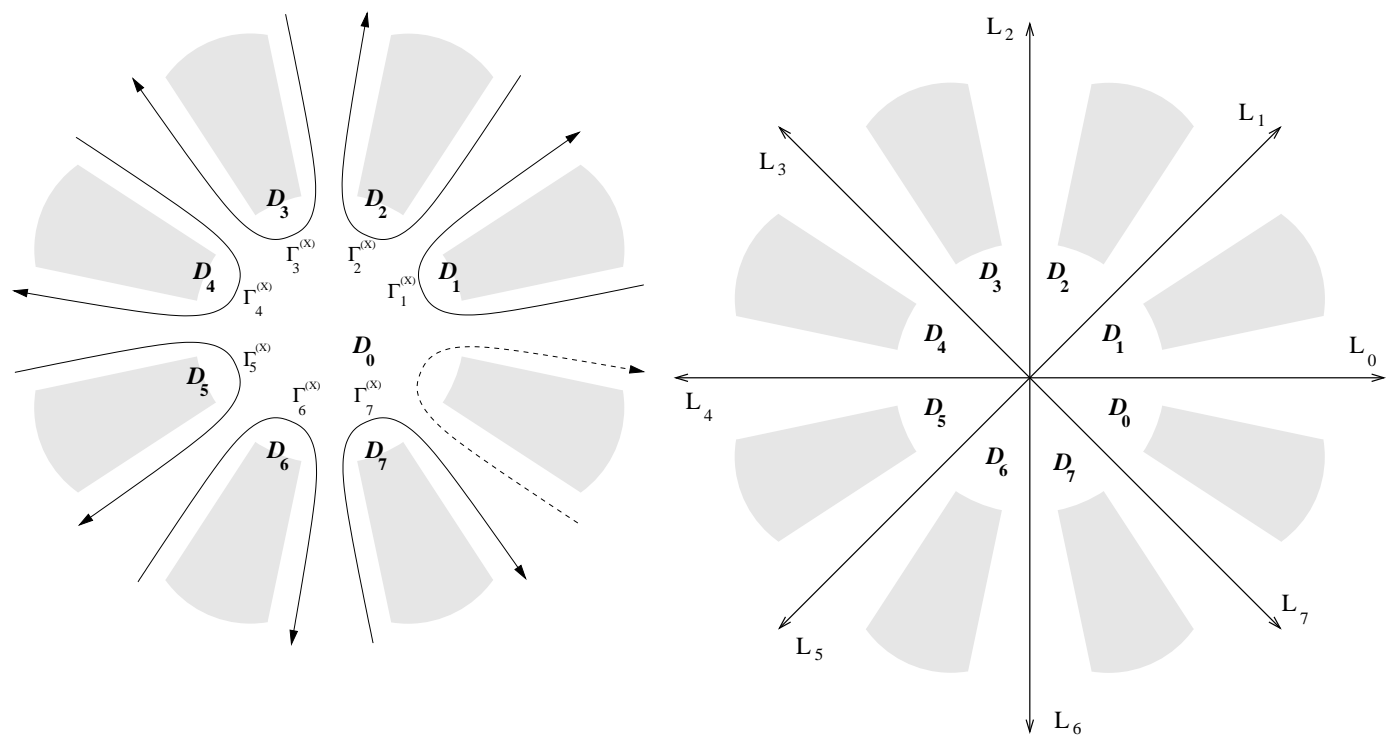

Figure 3: Example of the two possible choices for the domains of definition of $\underline{\phi}_{n}^{(0)}$ (in the $x$-plane) in the case $d_{1}=7$

(3.1.3) then satisfies the jump equations

$$
\underline{\underline{\Phi}}_{+}(x)=\left[\begin{array}{ccccc}
1 & 2 i \pi \varkappa^{\mu, 1} & 2 i \pi \varkappa^{\mu, 2} & \cdots & 2 i \pi \varkappa^{\mu, d_{2}} \\
0 & 1 & 0 & \cdots & 0 \\
0 & 0 & 1 & \cdots & 0 \\
0 & 0 & 0 & \ddots & \\
0 & 0 & 0 & \cdots & 1
\end{array}\right] \underline{\underline{\Phi}}_{-}(x), \quad x \in \Gamma_{\mu}^{(x)}, \mu=1, \ldots, d_{1},
$$

where the subscripts ${ }_{+},-$denote the limiting values from the right or the left, respectively, with respect to the orientation of the contour. Since we can arbitrarily deform the contours in the finite part of the $x$-plane, we can, by retracting the $d_{1}$ contours to the origin arrange that the $d_{1}+1$ regions all become wedge-shaped sectors. Using this freedom in the choice of the contours $\Gamma_{j}^{(x)}$, denote by $L_{\mu} \mu=0, \ldots, d_{1}$ 
the oriented rays starting from the origin and going to infinity in the sectors $\mathcal{S}_{2 \mu}^{(x)}$ defined in 2.1.4). From this point on we choose the contours $\Gamma_{j}^{(x)}$ as follows

$$
\Gamma_{j}^{(x)}:=L_{j}-L_{j-1}, j=1, \ldots, d_{1} .
$$

In this way all the regions, including $\mathcal{D}_{0}$, become wedge-shaped sectors (see Fig. [3). The corresponding (equivalent) jump discontinuities are

$$
\begin{aligned}
& \left.\underline{\boldsymbol{\Phi}}_{+}(x)\right|_{x \in L_{\mu}}=G_{\mu} \underline{\underline{\Phi}}_{-}(x):=\left[\begin{array}{ccccc}
1 & 2 i \pi J_{\mu, 1} & 2 i \pi J_{\mu, 2} & \cdots & 2 i \pi J_{\mu, d_{2}} \\
0 & 1 & 0 & \cdots & 0 \\
0 & 0 & 1 & \cdots & 0 \\
0 & 0 & 0 & \ddots & \\
0 & 0 & 0 & \cdots & 1
\end{array}\right] \underline{\boldsymbol{\Phi}}_{-}(x) \\
& J_{\mu, j}:=\varkappa^{\mu, j}-\varkappa^{\mu+1, j}, \mu=0, \ldots, d_{1}, j=1, \ldots, d_{2}, \varkappa^{0, j}:=\varkappa^{d_{1}+1, j}:=0
\end{aligned}
$$

In order to formulate the complete $\mathrm{RH}$ problem, we need to supplement this discontinuity data with the sectorial asymptotics around the irregular singularity at $x=\infty$ and the Stokes matrices. In doing so one should be careful that the lines $L_{\mu}$ for which the discontinuities are defined do not coincide with any of the Stokes' lines. We can always arrange this by perturbing the rays $L_{\mu}$ within the same sector $\mathcal{S}_{2 \mu}^{(x)}$.

It should be clear that each of the piecewise analytic functions $\underline{N} \underline{N}(x)$ can alternatively be analytically continued to entire functions, since the contours $\Gamma_{j}^{(x)}$ can be deformed arbitrarily in the finite part of the $x$-plane. Therefore the "discontinuities" in the definition of the Hilbert integral are just apparent and have an intrinsic meaning only when studying the asymptotic behavior at infinity. Indeed in the final formulation of our Riemann-Hilbert problem we prefer to let the lines $L_{\mu}$ also play the roles of Stokes' lines.

Proposition 3.1 (Sectorial Asymptotics) In each of the sectors around $x=\infty$ with boundaries given by the Stokes' lines $L_{\mu}, \mu=0, \ldots, d_{1}$ and $\mathcal{R}_{k}, k=0, \ldots 2 d_{2}+1$ (defined right after (2.4.0) the system

$$
\hbar \frac{d}{d x} \stackrel{N}{\underline{\Phi}}(x)=\underline{N}(x) \underline{D}_{1}^{N}(x)
$$

possesses a solution whose leading asymptotic form at $x=\infty$ coincides within this sector with the following formal asymptotic expansion:

$$
\underline{\Phi}_{f o r m}^{N}(x) \sim \mathrm{e}^{\frac{1}{\hbar} T(x)} W x^{G} Y\left(x^{\frac{1}{\sigma_{2}}}\right)
$$

where $Y=Y_{0}+\mathcal{O}\left(x^{-1 / d_{2}}\right)$ is a matrix-valued function analytic at infinity, $Y_{0}$ is a diagonal invertible matrix (specified in the proof) and

$$
T(x):=\sum_{j=0}^{d_{2}} \frac{d_{2} t_{j}}{d_{2}-j+1} x^{\frac{d_{2}+1-j}{d_{2}}} \Omega^{d_{2}+1-j}+V_{1}(x) E
$$




$$
\begin{aligned}
& W:=\left[\begin{array}{c|cccc}
1 & 0 & \ldots & 0 & 0 \\
\hline 0 & \omega & \omega^{2} & \cdots & \omega^{d_{2}} \\
0 & \omega^{2} & \omega^{4} & \cdots & \omega^{2 d_{2}} \\
\vdots & & & \vdots & \\
0 & \omega^{d_{2}} & \omega^{2 d_{2}} & \cdots & \omega^{d^{2}}{ }^{2}
\end{array}\right] \\
& G:=\operatorname{diag}\left(-N, \frac{N+\frac{1}{2}-\frac{d_{2}}{2}}{d_{2}}, \frac{N+\frac{3}{2}-\frac{d_{2}}{2}}{d_{2}}, \ldots, \frac{N-\frac{1}{2}+\frac{d_{2}}{2}}{d_{2}}\right) \\
& \Omega:=\operatorname{diag}\left(0, \omega, \omega^{2}, \ldots, \omega^{d_{2}-1}, \omega^{d_{2}}\right), \omega:=\mathrm{e}^{\frac{2 i \pi}{d_{2}}} \\
& E:=\operatorname{diag}(1,0, \ldots, 0) \\
& t_{0}:=\left(v_{d_{2}+1}\right)^{-\frac{1}{d_{2}}}, \quad t_{1}:=-\frac{1}{d_{2}} \frac{v_{d_{2}}}{v_{d_{2}+1}}, \\
& t_{j}:=\frac{1}{j-1} \underset{y=\infty}{\operatorname{res}}\left(V_{2}^{\prime}(y)\right)^{\frac{j-1}{d_{2}}} \mathrm{~d} y \quad j=2, \ldots, d_{2} .
\end{aligned}
$$

Proof. In any given sector $\mathcal{S}_{k}$ bounded by the lines $\mathcal{R}_{k-1}$ and $\mathcal{R}_{k}$ (eq. 2.4.6) we can choose a basis of steepest descent contours $\gamma_{k}, k=1, \ldots, d_{2}$. The reason why the $\mathcal{S}_{k}$ 's are Stokes sectors and the proper construction of the steepest descent contours is delayed to the discussion of the Stokes' matrices in Sect. 3.2. We Fourier-Laplace transform the quasipolynomials $\phi_{n}(y)$ along these contours in order to obtain the functions $\underline{\phi}_{n}(x)$. Notice that they are not necessarily the same as the previously introduced $\underline{\phi}_{n}^{(k)}(x)$ 's since the steepest descent contours do not necessarily coincide with the contours $\Gamma_{k}^{(y)}$ defined previously. However they are suitable linear combinations with integer coefficients of such $\underline{\phi}_{n}^{(k)}(x)$ 's since the choice of the steepest descent contours is just a different basis in the homology space of the $y$-plane. Now consider the asymptotic expansions for

$$
\underline{\varphi}_{n}^{(k)}(x):=\int_{\gamma_{k}} \mathrm{~d} y \mathrm{e}^{-\hbar^{-1}\left(V_{2}(y)-x y\right)} \phi_{n}(y) .
$$

Here we use the notation $\varphi$ rather than $\phi$ to stress that these are Fourier-Laplace transforms along contours of a homology class equivalent to SDC's. The leading asymptotic term in the sector $\mathcal{S}_{k}$ is given at the critical point of the exponent $V_{2}(y)-x y$ corresponding to the steepest descent contour $\gamma_{k}$. That is, we must compute $V_{2}(y)-x y$ near a solution to:

$$
V_{2}^{\prime}(y)-x=0
$$

asymptotically as $x \rightarrow \infty$ within the specified sector. Let us solve eq.3.1.17) in a series expansion in the local parameter at $\infty$ given by one determination, $\lambda$, of the $d_{2}$-th root of $x$ :

$$
\begin{aligned}
& v_{d_{2}+1} y^{d_{2}}+v_{d_{2}} y^{d_{2}-1}+\ldots=V_{2}^{\prime}(y)=x:=\lambda^{d_{2}} \\
& y(\lambda)=\lambda \sum_{j=0}^{\infty} t_{j} \lambda^{-j} .
\end{aligned}
$$


We then have the formulae (recalling $\lambda=\left(V_{2}^{\prime}(y)\right)^{\frac{1}{d_{2}}}$ )

$$
\begin{aligned}
& t_{0}=\left(v_{d_{2}+1}\right)^{-\frac{1}{d_{2}}}, \\
& t_{1}=\underset{\lambda=\infty}{\operatorname{res}} y(\lambda) \frac{\mathrm{d} \lambda}{\lambda}=\frac{1}{d_{2}} \underset{y=\infty}{\operatorname{res}} y \frac{V_{2}^{\prime \prime}(y)}{V_{2}^{\prime}(y)} \mathrm{d} y=-\frac{1}{d_{2}} \frac{v_{d_{2}}}{v_{d_{2}+1}} \\
& t_{j}=\underset{\lambda=\infty}{\operatorname{res}} \frac{\lambda^{j-1}}{j-1} y^{\prime}(\lambda) \mathrm{d} \lambda=\frac{1}{j-1} \underset{y=\infty}{\operatorname{res}}\left(V_{2}^{\prime}(y)\right)^{\frac{j-1}{d_{2}}} \mathrm{~d} y, j=2 \ldots \infty .
\end{aligned}
$$

As before, denote by $\left\{y_{k}(x)\right\}_{k=0, \ldots d_{2}+1}$ the $d_{2}$ solutions of the equation $V_{2}^{\prime}(y)=x$, which are solved in (3.1.20) by a series in the $d_{2}$-th root of $x$. We then have in a neighborhood of $x=\infty$

$$
V_{2}\left(y_{k}(x)\right)-x y_{k}(x)=-\int^{x} y_{k}\left(x^{\prime}\right) \mathrm{d} x^{\prime}=-d_{2} \int^{\lambda_{k}} y\left(\lambda^{\prime}\right) \lambda^{\prime d_{2}-1} \mathrm{~d} \lambda^{\prime}=-\sum_{j=0}^{\infty} \frac{d_{2} t_{j}}{d_{2}+1-j} \lambda_{k}^{d_{2}+1-j}-c_{k},
$$

where $\lambda_{k}:=\omega^{k} \lambda$ and $c_{k}$ is a constant depending only on the coefficients of $V_{2}$ and the branch of the solution $y_{k}(x)$. This formula is proved by taking the derivative (with respect to $x$ ) of both sides and using the defining equation for $y_{k}(x)$. Notice that there is no logarithmic contribution since $t_{d_{2}+1}=0$ as follows immediately from the residue formula (3.1.20). The different saddle points are computed by replacing $\lambda$ with $\lambda_{k}:=\omega^{k} \lambda$. Substituting into the integral representation of the functions $\underline{\varphi}_{n}^{(k)}(x)$, we get

$$
\begin{aligned}
& \underline{\varphi}_{n}^{(k)}(x):=\frac{1}{\sqrt{h_{n}}} \int_{\gamma_{k}} \mathrm{~d} y \mathrm{e}^{-\frac{1}{\hbar}\left(V_{2}(y)-x y\right)} \sigma_{n}(y) \\
& \simeq \frac{\mathrm{e}^{\frac{c_{k}}{\hbar}}}{\sqrt{h_{n}}} \mathrm{e}^{\frac{1}{\hbar} \int^{x} y_{k}\left(x^{\prime}\right) \mathrm{d} x^{\prime}} \sigma_{n}\left(y\left(\lambda_{k}\right)\right) \int_{\mathbb{R}} \mathrm{e}^{-\frac{1}{2 \hbar} V_{2}^{\prime \prime}\left(y\left(\lambda_{k}\right)\right) t^{2}} \mathrm{~d} t \\
& =\frac{\mathrm{e}^{\frac{c_{k}}{\hbar}}}{\sqrt{h_{n}}} \mathrm{e}^{\mathrm{e}^{x} y_{k}\left(x^{\prime}\right) \mathrm{d} x^{\prime}} \sigma_{n}\left(y\left(\lambda_{k}\right)\right) \sqrt{\frac{2 \pi \hbar}{V_{2}^{\prime \prime}\left(y\left(\lambda_{k}\right)\right)}}
\end{aligned}
$$

Differentiating $V_{2}^{\prime}\left(y\left(\lambda_{k}\right)\right)=\lambda_{k}{ }^{d_{2}}$ implicitly we obtain the relation

$$
V_{2}^{\prime \prime}\left(y\left(\lambda_{k}\right)\right)=\frac{d_{2} \lambda_{k}{ }^{d_{2}-1}}{y^{\prime}\left(\lambda_{k}\right)}
$$

where $y^{\prime}(\lambda)$ means differentiation with respect to $\lambda$. Therefore we obtain

$$
\begin{aligned}
& \simeq \overbrace{}^{\sqrt{\frac{2 \pi \hbar}{h_{n}}} \lambda_{k}{ }^{n-\frac{d_{2}-1}{2}} v_{d_{2}+1}^{-\frac{2 n-1}{2 d_{2}}}} \\
& \underline{\varphi}_{n}^{(k)}(x) \sim \mathrm{e}^{-\hbar^{-1}\left(V_{2}\left(y_{k}(x)\right)-x y_{k}(x)\right)} \overbrace{\frac{\sigma_{n}\left(y_{k}(x)\right)}{\sqrt{h_{n}}} \sqrt{\frac{2 \pi \hbar}{V_{2}^{\prime \prime}\left(y_{k}(x)\right)}}}^{h^{h_{n}}} \\
& \sim \sqrt{\frac{2 \pi \hbar}{d_{2} h_{n}}} \mathrm{e}^{-\hbar^{-1}\left(V_{2}\left(y_{k}(x)\right)-x y_{k}(x)\right)} \lambda_{k}{ }^{n-\frac{d_{2}-1}{2}} v_{d_{2}+1}^{-\frac{2 n-1}{2 d_{2}}} \\
& =\frac{1}{\sqrt{h_{n}}} \mathrm{e}^{\frac{1}{\hbar} \int^{x} y_{k}\left(x^{\prime}\right) \mathrm{d} x^{\prime}} \sigma_{n}\left(y\left(\lambda_{k}\right)\right) \sqrt{\frac{2 \pi \hbar y^{\prime}\left(\lambda_{k}\right)}{d_{2} \lambda_{k} d_{2}-1}} \\
& =C_{k} \frac{v_{d_{2}+1}{ }^{-\frac{n+1}{d_{2}}}}{\sqrt{h_{n}}} \lambda_{k}{ }^{n+\frac{1-d_{2}}{2}} \exp \left[\frac{1}{\hbar} \sum_{j=0}^{d_{2}} \frac{d_{2} t_{j}}{d_{2}+1-j} \lambda_{k}{ }^{d_{2}+1-j}\right]\left(1+\mathcal{O}\left(\lambda^{-1}\right)\right) \text {, }
\end{aligned}
$$


where

$$
C_{k}:=\mathrm{e}^{\frac{c_{k}}{\hbar}} \sqrt{\frac{2 \pi \hbar}{v_{d_{2}+1}^{\frac{1}{d_{2}}} d_{2}}} .
$$

Note that the full series (3.1.21) which should appear in the exponent of $(3.1 .28)$ has been truncated to $j \leq d_{2}$ because the terms corresponding to $j>d_{2}+1$ contribute to negative powers in the exponential and hence give a $\left(1+\mathcal{O}\left(\lambda^{-1}\right)\right)$ term and, as remarked above, there is no term for $j=d_{2}+1$ since $t_{d_{2}+1}=0$.

On the other hand the functions $\phi_{n}^{(0)}(x)$ have the following asymptotic expansion as $x \rightarrow \infty$ within the $\mathcal{D}_{\mu}$ sectors:

$$
\begin{aligned}
& \phi_{n}^{(0)}(x):=\mathrm{e}^{\frac{1}{\hbar} V_{1}(x)} \int_{\varkappa \Gamma} \mathrm{d} s \mathrm{~d} y \frac{\mathrm{e}^{-\hbar^{-1}\left(V_{1}(s)-s y\right)} \phi_{n}(y)}{(x-s)} \\
& \simeq \mathrm{e}^{\frac{1}{\hbar} V_{1}(x)} \sum_{k=0}^{\infty} x^{-k-1} \int_{\varkappa \Gamma} \mathrm{d} s \mathrm{~d} y s^{k} \mathrm{e}^{-\hbar^{-1} V_{1}(s)} \underline{\phi}_{n}(y) \\
& =\sqrt{h_{n}} x^{-n-1} \mathrm{e}^{\hbar^{-1} V_{1}(x)}\left(1+\mathcal{O}\left(x^{-1}\right)\right) .
\end{aligned}
$$

Therefore the matrix of leading terms of $\stackrel{N}{\Phi}(x)$ as defined in eq. (3.1.3) is given by

$$
\begin{aligned}
& \operatorname{diag}\left(1, C_{1}, \cdots C_{d_{2}}\right) \mathrm{e}^{\frac{1}{\hbar} T(x)}\left[\begin{array}{ccc}
h_{N-1} v_{d_{2}+1}^{\frac{N}{d_{2}}} x^{-N} & \cdots & h_{N+d_{2}-1} v_{d_{2}+1}^{\frac{N+d_{2}}{d_{2}}} x^{-N-d_{2}} \\
\lambda_{1}{ }^{N-\frac{1}{2}-\frac{d_{2}}{2}} & \cdots & \lambda_{1}{ }^{N-\frac{1}{2}+\frac{d_{2}}{2}} \\
\lambda_{2}{ }^{N-\frac{1}{2}-\frac{d_{2}}{2}} & \cdots & \lambda_{2}{ }^{N-\frac{1}{2}+\frac{d_{2}}{2}} \\
\vdots & \cdots & \vdots \\
\lambda_{d_{2}}{ }^{N-\frac{1}{2}-\frac{d_{2}}{2}} & \cdots & \lambda_{d_{2}}{ }^{N-\frac{1}{2}+\frac{d_{2}}{2}}
\end{array}\right] . \\
& \cdot \operatorname{diag}\left(\frac{v_{d_{2}+1}^{-\frac{N}{d_{2}}}}{\sqrt{h_{N-1}}}, \ldots, \frac{v_{d_{2}+1}^{-\frac{N+d_{2}}{d_{2}}}}{\sqrt{h_{N+d_{2}-1}}}\right) \text {. }
\end{aligned}
$$

The determinant of the Vandermonde-like matrix is very simple: by computing it along the first row one realizes that only the first and last minors are not zero. Indeed for all other minors the corresponding submatrix has the first and last column proportional. The first minor is a constant in $x$ while the last is of order $x^{-d_{2}}$. Therefore we can write

$$
\left[\begin{array}{ccc}
h_{N-1} v_{d_{2}+1}{ }^{\frac{N}{d_{2}}} x^{-N} & \cdots & h_{N+d_{2}-1} v_{d_{2}+1} \frac{N+d_{2}}{d_{2}} x^{-N-d_{2}} \\
\lambda_{1}{ }^{N-\frac{1}{2}-\frac{d_{2}}{2}} & \cdots & \lambda_{1}{ }^{N-\frac{1}{2}+\frac{d_{2}}{2}} \\
\lambda_{2}{ }^{N-\frac{1}{2}-\frac{d_{2}}{2}} & \cdots & \lambda_{2}{ }^{N-\frac{1}{2}+\frac{d_{2}}{2}} \\
\vdots & \cdots & \vdots \\
\lambda_{d_{2}}{ }^{N-\frac{1}{2}-\frac{d_{2}}{2}} & \cdots & \lambda_{d_{2}}{ }^{N-\frac{1}{2}+\frac{d_{2}}{2}}
\end{array}\right]
$$




$$
\begin{aligned}
& =\left[\begin{array}{cccc}
h_{N-1} v_{d_{2}+1}{ }^{\frac{N}{d_{2}}} x^{-N} & 0 & \cdots & 0 \\
0 & \lambda_{1}{ }^{N+\frac{1}{2}-\frac{d_{2}}{2}} \cdots & \lambda_{1}{ }^{N-\frac{1}{2}+\frac{d_{2}}{2}} \\
0 & \lambda_{2}{ }^{N-\frac{1}{2}-\frac{d_{2}}{2}} \cdots & \lambda_{2}{ }^{N-\frac{1}{2}+\frac{d_{2}}{2}} \\
\vdots & & \cdots & \vdots \\
0 & \lambda_{d_{2}}{ }^{N-\frac{1}{2}-\frac{d_{2}}{2}} \cdots & \lambda_{d_{2}}{ }^{N-\frac{1}{2}+\frac{d_{2}}{2}}
\end{array}\right]\left(1+\mathcal{O}\left(x^{-1}\right)\right) \\
& =\left[\begin{array}{cccc}
h_{N-1} v_{d_{2}+1}{ }^{\frac{N}{d_{2}}} x^{-N} & 0 & \cdots & 0 \\
0 & \lambda_{1}{ }^{N+\frac{1}{2}-\frac{d_{2}}{2}} & \cdots & \lambda_{1}{ }^{N-\frac{1}{2}+\frac{d_{2}}{2}} \\
0 & \lambda_{2}{ }^{N-\frac{1}{2}-\frac{d_{2}}{2}} & \cdots & \lambda_{2}{ }^{N-\frac{1}{2}+\frac{d_{2}}{2}} \\
\vdots & & \cdots & \vdots \\
0 & \lambda_{d_{2}}{ }^{N-\frac{1}{2}-\frac{d_{2}}{2}} & \cdots & \lambda_{d_{2}}{ }^{N-\frac{1}{2}+\frac{d_{2}}{2}}
\end{array}\right]\left(1+\mathcal{O}\left(x^{-1}\right)\right) \\
& =\left[\begin{array}{cccc}
1 & 0 & \cdots & 0 \\
0 & \omega^{N+\frac{1}{2}-\frac{d_{2}}{2}} & \cdots & \omega^{N-\frac{1}{2}+\frac{d_{2}}{2}} \\
0 & \left(\omega^{2}\right)^{N+\frac{1}{2}-\frac{d_{2}}{2}} & \cdots & \left(\omega^{2}\right)^{N-\frac{1}{2}+\frac{d_{2}}{2}} \\
\vdots & & \cdots & \vdots \\
0 & 1 & \cdots & 1
\end{array}\right] \\
& \times \operatorname{diag}\left(h_{N-1} v_{d_{2}+1} \frac{N}{d_{2}} x^{-N}, \lambda^{N+\frac{1}{2}-\frac{d_{2}}{2}}, \ldots, \lambda^{N-\frac{1}{2}+\frac{d_{2}}{2}}\right)\left(1+\mathcal{O}\left(x^{-1}\right)\right)
\end{aligned}
$$

When inserting this into the asymptotic form, we see that, up to factoring the constant invertible (diagonal) matrix on the left

$$
\operatorname{diag}\left(1, C_{1} \omega^{N+\frac{1}{2}-\frac{d_{2}}{2}}, C_{2} \omega^{2\left(N+1 / 2-d_{2} / 2\right)}, \cdots, C_{d_{2}}\right)
$$

which is irrelevant for the asymptotics and depends on $N$ in a rather trivial manner, we obtain a solution with the asymptotic form

$$
\begin{aligned}
& \underline{\underline{\Phi}}_{f o r m}(x) \simeq \exp \frac{1}{\hbar}\left(E V_{1}(x)+\sum_{j=0}^{d_{2}} \frac{d_{2} t_{j} \lambda^{d_{2}+1-j}}{d_{2}-j+1} \Omega^{d_{2}+1-j}\right) W \\
& \times \operatorname{diag}\left(h_{N-1} v_{d_{2}+1} \frac{N}{d_{2}} x^{-N}, \lambda^{N+\frac{1}{2}-\frac{d_{2}}{2}}, \ldots, \lambda^{N-\frac{1}{2}+\frac{d_{2}}{2}}\right) \operatorname{diag}\left(\frac{v_{d_{2}+1}-\frac{N}{d_{2}}}{\sqrt{h_{N-1}}}, \ldots, \frac{v_{d_{2}+1}-\frac{N+d_{2}}{d_{2}}}{\sqrt{h_{N+d_{2}-1}}}\right)\left(1+\mathcal{O}\left(\lambda^{-1}\right)\right) \\
& =\mathrm{e}^{\frac{1}{\hbar} T(x)} W \operatorname{diag}\left(\sqrt{h_{N-1}} x^{-N}, \frac{v_{d_{2}+1}-\frac{N+1}{d_{2}} \lambda^{N+\frac{1}{2}-\frac{d_{2}}{2}}}{\sqrt{h_{N}}}, \ldots, \frac{v_{d_{2}+1}-\frac{N+d_{2}}{d_{2}} \lambda^{N-\frac{1}{2}+\frac{d_{2}}{2}}}{\sqrt{h_{N+d_{2}-1}}}\right)\left(1+\mathcal{O}\left(\lambda^{-1}\right)\right) \\
& =\exp \left(\frac{1}{\hbar} W T(x) W^{-1}\right) x^{G} \operatorname{diag}\left(\sqrt{h_{N-1}}, \frac{v_{d_{2}+1}-\frac{N+1}{d_{2}}}{\sqrt{h_{N}}}, \ldots, \frac{v_{d_{2}+1}-\frac{N+d_{2}}{d_{2}}}{\sqrt{h_{N+d_{2}-1}}}\right)\left(1+\mathcal{O}\left(\lambda^{-1}\right)\right),
\end{aligned}
$$

where $W$ is the matrix defined in eq. (3.1.10). Note that $W^{-1} \Omega W$ is just the permutation matrix (in the subblock). Q. E. D. 


\subsection{Stokes Matrices for the Fourier-Laplace transforms}

The fundamental solution of the system $\underline{D}_{1}$ is formed from $d_{2}$ Fourier-Laplace transforms $\underline{\Phi}^{(k)}(x), k=$ $1, \ldots d_{2}$, and one Hilbert-Fourier-Laplace transform $\underline{\Phi}^{(0)}(x)$. The asymptotic behavior of the $d_{2} \mathrm{FL}$ transforms is analyzed by means of the steepest descent method in each of the sectors $\mathcal{S}_{k}, k=0, \ldots 2 d_{2}+1$ (2.4.6), while the behavior of the (piecewise) analytic function $\underline{N}^{(0)}(x)$ is obtained (in each $\mathcal{D}_{\mu}$ ) from eq. (3.1.30). The computation is achieved by expressing the change of homology basis from the $\Gamma_{1}^{(y)}, \ldots, \Gamma_{d_{2}}^{(y)}$ contours to the steepest descent contours. In order to simplify the analysis of the Stokes matrices we point out that there is no essential loss of generality in assuming $V_{2}(y)=y^{d_{2}+1} \frac{1}{d_{2}+1}$. Indeed, we are concerned with just the homology classes of the SDC's, and as $x \rightarrow \infty$ the $d_{2}$ solutions of the equation $V_{2}^{\prime}(y)=x$ entering Def. 2.4 are distinct and asymptotic to the $d_{2}$ roots of $x$ (up to a nonzero factor). The particular choice of the leading coefficient is also essentially irrelevant. If we choose a different coefficient, we must just appropriately rotate by $\vartheta=\arg \left(v_{d_{2}+1}\right) /\left(d_{2}+1\right)$ counterclockwise in the pictures to follow, but without any essential difference. Therefore, we proceed with $v_{d_{2}+1}$ set equal to unity.

With these simplifications, the Stokes phenomenon can be studied directly on the integrals

$$
\int \mathrm{d} y \mathrm{e}^{-\frac{1}{\hbar}\left(\frac{y^{d+1}}{d+1}-x y\right)}=|x|^{\frac{1}{d}} \int \mathrm{d} z \exp \left[-\Lambda\left(\frac{z^{d+1}}{d+1}-\mathrm{e}^{i \alpha} z\right)\right],
$$

where

$$
\Lambda:=\frac{1}{\hbar}|x|^{\frac{d+1}{d}}, \quad \alpha:=\arg (x),
$$

and, in order to avoid too many subscripts in the formulae to follow, we have here, and for the remainder of this section, have set $d=d_{2}$. Disregarding the positive factor $|x|^{\frac{1}{d}}$, which is inessential for these considerations, the integrals in (3.2.2) can be written as

$$
\int \mathrm{d} z \exp \left[-\Lambda\left(\frac{z^{d+1}}{d+1}-\mathrm{e}^{i \alpha} z\right)\right]=\int \mathrm{d} s \mathrm{e}^{-\Lambda s} \frac{\mathrm{d} z}{\mathrm{~d} s},
$$

where $z=Z(s)$ is the $D+1$-valued inverse to

$$
s=S(z):=\frac{z^{d+1}}{d+1}-\mathrm{e}^{i \alpha} z, \quad z=Z(s) .
$$

It defines a $(d+1)$-fold covering of the $s$-plane branching around the points $(z, s)$ whose projection on the $s$-plane are the $d$ critical values

$$
s_{c r}^{(j)}=s_{c r}^{(j)}(\alpha)=-\frac{d}{d+1} \mathrm{e}^{i \frac{(d+1) \alpha}{d}} \omega^{j}, \quad \omega:=\mathrm{e}^{\frac{2 i \pi}{d}}, \quad j=0, \ldots, d-1 .
$$

In realizing this $d+1$-fold covering, we take the branch cuts on the $s$-plane to be the rays $\Im(s)=$ $\Im\left(s_{c r}^{(j)}\right)=$ const, extending to $\Re(s)=+\infty$. As $\Lambda \rightarrow+\infty$ the integrals 3.2.2 have leading asymptotic behavior that depends only on the critical values of the map $s(z)$ and on the homology class of the contour. 
We now return to the computation of the Stokes' lines. By the definition of the SDC's $\gamma_{k}$ (Def. 2.4, with $V_{2}(y)$ now taken as just $\left.y^{d+1} /(d+1)\right)$, their image in the $s$-plane consists of contours which come from $\Re(s)=+\infty$ on one side of the branch-cut (and on the appropriate sheet) and go back to $\Re(s)=+\infty$ on the other side of the branch-cut, on the same sheet. (For the SAC's, we choose cuts extending to $\Re(s)=-\infty$.) The cuts on the $s$-plane may overlap only for those values of $\alpha=\arg (x)$ for which the imaginary parts of two different critical values $s_{c r}^{(i)}(\alpha)$ and $s_{c r}^{(j)}(\alpha)$ coincide. A straightforward computation, with our simplifying assumptions on $V_{2}(y)$, yields the lines separating the sectors $\mathcal{S}_{k}$ to be those defined in eqs. (2.4.6) (with $d_{2}$ replaced by $d$ and $\vartheta=0$ ).

We also need the following.

Definition 3.1 For a given sector $\mathcal{S}$, of width $A<\pi$, centered around a ray $\arg (x)=\alpha_{0}$, the dual sector $\mathcal{S}^{\vee}$ is the sector centered around the ray $\arg (x)=-\alpha_{0}+\pi$, with width $\pi-A$.

For $x \rightarrow \infty$ in each sector the SDC's are constant integral linear combinations of the contours $\Gamma_{k}^{(y)}$ 's. When $x$ crosses the Stokes line between two adjacent sectors, the homology of the SDC's changes discontinuously. We denote the SDC's relative to the sector $\mathcal{S}_{k}$ by $\gamma_{j}^{(k)}, j=0 \ldots d-1$, and denote the column vector with these as entries $\vec{\gamma}^{(k)}$, and similarly, let $\vec{\Gamma}$ denote the column vector with entries $\left\{\Gamma_{j}^{(y)}\right\}_{j=1 \ldots d}$. Denoting the matrix of change of basis by $C_{k}$, we have

$$
\vec{\gamma}^{(k)}=C_{k} \vec{\Gamma}, C_{k} \in G L(d, \mathbb{Z}) .
$$

Our first objective is to compute these matrices $C_{k}$. For each fixed generic $\alpha$ (i.e. away from the Stokes' lines), we can construct a diagram (essentially a Hurwitz diagram) which describes the sheet structure of the inverse map $z=Z(s)$. We draw $d+1$ identical ordered $d$-gons each representing a copy of the $s$-plane and whose (labeled) vertices represent the projections of the $d$ critical values $s_{c r}^{(j)}$. Two vertices with the same label of two different $d$-gons are joined by a segment if the two sheets are glued together along a horizontal branch-cut originating at the corresponding critical value and going to $\Re(s)=+\infty$. Since all the branch-points of the inverse map are of order 2 , there are at most two sheets glued along each cut. Furthermore we give an orientation to the segments (represented by an arrow) with the understanding that this gives an orientation to the corresponding SDC. The convention is that an arrow going from sheet $j$ to sheet $k$ means that the SDC runs on sheet $j$ coming from $\Re(s)=+\infty$ below the cut and goes back above the same cut (or, what is "homologically" the same, the contour runs on sheet $k$ coming from $+\infty$ above the cut and returns to $+\infty$ below it).

The diagram can be uniquely associated to a matrix $Q_{k}$ of size $d \times(d+1)$, in which each row corresponds to a SDC and each column to a sheet. The matrix element $\left(Q_{k}\right)_{i j}$ is taken to equal:

-1 if the $i$-th SDC points to the $j$-th sheet,

1 if the $i$-th SDC originates on the $j$-th sheet, 

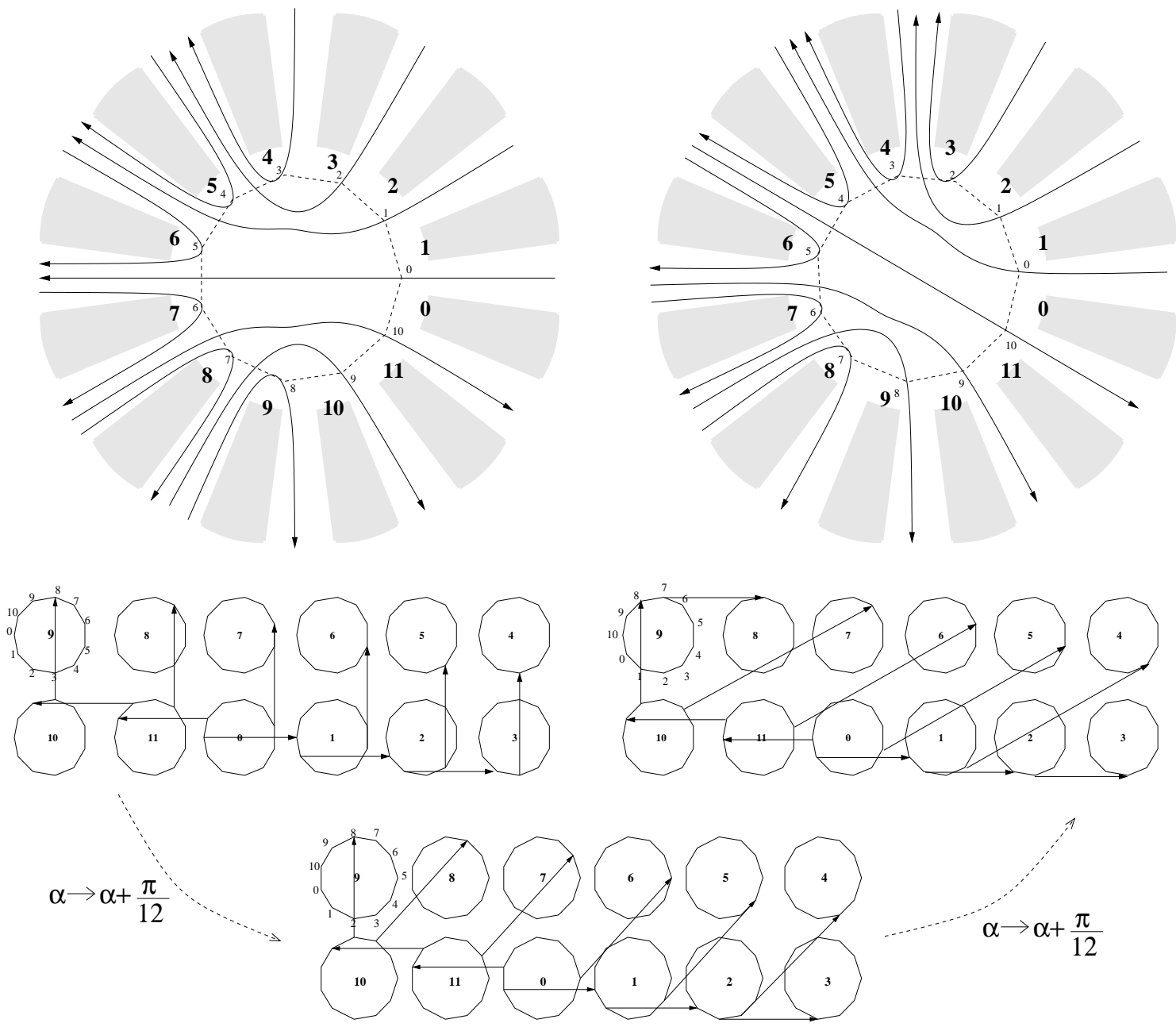

Figure 4: Example of the sheet structure for the case $d=11, \alpha=0$. The contours depicted in the figure are the SDC's in the $z$ plane. The second image represents the contours after incrementing $\alpha$ by $\pi / 6$, i.e., after crossing two Stokes lines. The labeling of the contours (smaller numbers in the top figures) is by the subscripts of the $\gamma_{k}$ 's and $\widetilde{\gamma}_{k}$ 's passing through the critical points $z_{c r}^{(k)}:=\mathrm{e}^{i \frac{\alpha}{d}} \omega^{k}$. This also corresponds to the numbering of the vertices of the endecagons representing the critical values $s_{c r}^{(k)}:=-\frac{d}{d+1} \mathrm{e}^{\frac{d+1}{d} \alpha} \omega^{k}$. The different sheets of the $s$-plane are mapped onto the connected components in which the $z$-plane is cut by the SDC's. These are the images in the $z$-plane of the cuts in the $s$-plane. The labeling of each sheet is given by the boldface numbers and corresponds to the labels inside the endecagons appearing in the extended Hurwitz diagrams below the corresponding $z$-planes. The intermediate Hurwitz diagram represents the gluing of the sheets after crossing the intermediate Stokes line, i.e., after incrementing $\alpha$ by $\pi / 12$.

0 otherwise.

Hence each row of the matrix has exactly one +1 and one -1 entry. 
In Fig. 1 , we indicate, for the case $d=11, \alpha=0$, the SDS contours and sheet structure represented by the extended Hurwitz diagram. The matrix corresponding to this diagram is:

$$
\begin{aligned}
& \begin{array}{llllllllllll}
0 & 1 & 2 & 3 & 4 & 5 & 6 & 7 & 8 & 9 & 10 & 11
\end{array}
\end{aligned}
$$

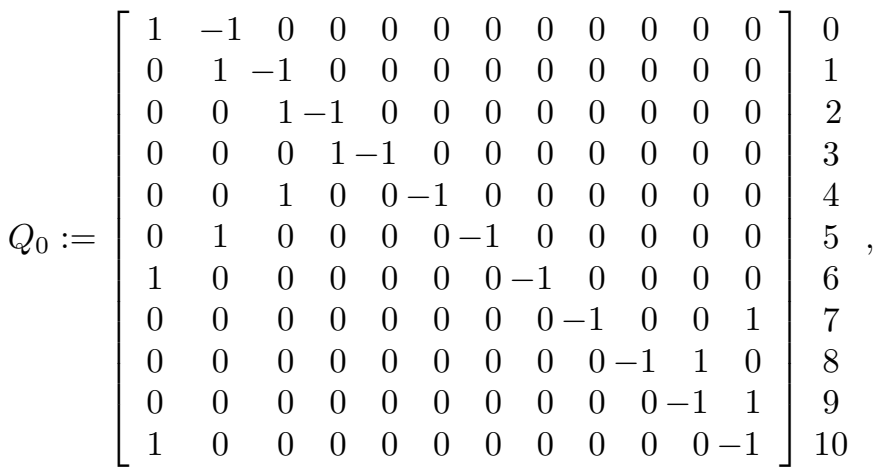

where the boldface numbers labeling the columns are the corresponding labels for the sheets, while the numbers labeling the rows are the labels of the SDC's. Since $\alpha=\arg (x)$ ranges within a fixed sector $\mathcal{S}_{k}$, the diagram does not change topology and the corresponding matrix $Q_{k}$ remains unchanged.

We can now describe how a given diagram changes when $\alpha=\arg (x)$ crosses the line between two adjacent sectors $\mathcal{S}_{k}$ and $\mathcal{S}_{k+1}$ (counterclockwise). These lines correspond precisely to the values of $\alpha$ for which two distinct critical values have the same imaginary parts (so that the cuts may overlap if they are on the same sheet). We leave to the reader to check that these lines are precisely the boundaries of the Stokes sectors $\mathcal{S}_{k}$ defined in eq. (2.4.6). As $\alpha$ increases by $\pi /(d+1)$ from $\mathcal{S}_{k}$ to $\mathcal{S}_{k+1}$. the $d$-gons rotate by $\pi / d$. In this process the connections between the sheets change according to the following rule: if the branch-point $P_{j}$ on sheet $r$ crosses the cut originating from a different branch-point $P_{h}$ on the same sheet (on the left of $P_{j}$ on sheet $r$, and hence $P_{j}$ crosses the cut from below as it moves upwards), then the $P_{j}$ (and its cut) jumps to the sheet $s$ which is glued to sheet $r$ along the cut originating at $P_{h}$ (see Fig. 5). Diagrammatically, the tip (or the tail) of the corresponding arrow moves from one $d$-gon to another one connected along the vertex $h$. In terms of the matrix $Q_{k}$, the $j$-th row reflects along the hyperplane orthogonal to the $h$-th row. The homology class of the SDC $\gamma_{h}^{(k)}$ will then change because the branch-cut attached to $P_{j}$ which "emerges" from the branch cut attached to $P_{h}$ "extracts" a contribution proportional to $\gamma_{j}^{(k)}$. The proportionality factor is \pm 1 depending on the relative orientations. The corresponding SDC's $\gamma_{j}^{(k)}$ and $\gamma_{h}^{(k)}$ are related to the SDC's $\gamma_{j}^{(k+1)}$ and $\gamma_{h}^{(k+1)}$ by the relations

$$
\begin{aligned}
& \gamma_{j}^{(k+1)}=\gamma_{j}^{(k)} \\
& \gamma_{h}^{(k+1)}=\gamma_{h}^{(k)}+\epsilon_{h j} \gamma_{j}^{(k)}
\end{aligned}
$$

where the incidence number $\epsilon_{h j}=\epsilon_{j h}$ is 1 if the SDC's $\gamma_{j}^{(k)}, \gamma_{h}^{(k)}$ have the opposite orientation and -1 if they have the same orientation. Alternatively, the incidence number is just the (standard) inner product of the corresponding rows $h, j$ of the matrix $Q_{k}$. 


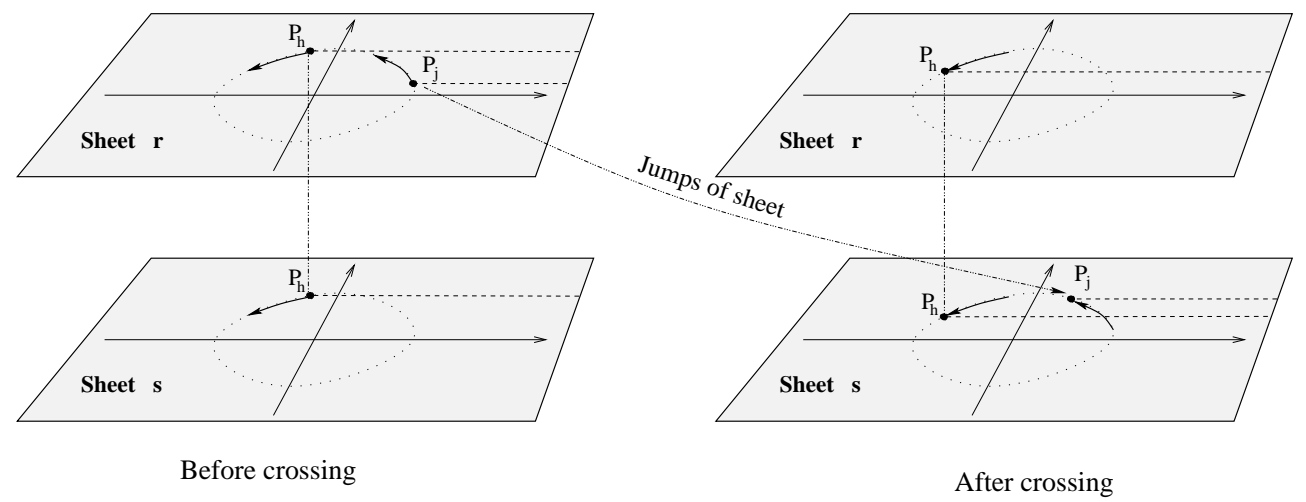

Figure 5: The crossing of two critical values and the corresponding cuts.

We denote by $M_{k}$ the $d \times d$ matrix which expresses the changes in the SDC's described by eq. (3.2.8) through the relation

$$
\vec{\gamma}^{(k+1)}=M_{k} \vec{\gamma}^{(k)}
$$

These will be seen presently to be precisely the Stokes matrices for the passage between these sectors. One can check that the matrices $Q_{k}$ and $Q_{k+1}$ are related by

$$
Q_{k+1}=M_{k}^{t-1} Q_{k}
$$

Therefore we can reconstruct the matrices $M_{k}$ once we have an initial diagram representing the sheet structure.

Before constructing the initial diagram, we note that when $\alpha$ increases by $2 \pi /(d+1)$ (i.e. when we cross two Stokes lines), the steepest descent (unoriented) contours are like the original ones, but rotated by the same amount clockwise. That is, the unoriented diagram (i.e. forgetting the orientation of the SDC's) is the same, up to cyclically permuting the labels of the $(d+1)$ sheets and the $d$ cuts. Note also that the critical points rotate by $\frac{2 \pi}{d(d+1)}$ and the critical values by $\frac{2 \pi}{d}$ counterclockwise. Indeed we have

$$
s\left(z ; \alpha+\frac{2 \pi}{d+1}\right)=\frac{z^{d+1}}{d+1}-\mathrm{e}^{i \alpha} \mathrm{e}^{i \frac{2 \pi}{d+1}} z=s\left(\mathrm{e}^{i \frac{2 \pi}{d+1}} z ; \alpha\right) .
$$

As for the orientation of the SDC's relative to the new labeling, the $d$-th SDC passing through $z_{c r}^{(d-1)}$ reverses its orientation relative to the (oriented) SDC's obtained by just rotating the initial SDC's (see Figure (1). This implies that the matrices $Q_{k}$ representing the diagrams in the various sectors and the Stokes' matrices $M_{k}$ satisfy the recursion relations

$$
Q_{k+2}=p^{-1} Q_{k} \mathfrak{S}_{d+1}, \quad M_{k+2}=p \cdot M_{k} \cdot p^{-1}
$$




$$
\mathfrak{S}_{d+1}=\left[\begin{array}{ccccc}
0 & 0 & \cdots & 0 & 1 \\
1 & 0 & \cdots & 0 & 0 \\
0 & 1 & \cdots & 0 & 0 \\
0 & 0 & \ddots & 0 & \vdots \\
0 & 0 & \cdots & 1 & 0
\end{array}\right] \in \mathrm{GL}(d+1, \mathbb{Z}), \quad p:=\left[\begin{array}{ccccc}
0 & 0 & \cdots & 0 & -1 \\
1 & 0 & \cdots & 0 & 0 \\
0 & 1 & \cdots & 0 & 0 \\
0 & 0 & \ddots & 0 & 0 \\
0 & 0 & \cdots & 1 & 0
\end{array}\right] \in \mathrm{GL}(d, \mathbb{Z})
$$

It is therefore only necessary to compute $Q_{0}, Q_{1}$ and $M_{0}, M_{1}$.

Notice also that the wedge contours $\Gamma_{j}^{(y)}, j=0, \ldots, d$ are in $1-1$ correspondence with the sheets of the map $Z(s)$. Indeed, each connected component to the right of the wedge contours contains only one sector $S_{2 k+1}^{(y)}$ in the $z$-plane which corresponds to the sector $\Re(s)<0$ in the $s$-plane. Thus, the same argument used to arrive at the recursion (3.2.12) proves that

$$
\begin{aligned}
C_{k+2} & =p C_{k} P \\
P & :=\left[\begin{array}{rrrrr}
-1 & -1 & \cdots & -1 & -1 \\
1 & 0 & \cdots & 0 & 0 \\
0 & 1 & \cdots & 0 & 0 \\
0 & 0 & \ddots & 0 & 0 \\
0 & 0 & \cdots & 1 & 0
\end{array}\right] \in \mathrm{GL}(d, \mathbb{Z}),
\end{aligned}
$$

where both the matrix $\mathfrak{S}_{d+1}$ in eq. (3.2.12) and $P$ in eq. (3.2.13) generate a representation of the cyclic group $\mathbb{Z}_{d+1}$ (although $P$ is of size $d \times d$ ). The matrix $P$ is just the generator of $\mathbb{Z}_{d+1}$ on the "wedge" contours; i.e. with the additional constraint $\sum_{i=0}^{d} \Gamma_{i}^{(y)}=0$.

We now describe how to derive the initial diagram - for example, how Figure 1 is obtained. We start by noting that the "wedge" contours $\Gamma_{k}^{(y)}$ enclose the sector $\mathcal{S}_{2 k-1}$ of width $\pi /(d+1)$. It is to see that the corresponding integrals are (more than) exponentially decreasing in the dual sector, since

$$
\left|\int_{\Gamma_{k}^{(y)}} \mathrm{e}^{-\frac{1}{\hbar}\left(y^{d+1} /(d+1)-x y\right)} \mathrm{d} y\right| \leq \exp (|x| M) \int_{\Gamma_{k}^{(y)}}\left|\mathrm{e}^{-\frac{1}{\hbar} y^{d+1} /(d+1)}\right| \mathrm{d} y,
$$

where $M$ is the supremum of $\frac{1}{|x|} \Re(x y)$ as $y$ goes along $\Gamma_{k}^{(y)}$. Now the contour $\Gamma_{k}^{(y)}$ can be deformed so as to approach the sector $\mathcal{S}_{2 k-1}^{(y)}$ as closely as we wish. Then the constant $M$ is finite and negative if $x$ lies within the dual sector $\mathcal{S}_{k}^{(y)^{\mathrm{v}}}$.

We now consider the case of odd $d$, leaving the easy generalization to even $d$ 's to the reader. (The only difference is that for odd $d, \alpha=\arg (x)=0$ is not a Stokes' line. To study the case even $d$ case, one should choose a convenient initial value of $\alpha$ (e.g. $\alpha=\epsilon \ll 1$ or $\alpha=\pi / 2(d+1)$, which corresponds to an anti-Stokes line).

Let us start with the (non-Stokes) value $\alpha=0$ and focus on any of the SDC's attached to a critical value lying in the right $s$-plane for this value of $\alpha$ (remembering that $d$ is assumed to be odd). Since the real part of such a critical value is positive, the corresponding integrals decrease as $\exp \left(-\frac{d}{d+1} \omega^{j} \Lambda\right)$, $\left(\Lambda:=\frac{1}{\hbar}|x|^{\frac{d+1}{d}}\right)$ i.e., they are (more than) exponentially suppressed on the line $x \in \mathbb{R}_{+}$. As we increase 
$\alpha$, the $d$-gons rotate by $\frac{d+1}{d} \alpha$ counterclockwise. It should be clear (from the previous description of the change of homology of the SDC's) that the SDC's attached to such critical values do not change homology class while they remain in the right half of the $s$-plane. This is so because there are no branch points to their right which can pass through the branch-cut through the given saddle point (which extends to the right) as $\alpha$ is increased. Therefore the corresponding integrals are exponentially suppressed as long as $\alpha$ ranges in a corresponding sector of width $\frac{d}{d+1} \pi=\pi-\frac{1}{d+1} \pi$. This is precisely the width of the dual sector to a sector of width $\frac{\pi}{d+1}$; i.e., the width of any of the $S_{2 k+1}^{(y)}$ 's. By careful inspection of the anti-Stokes lines for these integrals 9 , which correspond to the value of $\alpha$ for which the corresponding critical value is real and positive, one concludes that they coincide with an appropriate $\Gamma_{k}^{(y)}$ in the left half of the $z$-plane. This argument proves that all "wedge" contours $\Gamma_{k}^{(y)}$ lying in the left plane are homological to SDC's. (These are the SDC's in the left $z$-plane in Figure 4 .)

We then take the first critical value lying in the left half of the $s$-plane (in Figure 4 , the SDC number 9 ). As we increase $\alpha$ so as to move this critical value to the right half of the $s$-plane, the corresponding SDC can acquire a contribution only from the first SDC in the left half-plane (number 8 in our example). As a consequence the corresponding integral is exponentially suppressed in a sector of width $\pi-2 \frac{\pi}{d+1}$. Considering its anti-Stokes line and its linear independence from the previously identified SDC's, we conclude that it must enclose two odd-numbered sectors $S_{2 k+1}$ and $S_{2 k+3}$ (in our example this is contour 9). Proceeding this way we can easily identify the homology classes of all SDC's for $\alpha=0$.

The labeling of the sheets is largely arbitrary. (The choice we have made in the fig. 4 is just for "aesthetic" reasons). The fixed basis of contours $\Gamma_{i}^{(y)}, i=1, \ldots d$ and the bases $\gamma_{j}^{(k)}, j=0, \ldots d-1 k=$ $0, \ldots 2 d+1$ are related by

$$
\begin{aligned}
& \vec{\gamma}^{(k)}=C_{k} \vec{\Gamma}=M_{k-1} \cdots M_{0} C_{0} \vec{\Gamma} \\
& \vec{\gamma}^{(0)}=C_{0} \vec{\Gamma}
\end{aligned}
$$

Therefore only the matrices $M_{k}$ and the first change of basis matrix $C_{0}$ are needed. The matrix $C_{0}$ can easily be constructed from the initial sheet structure. For our present purposes it is not actually necessary to have its general form, since the objects of primary interest are the Stokes matrices, which will be seen to be just the $M_{k}$ 's. But to illustrate by example the form that $C_{0}$ takes, within the basis

\footnotetext{
${ }^{6}$ The anti-Stokes' line for exponential integrals of the type of Def. 2.4 are the lines along which the integrals are most rapidly decreasing as $|x| \rightarrow \infty$.
} 
we have chosen, for the case $d=11$, it is:

$$
C_{0}:=\left[\begin{array}{lllllllllll}
1 & 1 & 1 & 1 & 1 & 1 & 0 & 0 & 0 & 0 & 0 \\
0 & 1 & 1 & 1 & 1 & 0 & 0 & 0 & 0 & 0 & 0 \\
0 & 0 & 1 & 1 & 0 & 0 & 0 & 0 & 0 & 0 & 0 \\
0 & 0 & 0 & 1 & 0 & 0 & 0 & 0 & 0 & 0 & 0 \\
0 & 0 & 0 & 0 & 1 & 0 & 0 & 0 & 0 & 0 & 0 \\
0 & 0 & 0 & 0 & 0 & 1 & 0 & 0 & 0 & 0 & 0 \\
0 & 0 & 0 & 0 & 0 & 0 & 1 & 0 & 0 & 0 & 0 \\
0 & 0 & 0 & 0 & 0 & 0 & 0 & 1 & 0 & 0 & 0 \\
0 & 0 & 0 & 0 & 0 & 0 & 0 & 0 & 1 & 0 & 0 \\
0 & 0 & 0 & 0 & 0 & 0 & 0 & 0 & 1 & 1 & 0 \\
0 & 0 & 0 & 0 & 0 & 0 & 0 & 1 & 1 & 1 & 1
\end{array}\right]
$$

It is not difficult to give an explicit description of the matrices $Q_{0}, Q_{1}$ and $M_{0}, M_{1}$ but, for the sake of brevity, we will not give it here. It consists of a lengthy but straightforward calculation, which leads to the matrices in Table 1 (listed for the cases $d=2, \ldots, 11$ ), where one can clearly extrapolate to the correct rule.

We now turn our attention to the Stokes matrices. Consider the subblock of the fundamental system corresponding to solutions of the $\underline{D}_{1}$ ODE given by the Fourier-Laplace transforms. If we denote by $Y(x)$ the $d_{2} \times d_{2}$ such block whose rows are the integrals of $\stackrel{N}{\varphi}(y)$ on the contours $\Gamma_{k}^{(y)}$, and by $Y_{k}(x)$ the analogous matrix obtained by integrating over the SDC's, we have

$$
Y_{k}(x)=C_{k} Y(x)
$$

The Stokes matrices are then given by

$$
S_{k}:=Y_{k+1} Y_{k}^{-1}=C_{k+1} C_{k}^{-1}=M_{k} .
$$

These are just the matrices expressing the relative change of homology basis of the SDC's corresponding to two consecutive Stokes' sectors.

In order to complete the description of the Riemann-Hilbert problem, we need to also consider the extra solution given by a Hilbert-Fourier-Laplace transform. In doing so, we extend the previously computed Stokes matrices $M_{k}$ to the full fundamental system of $d_{2}+1$ solutions by means of

$$
\widehat{M}_{k}:=\left[\begin{array}{c|c}
1 & 0 \\
\hline 0 & M_{k}
\end{array}\right] .
$$

Summarizing the whole discussion, we have proved the following theorem.

Theorem 3.1 (Riemann-Hilbert Problem) There exists a fundamental system of solutions, $\underline{N}(x)$ which is analytic where defined, with the properties: 


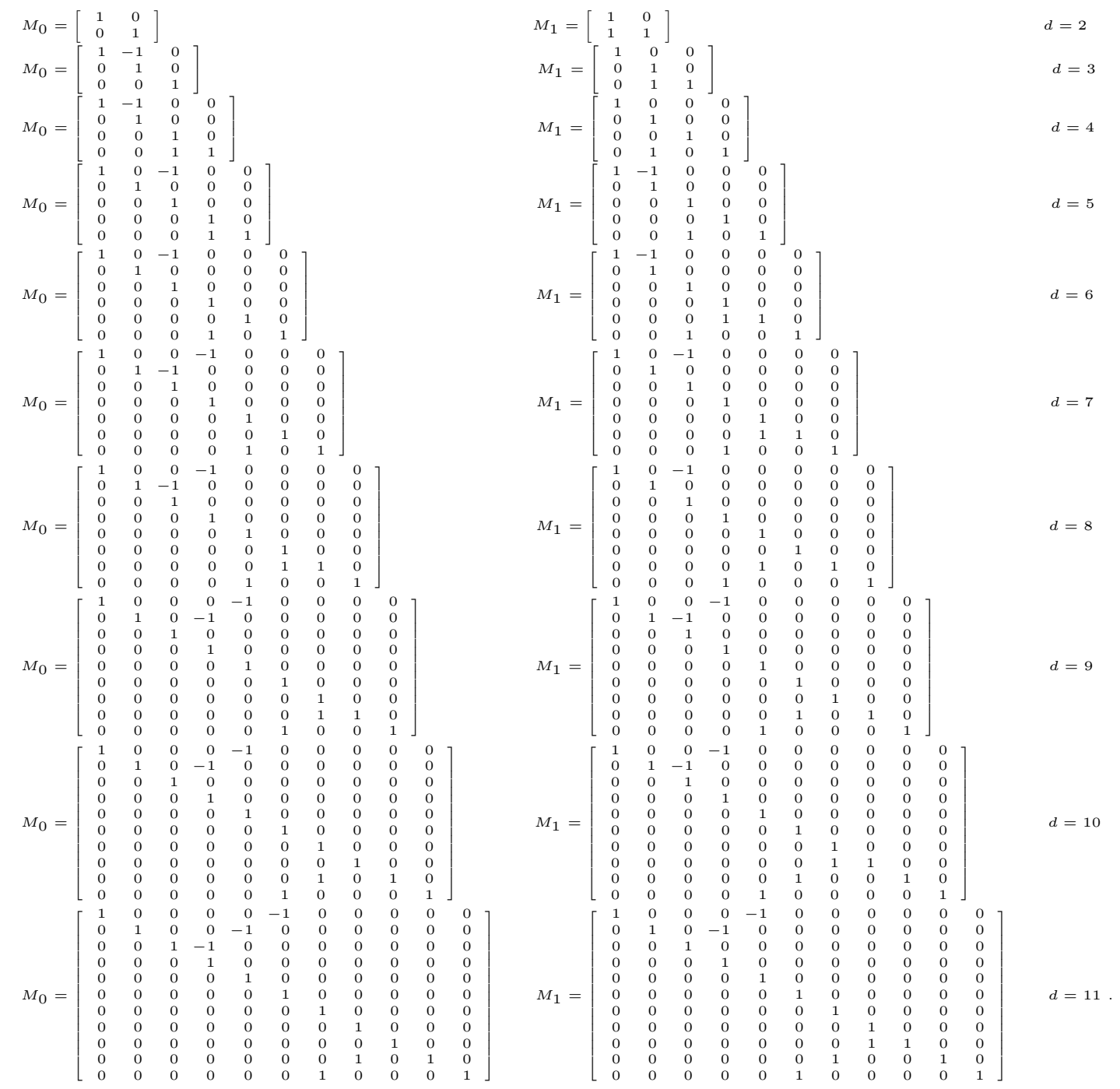

Table 1: The first two Stokes matrices for degrees $d_{2}=2 \ldots 11$. The remaining ones are obtained using eq. (3.2.12). 
1. In each of the sectors around $x=\infty$ defined by removing the lines $L_{\mu}, \mu=0, \ldots d_{1}$ and $\mathcal{R}_{k}$, $k=0, \ldots, 2 d_{2}+1, \stackrel{N}{\mathbf{\Phi}}(x)$ exists, is analytic and invertible, and it can be normalized on the left by a constant matrix to have the same asymptotic behavior as $\underline{\boldsymbol{\Phi}}_{\text {form }}(x)$ defined in Proposition 3.1 .

2. Crossing the lines $L_{\mu}$, the corresponding Stokes matrix is given by $G_{\mu}$ as defined in eq. (3.1.0).

3. Crossing the lines $\mathcal{R}_{k}$ the corresponding Stokes matrix is given by $\widehat{M}_{k}$ in eq. (3.2.20), constructed according to the algorithm described in section 3.9 .

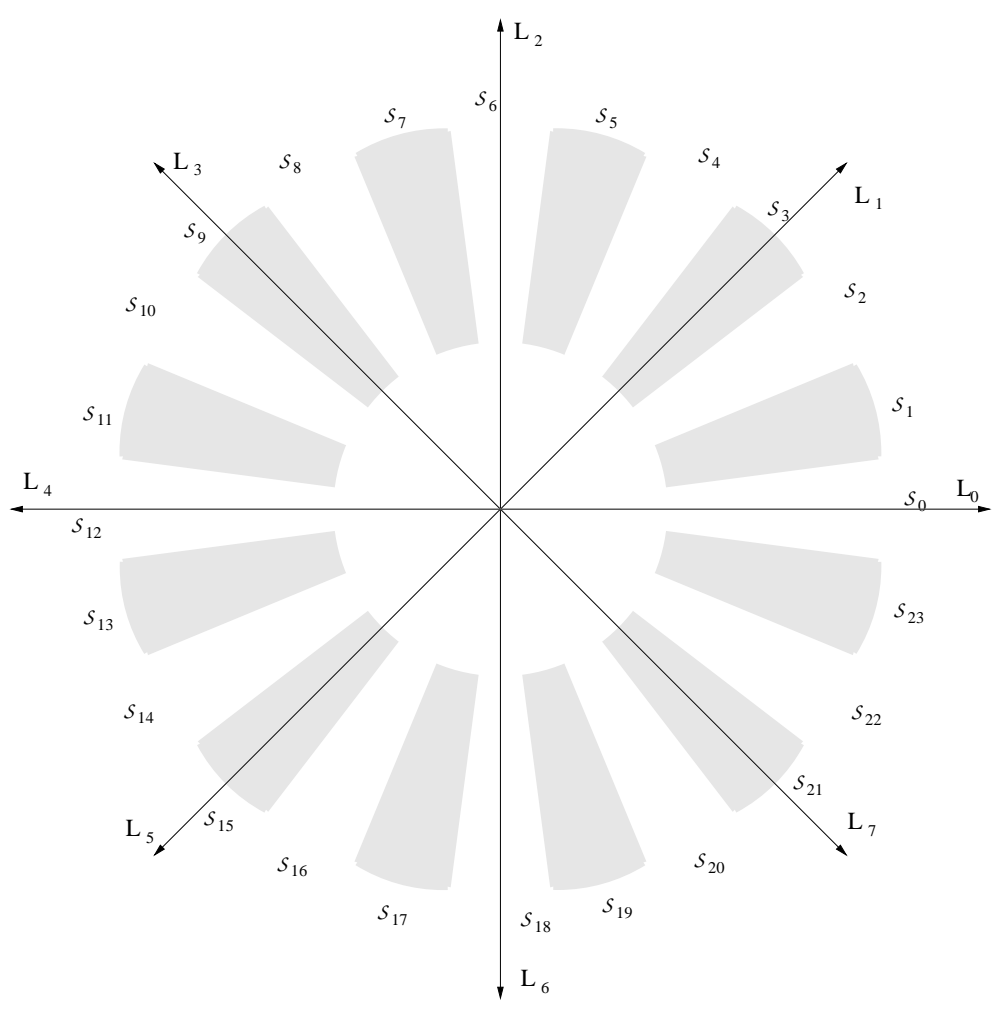

Figure 6: Example of the structure of Stokes sectors and discontinuity lines $L_{\mu}$ (which can equivalently be viewed as Stokes lines) in the $x$-plane for the case $d_{1}=7$ and $d_{2}=11$ and both leading coefficients of the potentials real and positive.

For an example of all Stokes' sectors and lines see Fig. 6] and for examples of explicit Stokes' matrices $M_{0}, M_{1}$ from which all the others are computed easily, see Table 1 .

We conclude this section with the remark that in the formulation of Theorem 3.1 the lines $L_{\mu}$ were viewed as Stokes' lines. However, we could alternatively have formulated an equivalent Riemann-Hilbert problem, in which the lines $L_{\mu}$ define discontinuities, according to eq. 3.1.6), by retaining a fixed choice of the contours of integration along the boundaries of the wedge sectors, thereby giving rise to genuine jump discontinuities across the boundaries. 


\section{Summary and comments on large $N$ asymptotics in multi- matrix models}

A complete formulation of the Riemann-Hilbert problem characterizing fundamental systems of solutions to the differential - recursion equations satisfied by biorthogonal polynomials associated to 2-matrix models with polynomial potentials is provided in Theorem 3.1. The approach derived here can also be extended quite straightforwardly to the case of biorthogonal polynomials associated to a finite chain of coupled matrices with polynomial potentials, following the lines indicated in the appendix of [2].

The Riemann-Hilbert data, consisting essentially of the Stokes matrices at $\infty$, are independent of both the integer parameter $N$ corresponding to the matrix size and the deformation parameters determining the potentials; that is, the fundamental solutions constructed are solutions simultaneously of a differential-difference generalized isomonodromic deformation problem. This provides the first main step towards a rigorous analysis of the $N \rightarrow \infty, \hbar N=\mathcal{O}(1)$ limit of the partition function and the Fredholm kernels determining the spectral statistics of coupled random matrices (essential, e.g., to the question of universality in 2-matrix models). Such an analysis should follow similar lines to those previously successfully applied to ordinary orthogonal polynomials in the 1-matrix case [6, 17, 19, 20, 10, 11]. The main difference in the 2-(or more) matrix case is that in the double-scaling limit the functional dependence of the free energy on the eigenvalue distributions is not as explicit as in the 1-matrix models [23, 18]. It is also clear that the hyperelliptic spectral curve that arises in the solution of the one-matrix model must be replaced by a more general algebraic curve, which arises naturally in the spectral duality of the spectral curves of [2] (cf. 14).

In order determine the large $N$ asymptotics with the help of the data defining the Riemann-Hilbert problem, one should begin with an ansatz that can be checked a posteriori against the given case. In the 1-matrix case [10, 11], this was provided by means of hyperelliptic $\Theta$-functions. The physical heuristics and the basic tools for generating such ansatz were also given in 17, 14, 23, 18. Much of of these heuristics can be extended to the 2-matrix case [4], and this will be the subject of a subsequent work [1].

\section{A Appendix: Convergent integral representations of $\underset{\infty}{\Psi}(x)$}

In this appendix, we indicate how to overcome the problem in the formal definition of eq. 2.3.15), illustrated through an example. The idea follows that used in 21] for the case of cubic potentials?.

As discussed in section $\underset{\infty}{\Psi^{(0)}}(x)$, a natural approach to finding solutions of eq. 2.2.7) would be to take the inverse Fourier-Laplace transform of the function

$$
\frac{\Psi}{\infty}^{(0)}(y):=\mathrm{e}^{\frac{1}{\hbar} V_{2}(y)} \int_{\varkappa \Gamma} \mathrm{d} x \mathrm{~d} t \frac{\mathrm{e}^{-\frac{1}{\hbar}\left(V_{1}(x)-x t\right)}}{y-t} \Psi^{t}(x),
$$

\footnotetext{
${ }^{7}$ We wish to thank A. Kapaev for pointing out the problem of divergent integrals appearing in an earlier version of this paper, and for helpful discussions. The approach outlined here, for potentials of arbitrary degrees, will be the subject of further work, and the results detailed in a subsequent, joint publication
} 
along the anti-wedge contours $\widetilde{\Gamma}_{k}^{(y)}$. Eq. (A-1) defines a piecewise analytic function in the domains

$$
\mathbb{C}_{y} \backslash \bigcup_{k=1}^{d_{2}} \Gamma_{k}^{(y)}=\bigcup_{\nu=0}^{d_{2}} \mathcal{D}_{\nu}
$$

with jumps across the $\Gamma_{k}^{(y)}$ given by

$$
\underline{\Psi}_{\infty}^{(0)}\left(y_{+}\right)-\underline{\Psi}_{\infty}^{(0)}\left(y_{-}\right)=2 i \pi \sum_{j=1}^{d_{1}} \varkappa^{j, k} \underline{\Psi}^{(j)}(y), \quad y \in \Gamma_{k}^{(y)} .
$$

However, either retaining this definition or modifying it through analytic continuation leads to a divergent integral when the Fourier-Laplace transform is applied. In order to resolve this difficulty, the idea is to consider a suitable contour approaching $y=\infty$ within the odd-numbered sectors $\mathcal{S}_{y}^{(2 k+1)}$ and define a locally analytic function in a neighborhood of this contour so as to render the Fourier-Laplace transform convergent.

A general approach resolving this problem is not needed for the results presented in this paper since, as pointed out, the Riemann-Hilbert problem for $\underset{N}{\mathbf{\Psi}}(x)$ is more simply obtained by means of duality (Remark 3.1). Therefore, we just give an indication of how to proceed by means of an example.

\section{A.1 An Example: $d_{1}=7, d_{2}=4$}

The example we consider consists of two potentials $V_{1}(x)$ and $V_{2}(y)$ of degrees 8 and 5 respectively, both with positive real leading coefficients. We refer to Fig. 7 as the "the figure" throughout this section. The $\mathcal{S}_{y}^{(k)}$ sectors are the light-shaded ones while the $\mathcal{S}_{x}^{(j)}$ are the darker-shaded ones. Fix an asymptotic ray $\ell_{\mathcal{L}}$ with $\arg (y)=\alpha_{0}(=\pi)$ within an odd-numbered sector, in our example $\mathcal{S}_{y}^{(5)}$, and an $\epsilon$ sector around it. Let $\mathcal{L}$ be the dual sector, i.e., the sector centered around $\pi-\alpha_{0}(=0)$ with width $\pi-\epsilon$. Any of the functions $\frac{\Psi}{\infty}(j)(y)$ whose wedge contours $\Gamma_{j}^{(x)}$ lie in the sector $\mathcal{L}$ decay faster than any exponential $\mathrm{e}^{-M y}$ on the given ray $\ell_{\mathcal{L}}$. These are the sectors $\mathcal{S}_{x}^{(1,3,13,15)}$ in Fig. 7. Fix another ray $\ell_{\mathcal{R}}$ within a different odd-numbered sector $\mathcal{S}_{y}^{(2 k+1)}$ in such a way that the dual sector to the $\epsilon$ sector around $\ell_{\mathcal{R}}$ (denoted by $\mathcal{R})$ contains all but one of the remaining sectors $\mathcal{S}_{x}^{(2 j+1)}$. In the choice shown in the figure $\ell_{\mathcal{R}}$ lies in $\mathcal{S}_{9}^{(y)}$ and the sector $\mathcal{R}$ contains the sectors $\mathcal{S}_{x}^{(7,9,11)}$. Finally, fix a contour $\widetilde{\Gamma}$ which goes off to infinity asymptotic to the two rays $\ell_{\mathcal{L}}, \ell_{\mathcal{R}}$ (as in figure).

With these choices, redefine the duality pairing according to the following rule:

(i) In the $x$-plane use as basis only the contours $\Gamma_{j}^{(x)}$ which fall within one or the other of the sectors $\mathcal{L}, \mathcal{R}$. In our example this leaves out contour $\Gamma_{3}^{(x)}$, which has not been drawn in the figure.

(ii) In the $y$-plane use a basis in which the contours $\widetilde{\Gamma}$ intersect only one of the $\Gamma_{k}^{(y)}$ s. In our example this would leave out either $\Gamma_{3}^{(y)}$ or $\Gamma_{0}^{(y)}$ and we have chosen the latter. 


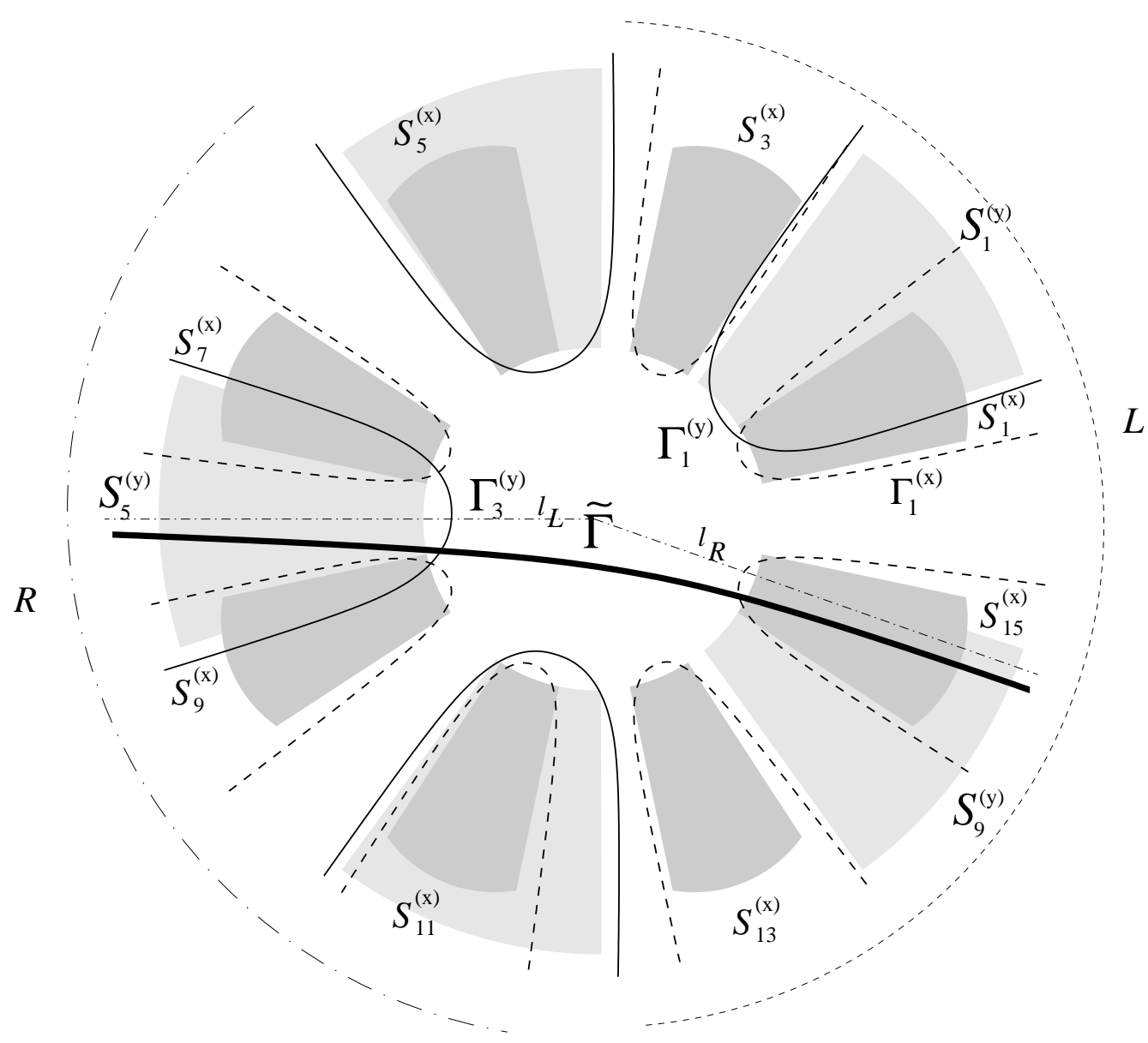

Figure 7: The figure illustrating the definition of $\Theta_{\infty}^{(\text {Left,Right })}(y)$. The contour $\widetilde{\Gamma}$ must approach $\infty$ within the sectors $\mathcal{S}_{2 k+1}^{(y)}$ 's (lighter shaded) and asymptotic to the two rays $\ell_{\mathcal{L}}, \ell_{\mathcal{R}}$. The terms added to $\underline{\Psi}_{\infty}^{(0)}$ in the definition of $\Theta_{\infty}^{(\text {Left })}\left(_{\infty}^{\left(\Theta^{(R i g h t)}\right)}\right)$ must correspond to sectors $\mathcal{S}_{2 j+1}^{(x)}$ which fall in the dual sector $\mathcal{L}(\mathcal{R})$ indicated by the dashed (dash-dotted) arc.

Note that we can accomplish an equivalent duality pairing using such bases by exploiting the homological equations

$$
\sum_{j=0}^{d_{1}} \Gamma_{j}^{(x)}=\sum_{k=0}^{d_{2}} \Gamma_{k}^{(y)}=0 .
$$

Indeed, using the homological equation (A-1-1), one can avoid one contour and redefine the $\varkappa^{\prime}$ s:

$$
\varkappa \Gamma=\sum_{i \neq 0, j \neq 0} \varkappa^{i, j} \Gamma_{i}^{(x)} \times \Gamma_{j}^{(y)}=\sum_{i \neq 3, j \neq 0} \tilde{\varkappa}^{i, j} \Gamma_{i}^{(x)} \times \Gamma_{j}^{(y)},
$$


where $\tilde{\varkappa}^{i, j}$ in this example would be

$$
\tilde{\varkappa}^{i, j}:=\varkappa^{i, j}-\varkappa^{3, j}, \quad i \neq 3,0 ; \quad \tilde{\varkappa}^{0, j}:=-\varkappa^{3, j} .
$$

Now define the wave-vector

$$
{\underset{\infty}{\Theta}}^{\text {Left }}(y):=\underline{\Psi}_{\infty}^{(0), t}(y)-2 i \pi \sum_{i=0,1,2,7} \tilde{\varkappa}^{i, 3} \frac{\Psi}{\infty}^{(i), t}(y)=: \frac{\Psi}{\infty}^{(0), t}(y)-2 i \pi \sum_{i \in I_{\mathcal{L}}} \tilde{\varkappa}^{i, 3} \frac{\Psi}{\infty}^{(i), t}(y),
$$

for $y$ belonging to the part of $\widetilde{\Gamma}$ inside contour $\Gamma_{3}^{(y)}$. (Note the transposition of row vectors to column vectors.) In eq. (A-1-4) in general $I_{\mathcal{L}}$ would be the set of indices $j$ corresponding to the contours $\Gamma_{j}^{(x)}$ contained in sector $\mathcal{L}$. Note that this function decays on the ray $\ell_{\mathcal{L}}$ because $\ell_{\mathcal{L}}$ is in the dual sector of all the $\underset{\infty}{\Psi^{(j)}}$ entering eq. (A-1-4). Moreover the piecewise analytic function $\underset{\infty}{\Psi^{(0)}}$ decays exponentially in each sector $\mathcal{S}_{k}^{(y)}$.

As we cross the contour $\Gamma_{3}^{(y)}$ along $\widetilde{\Gamma}$, by virtue of the jump condition $(\widehat{A-3})$, the function $\Theta_{\infty}^{\text {Left }}(y)$ is analytically continued to the function

$$
\underset{\infty}{\Theta^{R i g h t}}(y):=\frac{\Psi}{\infty}^{(0), t}(y)+2 i \pi \sum_{j \notin L} \tilde{\varkappa}^{j, 3} \frac{\Psi}{\infty}^{(j), t}(y):=\frac{\Psi}{\infty}^{(0), t}(y)+2 i \pi \sum_{j=4,5,6} \tilde{\varkappa}^{j, 3} \frac{\Psi}{\infty}^{(j), t}(y)
$$

which, due to the choices made above, also decays along the asymptotic direction $\ell_{\mathcal{R}}$. The two functions ${\underset{\infty}{\boldsymbol{\Theta}}}^{\text {Left,Right }}(y)$ define together a function $\underset{\infty}{\boldsymbol{\Theta}^{\widetilde{\Gamma}}}(y)$ which is (locally) analytic in a neighborhood of the contour $\widetilde{\Gamma}$ and decays sufficiently fast in either direction to allow us to take an inverse Fourier-Laplace transform using

$$
\Psi_{\infty}^{(\widetilde{\Gamma})}(x):=\int_{\widetilde{\Gamma}} \mathrm{d} y \mathrm{e}^{-\frac{x y}{\hbar}}{\underset{\infty}{(\widetilde{\Gamma})}}^{(\widetilde{S})} .
$$

This wave-vector is a solution of eqs. (2.2.8), 2.2.40) and (2.2.41) as we now show. The equations

$$
\begin{aligned}
& y \underset{\infty}{\Theta^{(\widetilde{\Gamma})}}(y)=P \underset{\infty}{\Theta^{(\widetilde{\Gamma})}}(y)+\left[\sqrt{h_{0}} \mathrm{e}^{\frac{1}{\hbar} V_{2}(y)}, 0, \ldots\right]^{t} \\
& \hbar \partial_{y} \underset{\infty}{\Theta^{(\widetilde{\Gamma})}}(y)=Q \underset{\infty}{\boldsymbol{\Theta}^{(\widetilde{\Gamma})}}(y)+W_{2}(y)\left[\sqrt{h_{0}} \mathrm{e}^{\frac{1}{\hbar} V_{2}(y)}, 0, \ldots\right]^{t} \\
& \hbar \partial_{u_{K}}{\underset{\infty}{\Theta}}^{(\widetilde{\Gamma})}(y)=\mathrm{U}^{K}{\underset{\infty}{\boldsymbol{\Theta}}}^{(\widetilde{\Gamma})}(y)
\end{aligned}
$$

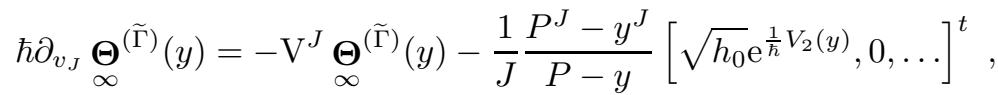

are satisfied as a consequence of the similar equations for $\underline{\Psi}_{\infty}^{(0)}(y)$ and $\underline{\Psi}_{\infty}^{(k)}(y), k=1, \ldots d_{1}$. From these, eqs. (2.2.8), (2.2.40) and (2.2.41) for $\Psi_{\infty}^{(\widetilde{\Gamma})}(x)$ follow from standard manipulations of the inverse FourierLaplace transform which are now made rigorous by the convergence of the integral. In particular this solution of (2.2.8) is associated with the function

$$
f^{(\widetilde{\Gamma})}(x):=\int_{\widetilde{\Gamma}} \mathrm{d} y \mathrm{e}^{\frac{1}{\hbar}\left(V_{2}(y)-x y\right)} .
$$


One should repeat the scheme outlined here for other contours $\widetilde{\Gamma}$ as well, until they span the same homology space spanned by the antiwedge contours $\left\{\widetilde{\Gamma}_{j}^{(y)}\right\}$, so as to obtain a basis of solutions to eq. (2.2.7). Just to complete the given example we indicate how to choose the other contours and corresponding bases:

1. The contour asymptotic to the rays $\arg (y)=-\pi$ and $\arg (y)=\pi / 10+\epsilon$. The basis on which to re-define the coefficients $\varkappa$ (which we now denote by $\tilde{\varkappa}$ ) is given by $\Gamma_{i}^{(x)} \times \Gamma_{j}^{(y)}, i \neq 6, j \neq 1$ and the wave vectors are given by

$$
\begin{gathered}
{\underset{\infty}{\boldsymbol{\Theta}}}^{\text {Left }}(y):=\frac{\Psi}{\infty}^{(0), t}(y)-2 i \pi \sum_{i=0,1,2,7} \tilde{\varkappa}^{i, 3} \frac{\Psi}{\infty}^{(i), t}(y) \\
\boldsymbol{\Theta}_{\infty}^{\text {Right }}(y):=\frac{\Psi}{\infty}^{(0), t}(y)+2 i \pi \sum_{j=3,4,5} \tilde{\varkappa}^{j, 3} \frac{\Psi}{\infty}^{(j), t}(y) .
\end{gathered}
$$

2. The contour asymptotic to the rays $\arg (y)=\pi / 2+\epsilon$ and $\arg (y)=3 \pi / 2-\epsilon$. The basis on which to re-define the coefficients $\varkappa$ is given by $\Gamma_{i}^{(x)} \times \Gamma_{j}^{(y)}, i \neq 3, j \neq 4$.

$$
\begin{gathered}
{\underset{\Theta}{\infty}}^{\text {Left }}(y):=\underline{\Psi}_{\infty}^{(0), t}(y)-2 i \pi \sum_{i=0,1,4} \tilde{\varkappa}^{i, 2} \frac{\Psi}{\infty}^{(i), t}(y) \\
{\underset{\infty}{\infty}}^{\text {Right }}(y):=\frac{\Psi}{\infty}^{(0), t}(y)+2 i \pi \sum_{j=5,6,7,0} \tilde{\varkappa}^{j, 2} \frac{\Psi}{\infty}^{(j), t}(y) .
\end{gathered}
$$

3. The contour asymptotic to the rays $\arg (y)=\pi / 2+\epsilon$ and $\arg (y)=17 \pi / 10+\epsilon$. The basis on which to re-define the coefficients $\varkappa$ is given by $\Gamma_{i}^{(x)} \times \Gamma_{j}^{(y)}, i \neq 0, j \neq 0$.

$$
\begin{gathered}
{\underset{\Theta}{\boldsymbol{\Theta}}}^{\text {Left }}(y):=\frac{\Psi}{\infty}^{(0), t}(y)-2 i \pi \sum_{i=1,2,3,4} \tilde{\varkappa}^{i, 2} \frac{\Psi}{\infty}^{(i), t}(y) \\
\boldsymbol{\Theta}^{\text {Right }}(y):=\frac{\Psi}{\infty}^{(0), t}(y)+2 i \pi \sum_{j=5,6,7} \tilde{\varkappa}^{j, 2} \frac{\Psi}{\infty}^{(j), t}(y) .
\end{gathered}
$$

We leave the details of the general case to subsequent work.

\section{References}

[1] M. Bertola, B. Eynard, J. Harnad, "An ansatz for the solution of the Riemann-Hilbert problem for biorthogonal polynomials", in preparation.

[2] M. Bertola, B. Eynard, J. Harnad, "Duality, Biorthogonal Polynomials and Multi-Matrix Models", Commun. Math. Phys., , Commun. Math. Phys. 229, 1, 73-120 (2002).

[3] M. Bertola, B. Eynard, J. Harnad, "Duality of spectral curves arising in two-matrix models" Theor. Math. Phys. (2003, in press) (NEEDS 2001 proceedings) nlin.SI/0112006. 
[4] M. Bertola, B. Eynard, J. Harnad, "Genus zero large n asymptotics of bi-orthogonal polynomials involved in the random 2-matrix model", Presentation by B.E. at AMS northeastern regional meeting, Montreal, May 3-5, 2002.

[5] M. Bertola, "Bilinear semi-classical moment functionals and their integral representation", CRM2842 (2002), math.CA/0205160.

[6] P. Bleher, A. Its, "Semiclassical asymptotics of orthogonal polynomials, Riemann-Hilbert problem, and universality in the matrix model" Ann. of Math. (2) 150, no. 1, 185-266 (1999).

[7] G. Bonnet, F. David, B. Eynard, "Breakdown of universality in multi-cut matrix models", J. Phys. A 33 6739-6768 (2000).

[8] T. S. Chihara, "An introduction to orthogonal polynomials", Mathematics and its Applications, Vol. 13 Gordon and Breach Science Publishers, New York-London-Paris, 1978.

[9] J.M. Daul, V. Kazakov, I.K. Kostov, "Rational Theories of 2D Gravity from the Two-Matrix Model", Nucl. Phys. B409, 311-338 (1993), hep-th/9303093.

[10] P. Deift, T. Kriecherbauer, K. T. R. McLaughlin, S. Venakides, Z. Zhou, "Uniform asymptotics for polynomials orthogonal with respect to varying exponential weights and applications to universality questions in random matrix theory", Commun. Pure Appl. Math. 52, 1335-1425 (1999).

[11] P. Deift, T. Kriecherbauer, K. T. R. McLaughlin, S. Venakides, Z. Zhou, "Strong asymptotics of orthogonal polynomials with respect to exponential weights", Commun. Pure Appl. Math. 52, 1491$1552,(1999)$.

[12] P. Di Francesco, P. Ginsparg, J. Zinn-Justin, "2D Gravity and Random Matrices", Phys. Rep. 254, 1 (1995).

[13] N. M. Ercolani and K. T.-R. McLaughlin "Asymptotics and integrable structures for biorthogonal polynomials associated to a random two-matrix model", Physica D, 152-153, 232-268 (2001).

[14] B. Eynard, "Eigenvalue distribution of large random matrices, from one matrix to several coupled matrices" Nucl. Phys. B 506, 633 (1997), cond-mat/9707005.

[15] B. Eynard, "Correlation functions of eigenvalues of multi-matrix models, and the limit of a time dependent matrix", J. Phys. A: Math. Gen. 31, 8081 (1998), cond-mat/9801075.

[16] B. Eynard, M.L. Mehta, "Matrices coupled in a chain: eigenvalue correlations", J. Phys. A: Math. Gen. 31, 4449 (1998), cond-mat/9710230. 
[17] A. Fokas, A. Its, A. Kitaev, "The isomonodromy approach to matrix models in 2D quantum gravity", Commun. Math. Phys. 147, 395-430 (1992).

[18] A. Guionnet, Zeitouni, "Large deviations asymptotics for spherical integrals", J. F. A. 188, 461-515 (2002).

[19] A. R. Its, A. V. Kitaev, A. S. Fokas "Matrix models of two-dimensional quantum gravity and isomonodromy solutions of "discrete Painleve equations" ",Zap. Nauch. Sem. LOMI, 187, 3-30 (1991) (Russian), translation in J. Math. Sci. 73, no. 4, 415-429 (1995).

[20] A.R. Its, A.V. Kitaev, and A.S. Fokas, "An isomonodromic Approach in the Theory of TwoDimensional Quantum Gravity", Usp. Matem. Nauk, 45, 6 (276 ), 135-136 (1990), (Russian), translation in Russian Math. Surveys, 45, no. 6, 155-157 (1990).

[21] A. A. Kapaev, "The Riemann-Hilbert problem for the bi-orthogonal polynomials", nlin.SI/0207036.

[22] V.A. Kazakov, "Ising model on a dynamical planar random lattice: exact solution", Phys Lett. A119, 140-144 (1986).

[23] A. Matytsin, "on the large $N$ limit of the Itzykson Zuber Integral", Nuc. Phys. B411, 805 (1994), hep-th/9306077.

[24] M.L. Mehta, "Random Matrices, second edition", Academic Press, New York, 1991.

[25] G. Szegö "Orthogonal Polynomials", AMS, Providence, Rhode Island, (1939).

[26] K. Ueno and K. Takasaki, "Toda Lattice Hierarchy", Adv. Studies Pure Math. 4, 1-95 (1984). 